\title{
Equilibrium Melting Temperature Depression in Syndiotactic Poly(styrene-stat-3- methylstyrene) and Poly(styrene-stat-4-methylstyrene)
}

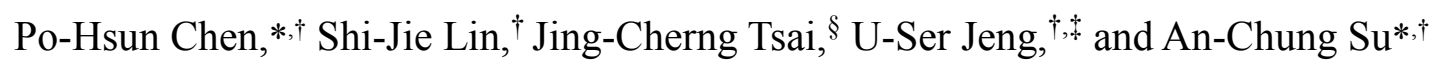
†Department of Chemical Engineering, National Tsing Hua University, Hsinchu 30013, Taiwan ${ }^{\S}$ Department of Chemical Engineering, National Chung Cheng University, Chiayi 62102, Taiwan *National Synchrotron Radiation Research Center, Science Park, Hsinchu, 30076, Taiwan

*E-mail: zero00023@gapp.nthu.edu.tw (Po-Hsun Chen); acsu@mx.nthu.edu.tw (An-Chung Su)

\section{Content}

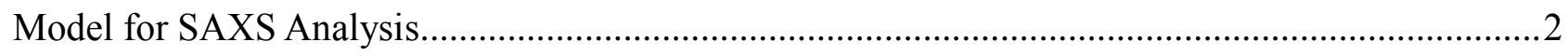

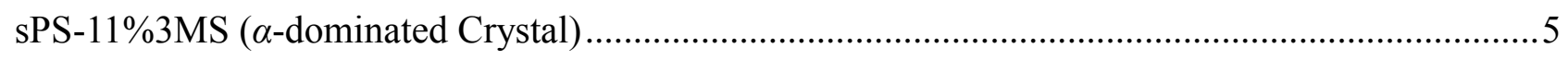

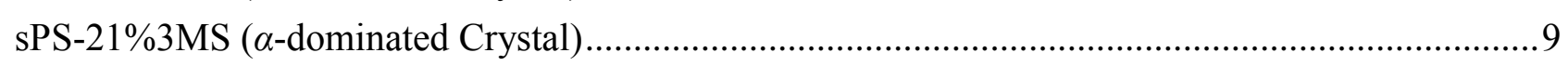

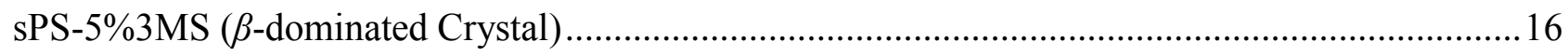

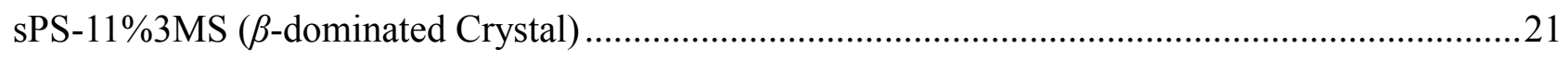

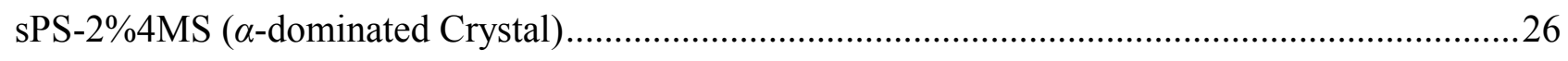

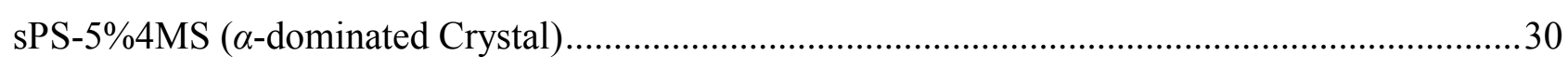

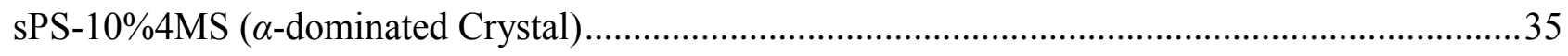

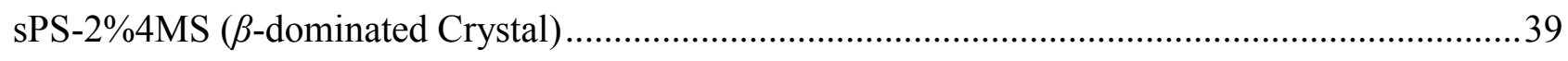

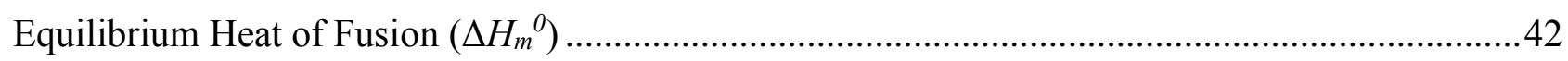

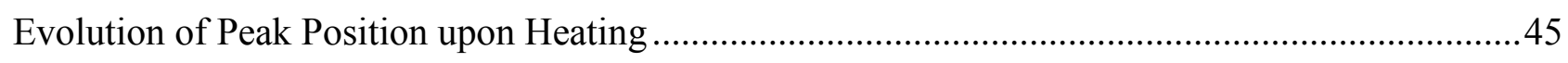

$\alpha$ - and $\beta$-structure (Molecular Mechanics Computation) ..........................................................46

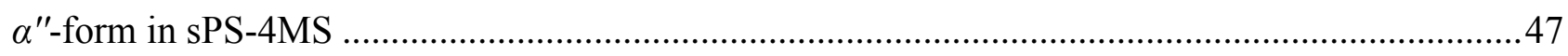

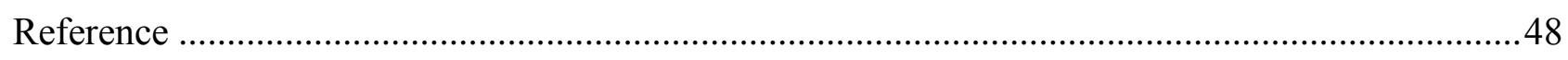




\section{Model for SAXS Analysis}

In our previous report, which determines the thermodynamic parameters of the sPS homopolymer $\alpha$ - and $\beta$-phase, the method for extract thickness information from the SAXS profiles via 1-D correlation function and Kratky-Porod approximation are compared. ${ }^{1} 1$-D correlation function is a conventional method to determines the thickness of scattering object by assuming an ideal lamellar morphology. The results from the correlation function and Kratky-Porod approximation agree well at lower temperatures ( $c f$. Figures 2 and 5 in Reference 1). However, the correlation function starts to distort as the temperature increase, indicating that the spatial arrangement of the crystal deviates from the well-behaved lamellar structure (Figure S1 and Figures 2 and Figure 5 in Reference 1). In contrast, the Kratky-Porod approximation is shown to be more applicable at a higher temperature region. The crystal thickness can be extracted from the slope of the Kratky-Porod plot ( $c f$. Figures 2 and 5 in Reference 1), but the slope determination is more or less a subjective process. By modeling the SAXS profiles with $I(q)=I_{0} P(q) S(q)\left(I_{0}\right.$ is the zeroangle scattering intensity, $P(q)$ is the normalized form factor, and $S(q)$ the structure factor of the scattering particles), the dimension of nanograins can be determined by the form factor model fitting.

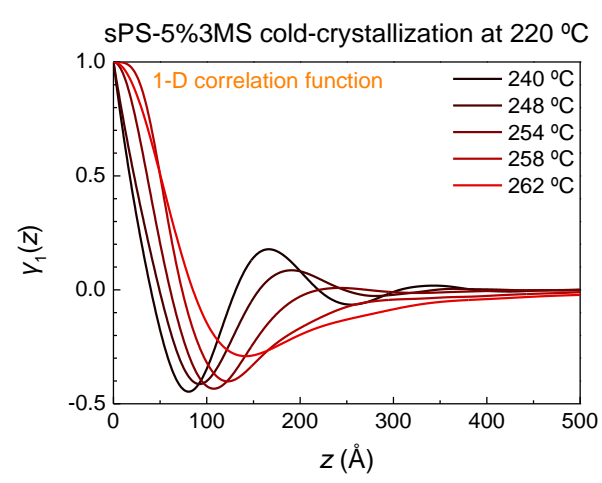

Figure S1. Representative 1-D correlation function for sPS-5\%3MS cold-crystallized specimen $\left(T_{c}\right.$ $=220^{\circ} \mathrm{C}$ ) upon heating. At lower temperatures, well-behaved correlation profiles can be obtained; however, as the temperature increase, the correlation profiles start to be distorted and the crystal long period cannot be defined clearly. 
Illustrated in Figure S2 are the SAXS profiles of sPS-5\%3MS specimen (cold-crystallization, $T_{c}=220^{\circ} \mathrm{C}$ ) at $261{ }^{\circ} \mathrm{C}$ (dot symbol) that the contribution of amorphous molten state has been subtracted, and the red solid lines are the fitting profiles with different shape of form factor, containing (a) sphere, (b) ellipsoid, (c) cylinder, (d) cylinder with polydisperse length and (e) the cylinder with polydisperse radius. Except for the ellipsoid form factor, which was used TableCurve 2D software, Igor software was used to fit with sphere and cylinder series form factors. Among these different shapes of the form factor, the cylinder with polydisperse radius gives the best fit.

(a)

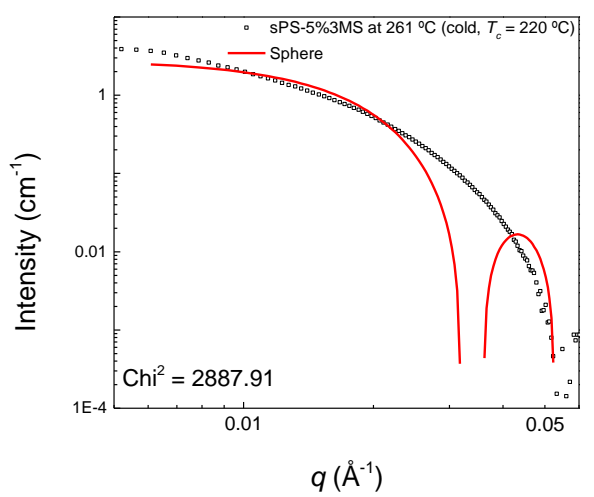

(c)

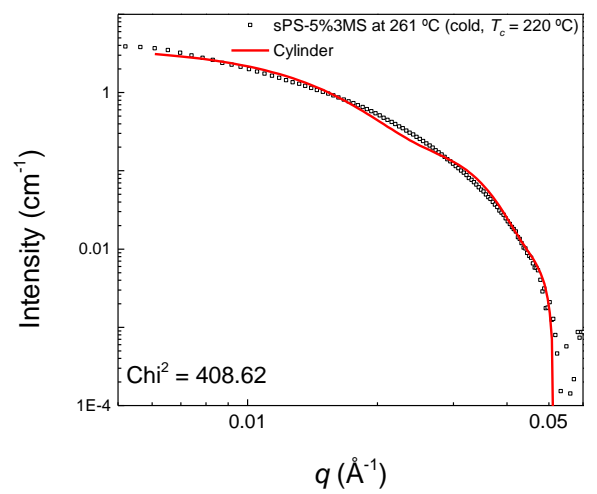

(e) Fitting Model: Cylinder (Polyradius)

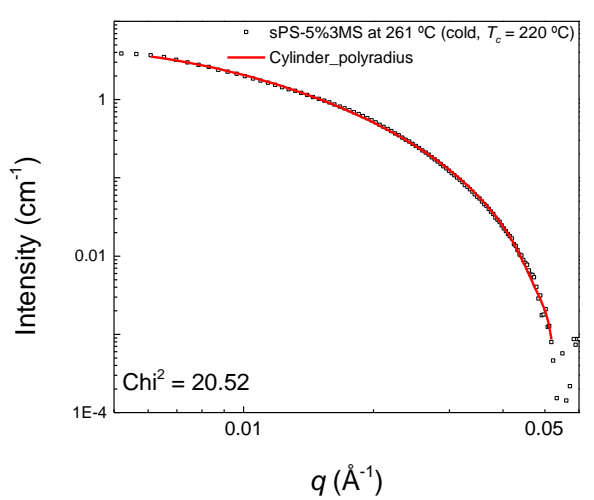

(b) Fitting Model: Ellipsoid

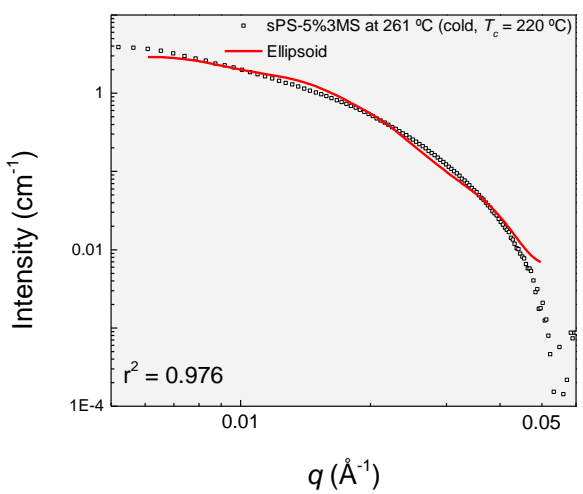

(d) Fitting Model: Cylinder (Polylength)

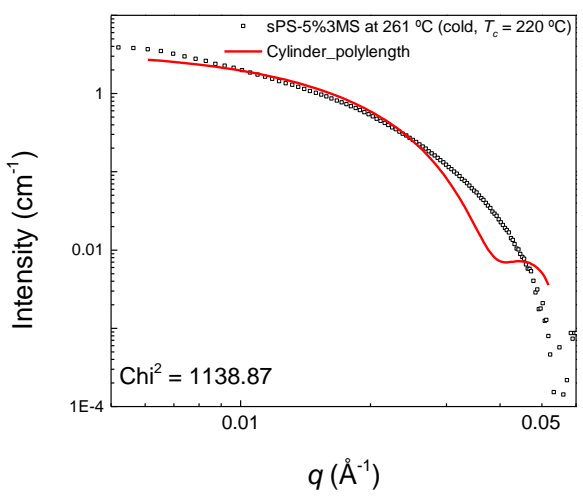

Figure S2. Experimental SAXS profile of sPS-5\%3MS cold-crystallized $\left(T_{c}=220^{\circ} \mathrm{C}\right)$ specimen at $261{ }^{\circ} \mathrm{C}$ (dot symbol) with the fitting results of different form model (red line), including (a) sphere, (b) ellipsoid, (c) cylinder, (d) cylinder with disperse length, and (e) cylinder with disperse radius. Among these models, the cylinder with disperse radius model gives the best fit. 
Additionally, the Kratky-Porod approximation for the slablike scattering object is $I(q) \propto q^{-2}$ $\exp \left(-q^{2} l_{c}^{2} / 12\right)$ and the representative Kratky-Porod plot, i.e., $\ln \left[I(q) q^{2}\right]$ vs. $q^{2}$, for sPS-5\%3MS coldcrystallization at $220^{\circ} \mathrm{C}$ specimen is illustrated in Figure S3a. The thickness of the slablike scattering object can be determined from the slop $\left(=l_{c}^{2} / 12\right)$ of the Kratky-Porod plot, which indicates by the solid line in Figure S3a. Figure S3b represents the reciprocal crystal thickness evolution $\left(1 / l_{c}\right)$ against the temperature upon heating for sPS-5\%3MS $\alpha$-crystal. The equilibrium melting temperature $\left(T_{m}{ }^{0}\right)$ can be determined via the constructed melting line which indicates as dash line in the figure. The $T_{m}{ }^{0}$ determined from the Kratky-Porod approximation $\left(283.3 \pm 3.5^{\circ} \mathrm{C}\right)$ for $\mathrm{sPS}-5 \% 3 \mathrm{MS} \alpha$-crystal is comparable to the value determined from the form factor model fitting method as described in the main article ( $c f$. Figure 5). Based on the above discussion, the form factor model of cylinder shape with polydisperse radius has been used to fit the SAXS profiles in this article.
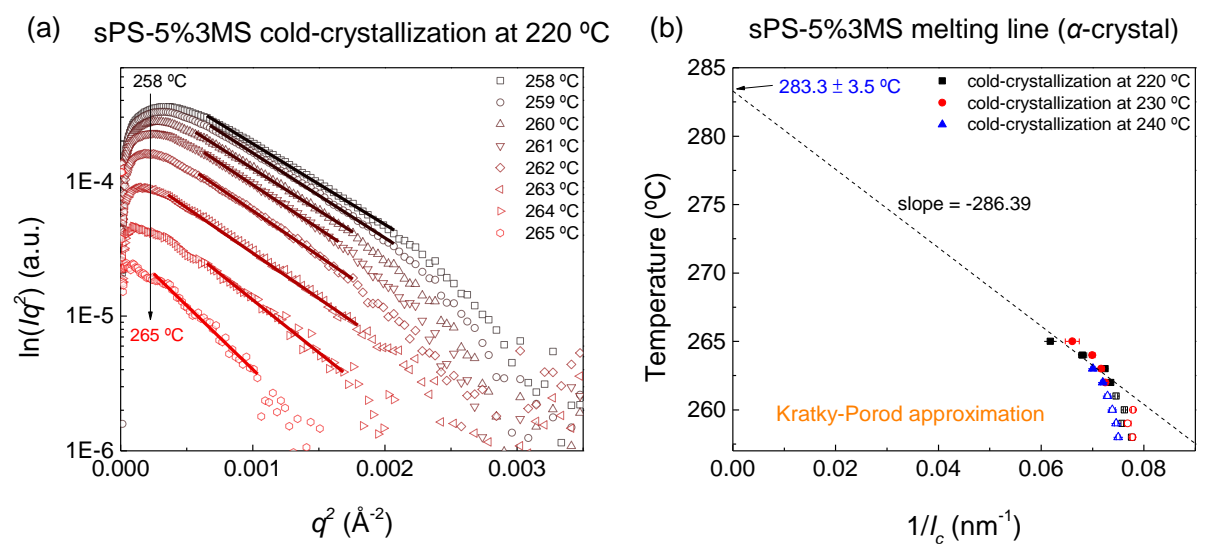

Figure S3. (a) Representative Kratky-Porod plot for sPS-5\%3MS cold-crystallization at $220^{\circ} \mathrm{C}$ upon heating. Solid lines represent the linear region to determine the thickness of the slablike scattering object. (b) Evolution of the reciprocal crystal thickness $\left(1 / l_{c}\right.$, determining from KratkyPorod approximation) of sPS-5\%3MS cold-crystallized specimens upon heating. Dash line represents the melting line that extrapolates by the data points which are higher than $262{ }^{\circ} \mathrm{C}$ (solid symbols), giving the equilibrium melting temperature as $283.3 \pm 3.5^{\circ} \mathrm{C}$. 
The simultaneous SAXS/WAXS heating profiles of sPS-11\%3MS cold-crystallized specimens are illustrated in Figure S4. Compared among each initial scattering profile (black solid lines), increasing $T_{c}$ makes the lamellar peak position $\left(q_{c}\right)$ shifts to lower $q$-value, implying that higher isothermal temperature gives larger long period (inter-particle distance). Corresponding WAXS profiles in Figure S4c-d show that specimens were all formed $\alpha$-dominated crystal. Upon heating, the lamellar peak in SAXS profiles progressively become broader and $q_{c}$ shifts to lower $q$ position, which represents the increase of long-period and weakening of the spatial correlation between nanograins; in WAXS profiles, the reflection peaks gradually decrease without appearing any new reflection peak. Thus, the SAXS/WAXS profiles correspond to the melting behaviors of cold-crystallized sPS- $11 \% 3 \mathrm{MS}$ specimens. Besides, after completely melt at ca. $256{ }^{\circ} \mathrm{C}$, the SAXS profiles (red sloid lines in Figure $\mathrm{S} 4 \mathrm{a}-\mathrm{b}$ ) give the power-law decrease scattering with exponent ca. -3 and only show amorphous profiles in the WAXS profiles.

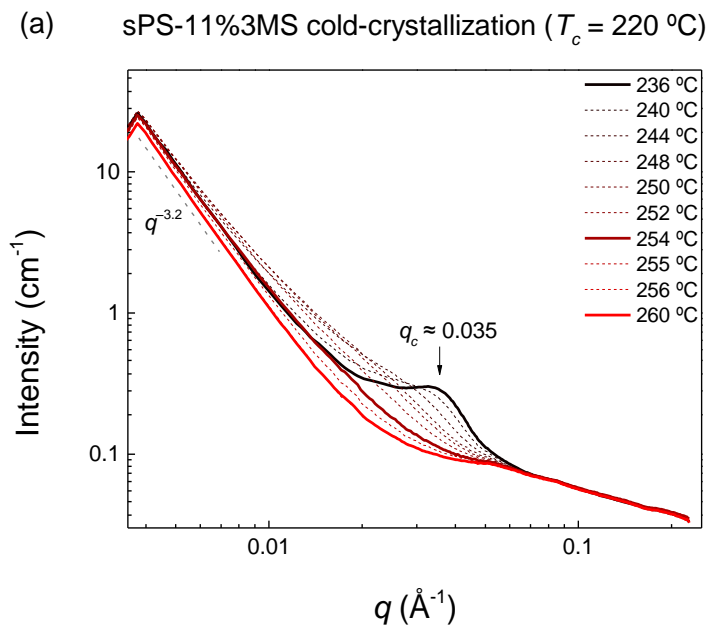

(b) SPS-11\%3MS cold-crystallization $\left(T_{c}=240^{\circ} \mathrm{C}\right)$

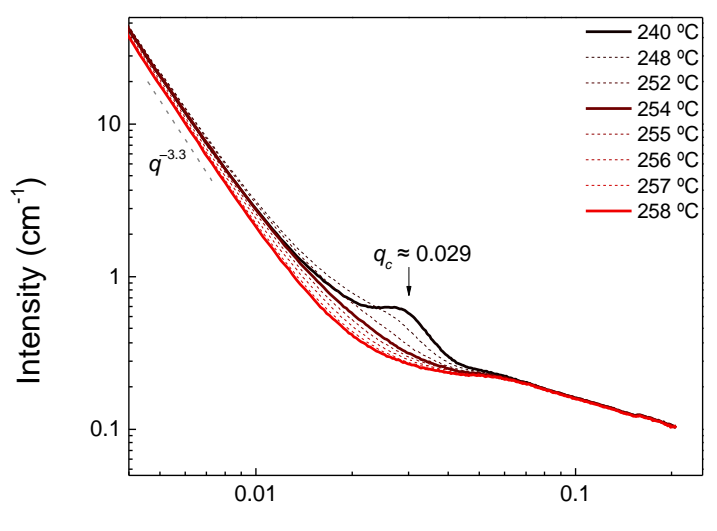

$q\left(\AA^{-1}\right)$ (c) sPS-11\%3MS cold-crystallization $\left(T_{c}=220^{\circ} \mathrm{C}\right)$

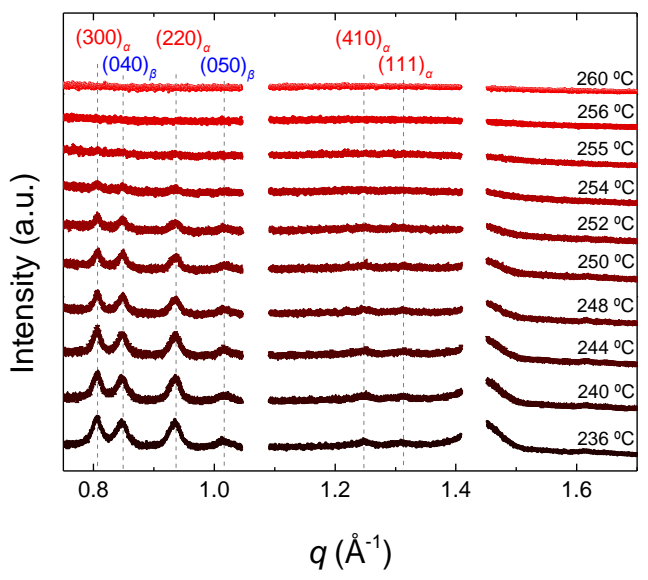

(d) sPS-11\%3MS cold-crystallization $\left(T_{c}=240^{\circ} \mathrm{C}\right)$

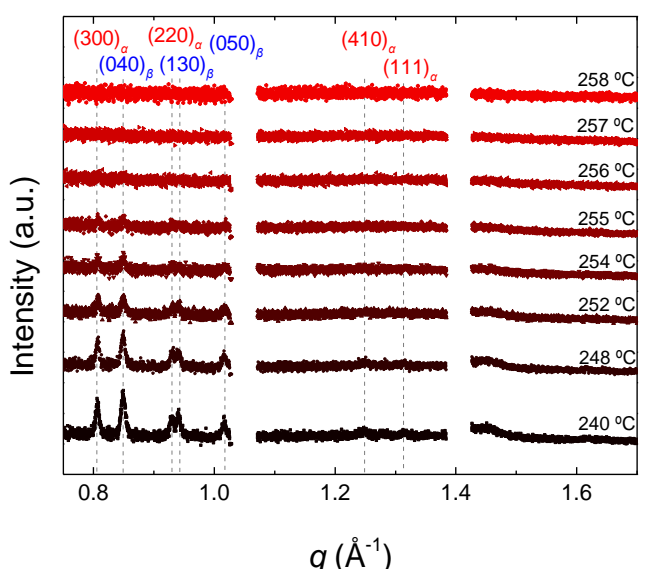

Figure S4. Simultaneous (a)-(b) SAXS and (c)-(d) WAXS heating profiles of sPS-11\%3MS coldcrystallized specimens at rate $2{ }^{\circ} \mathrm{C} / \mathrm{min}$. Black solid lines in the SAXS profiles represent at lower temperatures, and the red solid lines denote the profiles of the molten state; dash lines in the low $q$ - 
region indicate the slope of the power-law decrease for molten state scattering, and all give the value ca. 3. The WAXS profiles show that all give $\alpha$-dominated crystals, and the reflection peaks decrease gradually upon heating and completely melt after reaching ca. $256^{\circ} \mathrm{C}$ without emerging new peaks.

Illustrated in Figure S5a-b are the schematic peak deconvoluted WAXS profiles, and relative peak area presented in $\mathrm{c}-\mathrm{d}$ was calculated based on the deconvoluted results with normalizing to initial value respectively. Depending on the evolution of relative peak area, the $\alpha$-crystals always have higher population than $\beta$-crystals and populations of both $\alpha$ - and $\beta$-crystals decrease progressively upon heating. Thus, the melting process dominates the heating scan.

(a) $\mathrm{sPS}-11 \% 3 \mathrm{MS}$ (cold-crystallized, $T_{c}=220^{\circ} \mathrm{C}$ )

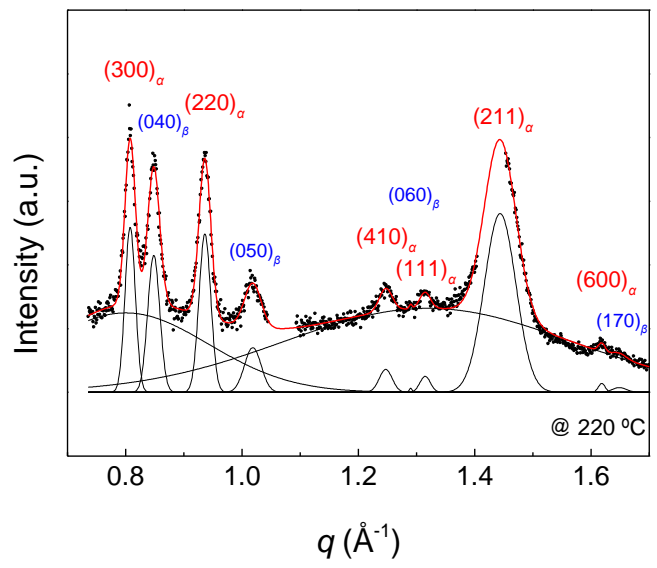

(b) sPS-11\%3MS (cold-crystallized, $T_{c}=240 \stackrel{\circ}{\circ}$ )

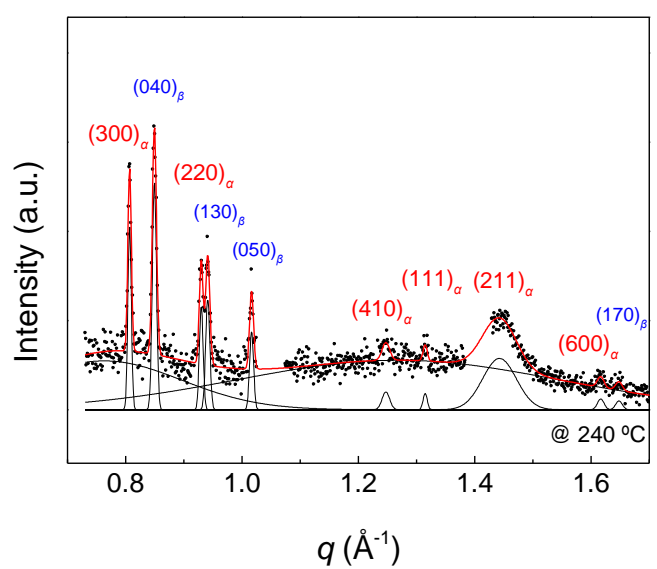

(c) SPS-11\%3MS (cold-crystallized, $T_{c}=220 \stackrel{\circ}{\circ}$ )

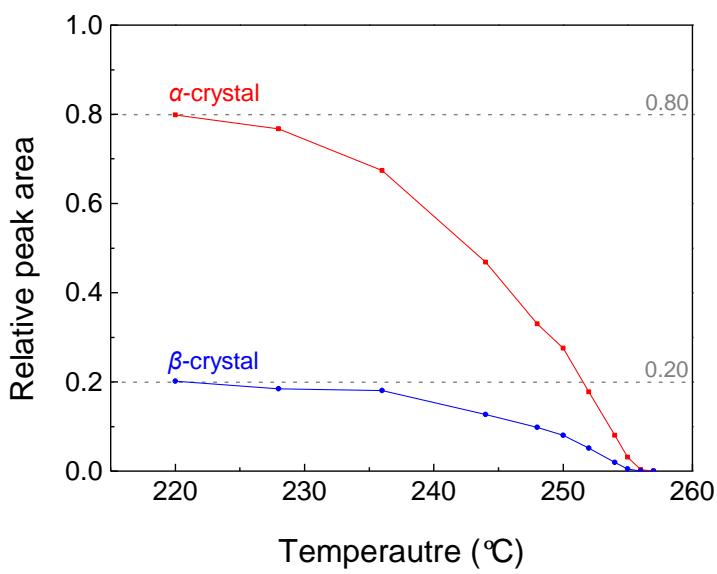

(d) SPS-11\%3MS (cold-crystallized, $T_{c}=240 \stackrel{\circ}{\circ}$ )

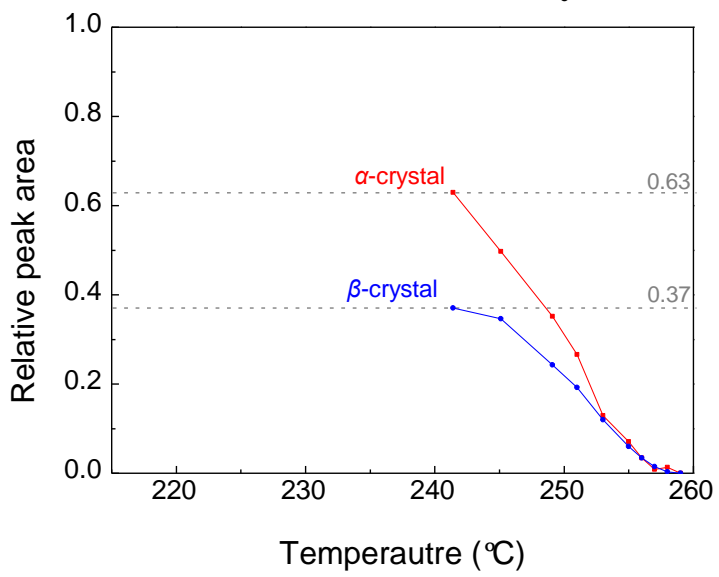

Figure S5. The schematic peak deconvolution WAXS profiles of sPS-11\%3MS cold-crystallized at (a) 220 and (b) $240{ }^{\circ} \mathrm{C}$, indicating that the diffraction profiles consist of two amorphous halos and reflection peaks of $\alpha$ - and $\beta$-crystals. The relatively crystalline peak area between $\alpha$ - and $\beta$-crystals were given in (c) $-(d)$ that the values were normalized with the initial values respectively, which denotes that the $\alpha$-crystals dominate the crystalline population upon heating.

After subtracting the molten state scattering profiles, the reduced sPS-11\%3MS coldcrystallized SAXS heating profiles that approached melting are shown in Figure S6. Open symbols are the experimental data, and the solid lines are the fitting result by the use of the cylinder-shaped form factor with disperse radius. The corresponding fitting parameters of the radius and thickness 
are presented in Figure S7, and the relation between reciprocal crystal thickness $\left(l_{c}{ }^{-1}\right)$ and the experimental temperature is illustrated in Figure S8. The melting line can be constructed by extrapolating the data points (solid symbol), which is just several degrees below melting, and for the case of $l_{c}{ }^{-1} \sim 0$ gives the equilibrium melting temperature $\left(T_{m}{ }^{0}\right)$ of sPS-11\%3MS $\alpha$-crystal as $266.2 \pm 5.4^{\circ} \mathrm{C}$. Based on the depression level of $T_{m}{ }^{0}$ and the slope of the melting line, the penalty energy $(\varepsilon)$ and the fold surface energy $\left(\sigma_{e}\right)$ can be derived via Sanchez-Eby and Gibbs-Thomson theory, which is $\varepsilon=41.0 \pm 17.4 \mathrm{MJ} \mathrm{m}^{-3}$ and $\sigma_{e}=11.0 \pm 5.4 \mathrm{~mJ} \mathrm{~m}^{-2}$.

(a) sPS-11\%3MS (cold-crystallized, $T_{c}=220 \stackrel{\circ}{ } \mathrm{C}$ )

(b) sPS-11\%3MS (cold-crystallized, $T_{c}=240^{\circ} \mathrm{C}$ )
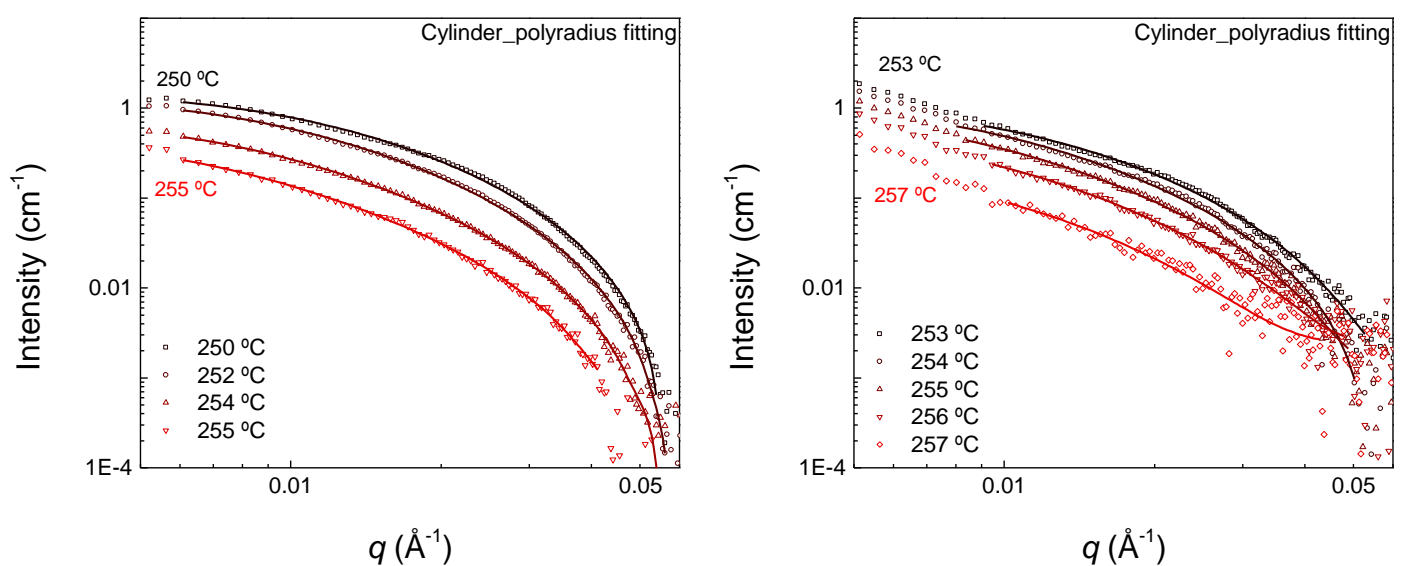

Figure S6. Reduced SAXS heating profiles of sPS-11\%3MS cold-crystallized at $T_{c}=$ (a) 220 and (b) $240{ }^{\circ} \mathrm{C}$ before melting (ca. 250 to $257^{\circ} \mathrm{C}$ ), which gives basically form factor scattering. The solid lines represent the fitting profiles by using the cylinder-shaped form factor with polydisperse radius.

(a) $\quad$ sPS-11\%3MS fitting result (radius)

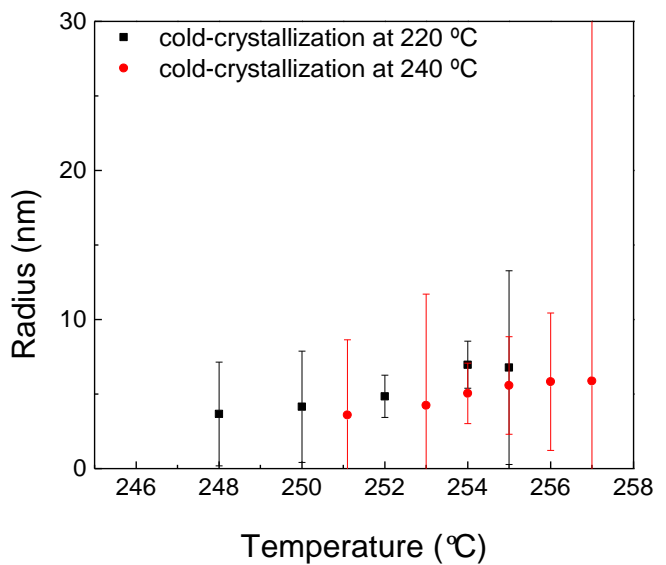

(b) sPS-11\%3MS fitting result (thickness)

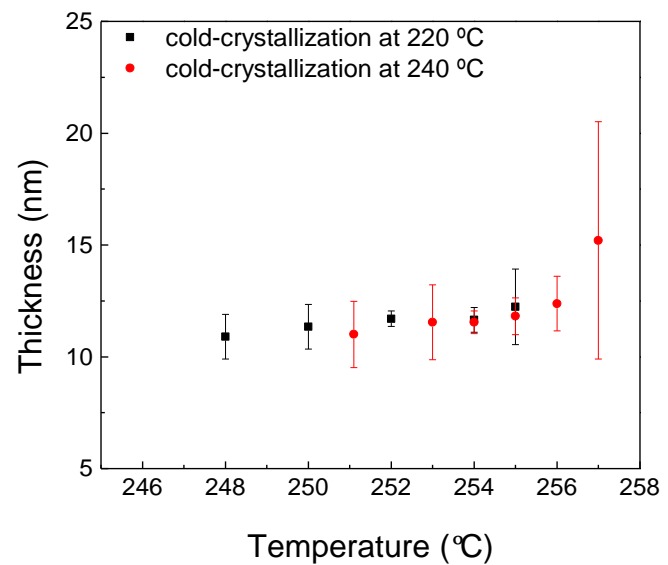

Figure S7. Evolution of (a) radius and (b) thickness of the cylinder-shaped form factor parameters upon heating, which were obtained from the reduced SAXS profiles in Figure S6. Depending on the fitting results, the nanograins are close to a disk-like shape. 


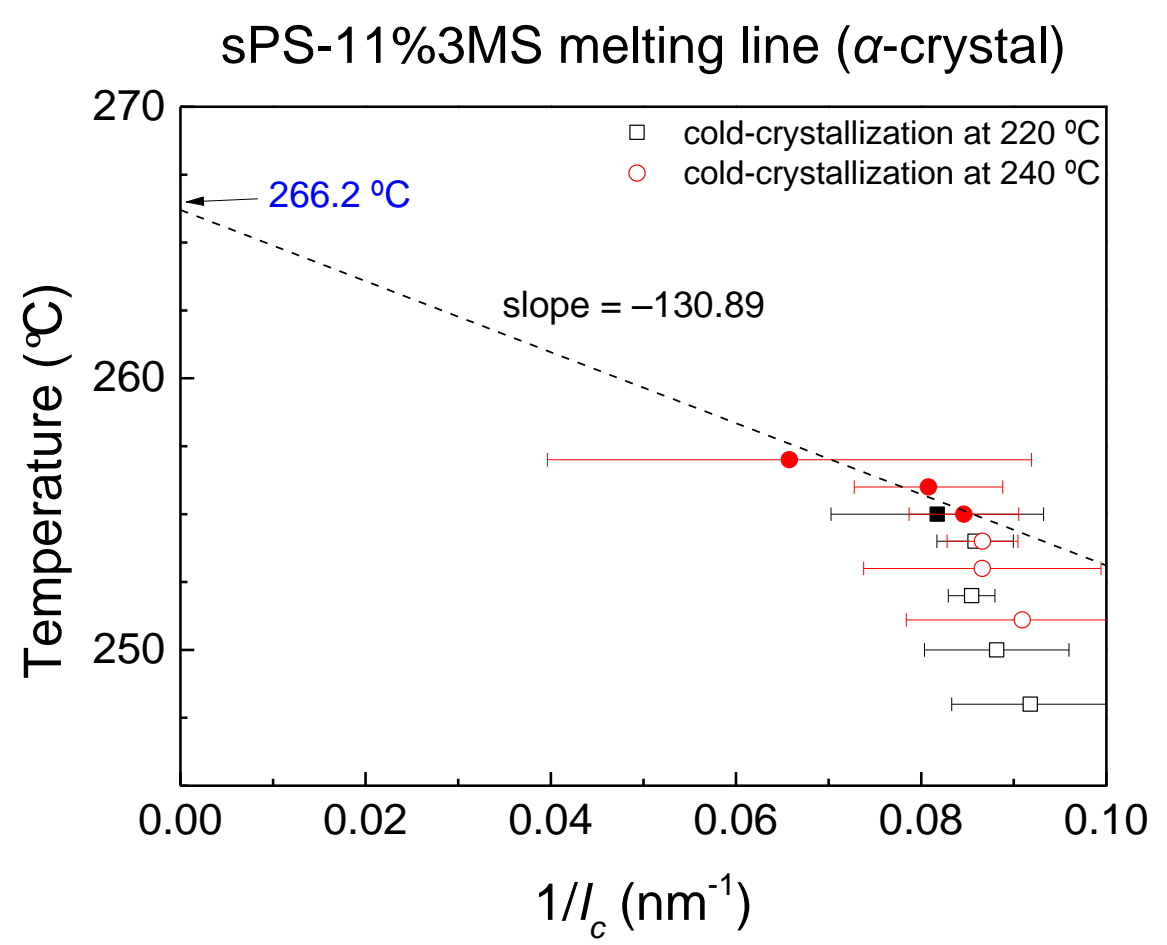

Figure S8. Evolution of the reciprocal crystal thickness $\left(1 / l_{c}\right)$ upon heating for sPS-11\%3MS coldcrystallized specimens. Dash line represents the melting line that established by extrapolating the data points which were higher than $255^{\circ} \mathrm{C}$ (solid symbols), giving the equilibrium melting temperature $\left(T_{m}{ }^{0}\right)$ for sPS-11\%3MS cold-crystallized $\alpha$-crystals as $266.2 \pm 5.4{ }^{\circ} \mathrm{C}$. 
The simultaneous SAXS/WAXS heating profiles of sPS-21\%3MS cold- and melt-crystallized specimens are illustrated in Figure S9-10. Depending on the WAXS profiles, both cold- and meltcrystallized specimens were all formed $\alpha$-dominated crystal. Compared among each initial scattering in the SAXS profiles (black solid lines), specimen with higher $T_{c}$ has lower lamellar peak position $\left(q_{c}\right)$, implying that higher isothermal temperature gives larger long period (inter-particle distance). Upon heating, the lamellar peak in SAXS profiles progressively become broader and $q_{c}$ shifts to lower $q$-position, which represents the increase of long-period and weakening of the spatial correlation between nanograins; in WAXS profiles, the reflection peaks gradually decrease without appearing any new reflection peak. Thus, the SAXS/WAXS profiles correspond to the melting behaviors of sPS-21\%3MS specimens. Besides, after completely melt at ca. $257^{\circ} \mathrm{C}$, the SAXS profiles (red sloid lines in Figure $\mathrm{S} 9 \mathrm{a}-\mathrm{c}$ and $\mathrm{S} 10 \mathrm{a}-\mathrm{b}$ ) give the power-law decrease scattering with exponent ca. -3 and only show amorphous profiles in the WAXS results. 
(a) sPS-21\%3MS cold-crystallization $\left(T_{c}=220 \stackrel{\circ}{ } \mathrm{C}\right)$

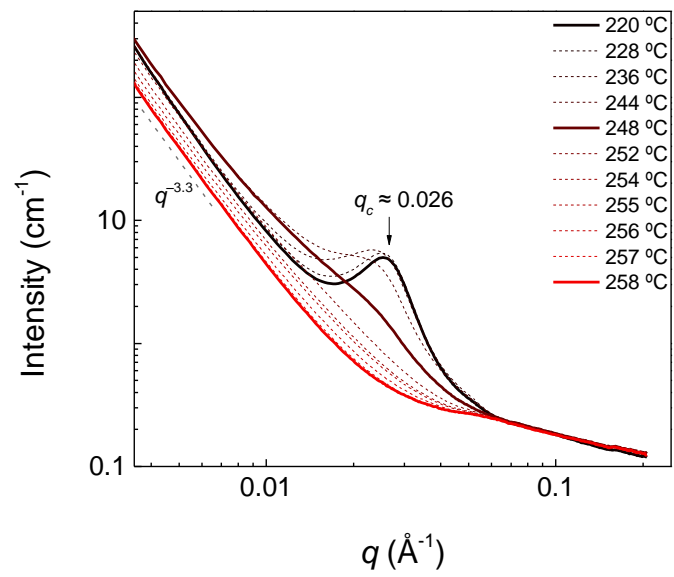

(b) $\quad$ PPS-21\%3MS cold-crystallization $\left(T_{c}=230^{\circ} \mathrm{C}\right)$

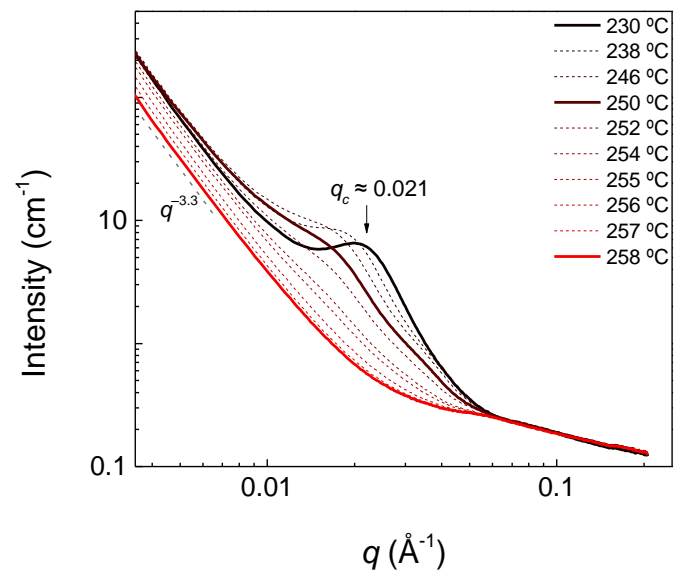

(c) $\mathrm{sPS}-21 \% 3 \mathrm{MS}$ cold-crystallization $\left(T_{c}=240^{\circ} \mathrm{C}\right)$

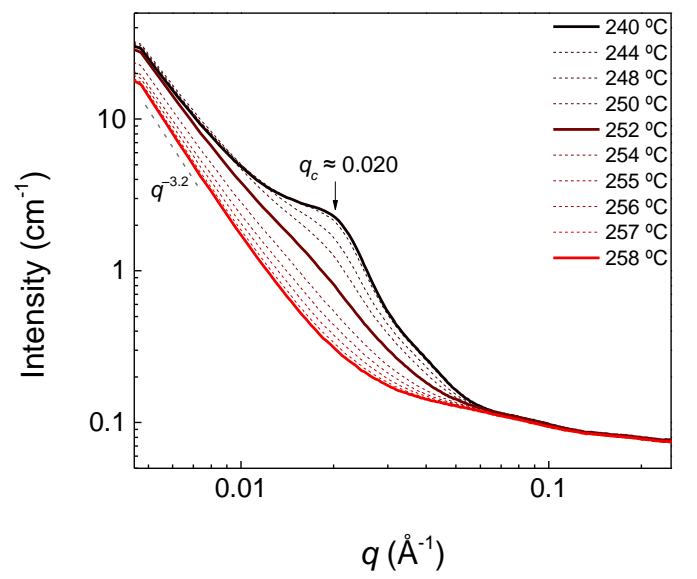

(d)

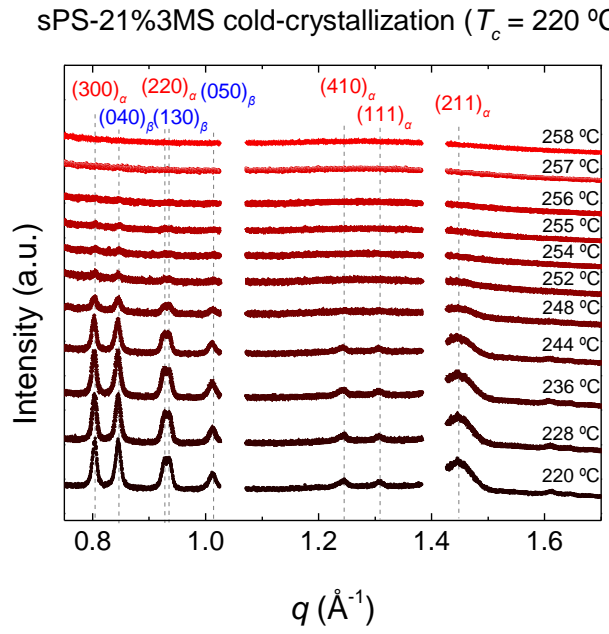

(e) $\quad$ sPS-21\%3MS cold-crystallization $\left(T_{c}=230 \stackrel{\circ}{\circ}\right)$

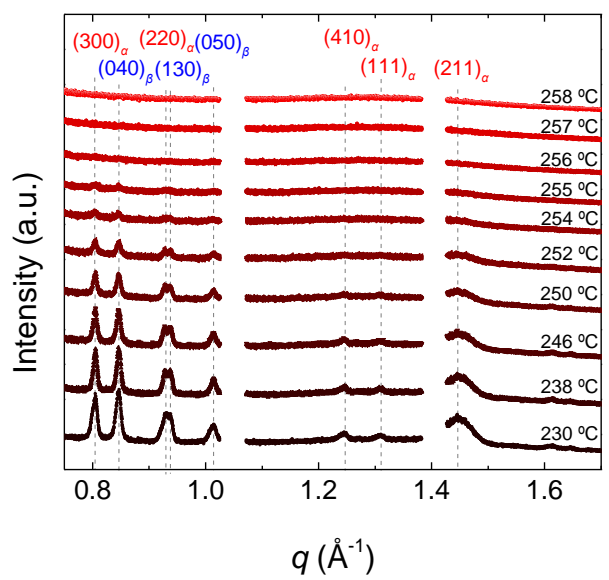

(f) $\quad$ SPS-21\%3MS cold-crystallization $\left(T_{c}=240^{\circ} \mathrm{C}\right)$

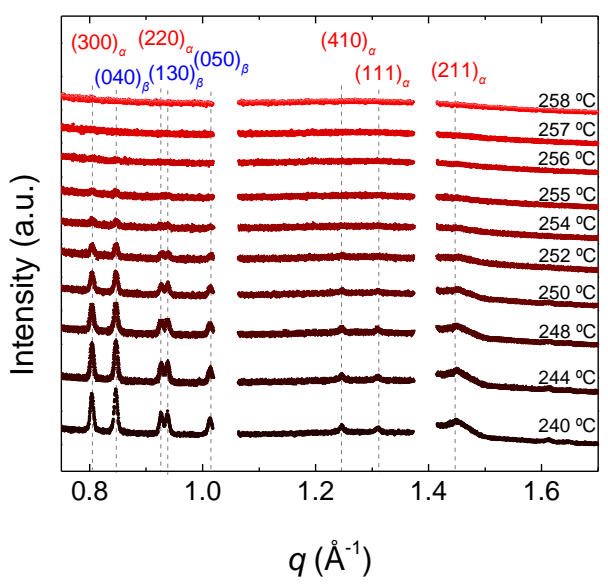

Figure S9. Simultaneous (a)-(c) SAXS and (d)-(f) WAXS heating profiles of sPS-21\%3MS coldcrystallized specimens from each $T_{c}$ to the molten state. Black solid lines in the SAXS profiles represent the starting profiles (at $T_{c}$ ) for each specimen, and the red solid lines correspond to the scattering of molten state. The WAXS profiles show that the reflections peaks gradually decrease, and the $\alpha$-dominated crystals completely melt after reaching ca. $258^{\circ} \mathrm{C}$, which gives only the amorphous halo. 
(a) $\quad$ sPS-21\%3MS melt-crystallization $\left(T_{c}=220^{\circ} \mathrm{C}\right)$

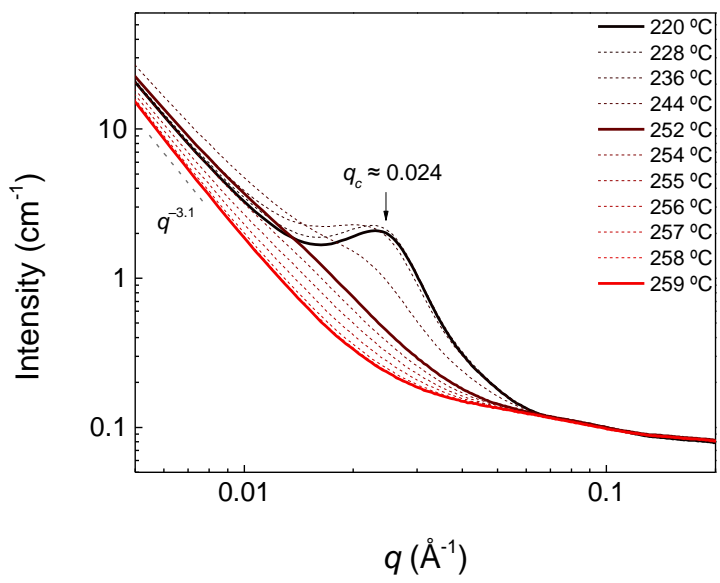

(b) $\quad$ sPS-21\%3MS melt-crystallization $\left(T_{c}=235^{\circ} \mathrm{C}\right)$

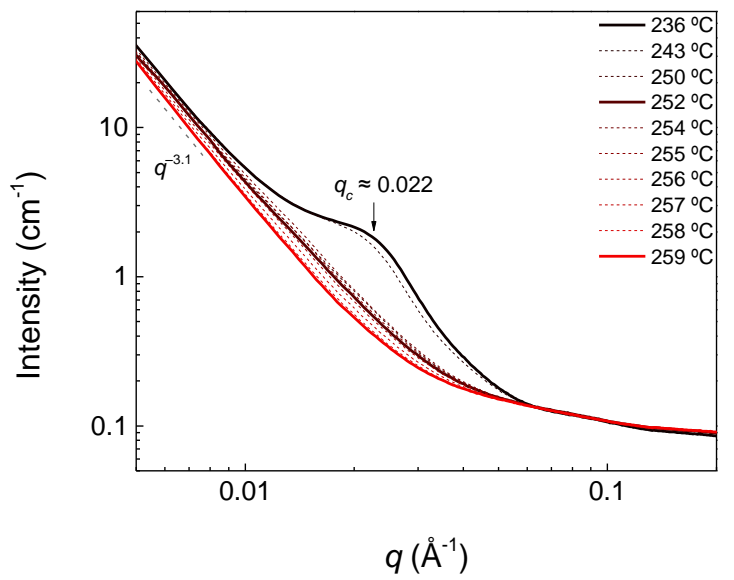

(c) sPS-21\%3MS melt-crystallization $\left(T_{c}=220^{\circ} \mathrm{C}\right)$

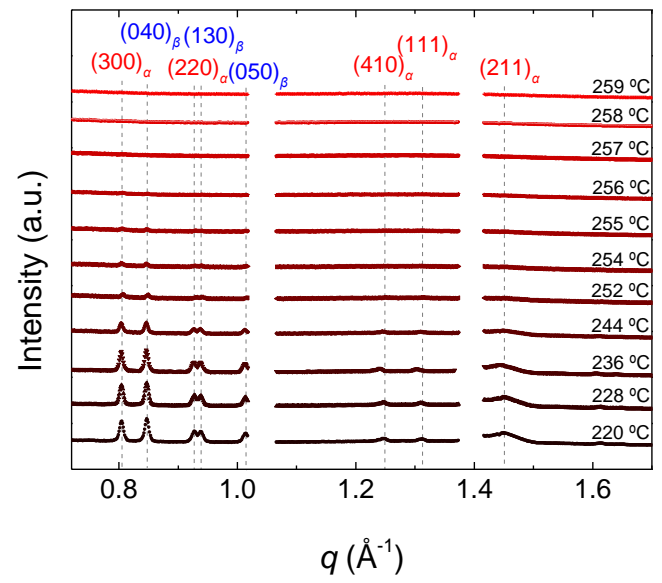

(d) $\mathrm{sPS}-21 \% 3 \mathrm{MS}$ melt-crystallization $\left(T_{c}=235^{\circ} \mathrm{C}\right)$

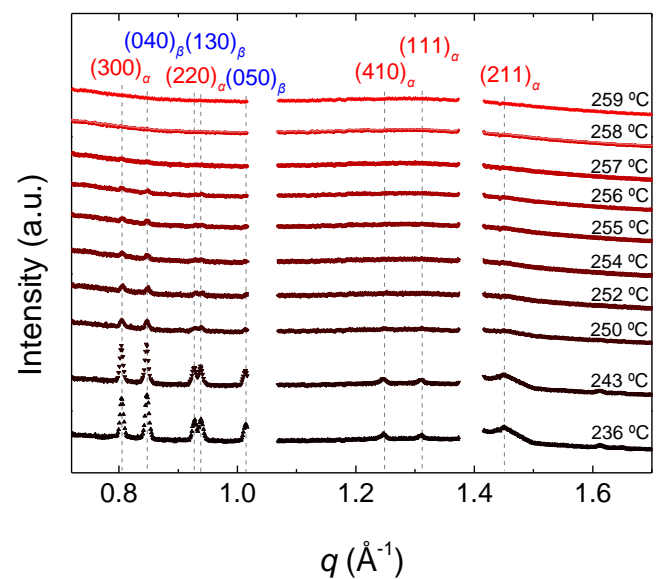

Figure S10. Simultaneous (a)-(b) SAXS and (c)-(d) WAXS heating profiles of sPS-21\%3MS meltcrystallized specimens from each $T_{c}$ to molten state. Black solid lines in the SAXS profiles represent the starting profiles (at $T_{c}$ ) for each specimen, and the red solid lines are the scattering profiles of molten state. The corresponding WAXS profiles show that the diffraction peaks gradually decrease, and the $\alpha$-dominated crystals completely melt after reaching ca. $257^{\circ} \mathrm{C}$ which gives amorphous profiles.

Illustrated in Figure S11a-c and S12a-b are the schematic peak deconvoluted WAXS profiles, and the relative peak area presented Figure S11d-f and S12c-d were calculated based on the deconvoluted results with normalizing to initial value (at $T_{c}$ ) respectively. Depending on the evolution of relative peak area, the $\alpha$-crystals always have higher population than $\beta$-crystals and populations of both $\alpha$ - and $\beta$-crystals decrease progressively upon heating. Thus, the melting process dominates upon heating. 
(a) sPS-21\%3MS (cold-crystallized, $T_{c}=220^{\circ} \mathrm{C}$ )

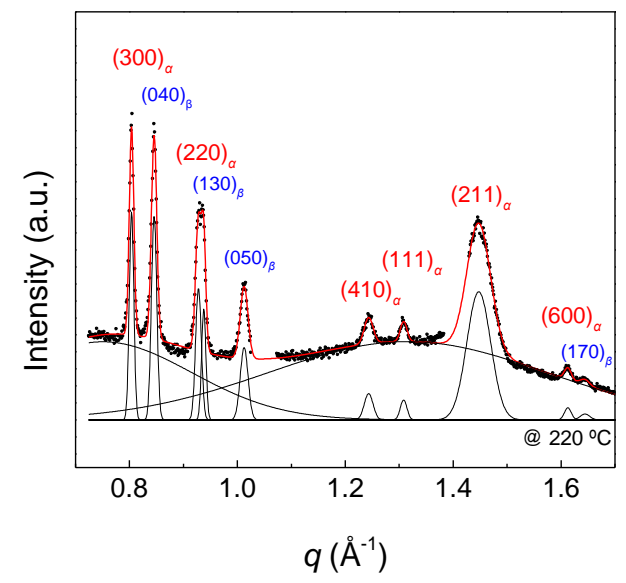

(b) sPS-21\%3MS (cold-crystallized, $T_{c}=230^{\circ} \mathrm{C}$ )

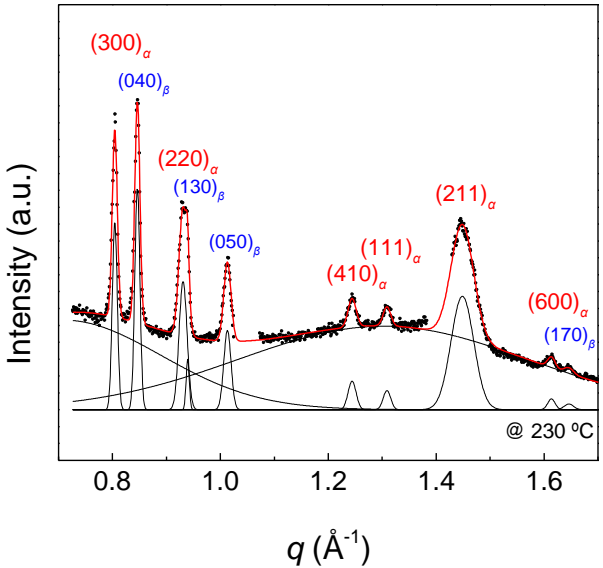

(c) SPS-21\%3MS (cold-crystallized, $T_{c}=240 \stackrel{\circ}{\circ}$ )

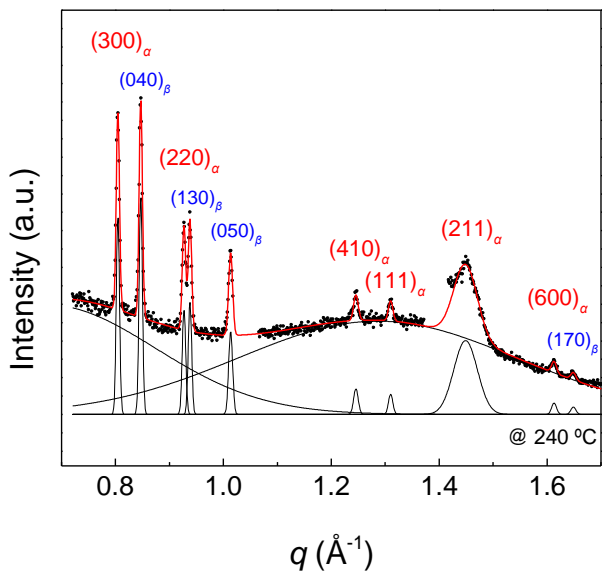

(d) sPS-21\%3MS (cold-crystallized, $T_{c}=220{ }^{\circ} \mathrm{C}$ )

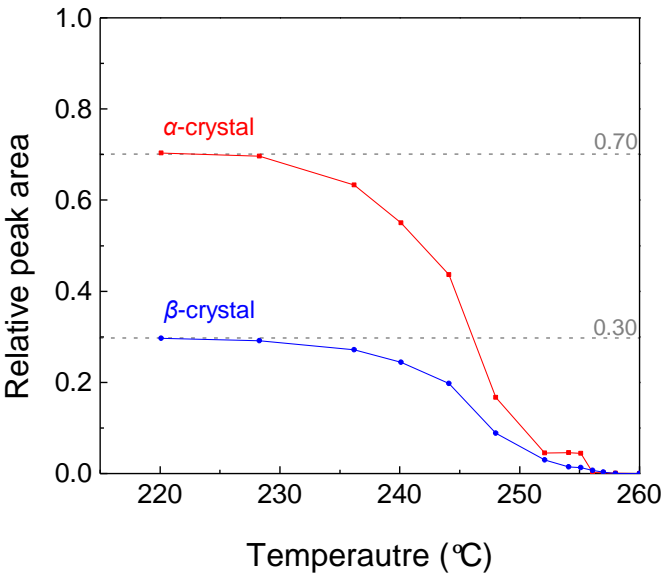

(e) $\quad$ sPS-21\%3MS (cold-crystallized, $T_{c}=230^{\circ} \mathrm{C}$ )

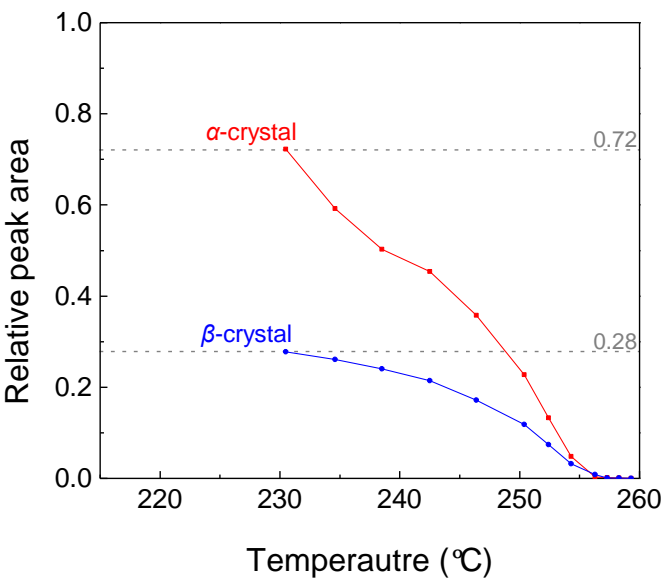

(f) sPS-21\%3MS (cold-crystallized, $T_{c}=240{ }^{\circ} \mathrm{C}$ )

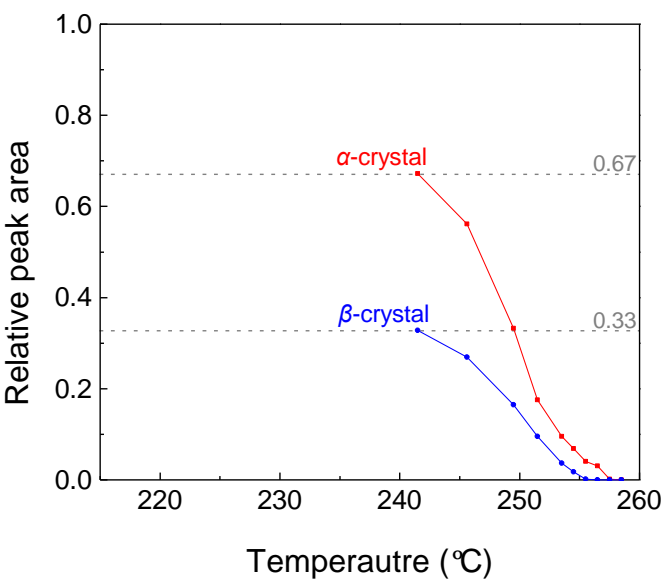

Figure S11. Schematic peak deconvolution WAXS profiles for sPS-21\%3MS cold-crystallization at (a) 220 , (b) 230 , and (c) $240{ }^{\circ} \mathrm{C}$. Black dots are the experimental profiles, and red lines are the fitting profiles composed of two amorphous halos and crystalline peaks (black solid line). From (d) to (f) show the evolution of relative crystallinity between $\alpha$ - and $\beta$-crystals upon heating for $T_{c}=(\mathrm{d})$ 220 , (e) 230 , and (f) $240{ }^{\circ} \mathrm{C}$, and the results for each specimen are normalized with the initial value respectively. The relative crystallinity indicates that the $\alpha$-crystals always have higher amount than $\beta$-crystals upon heating. 
(a)

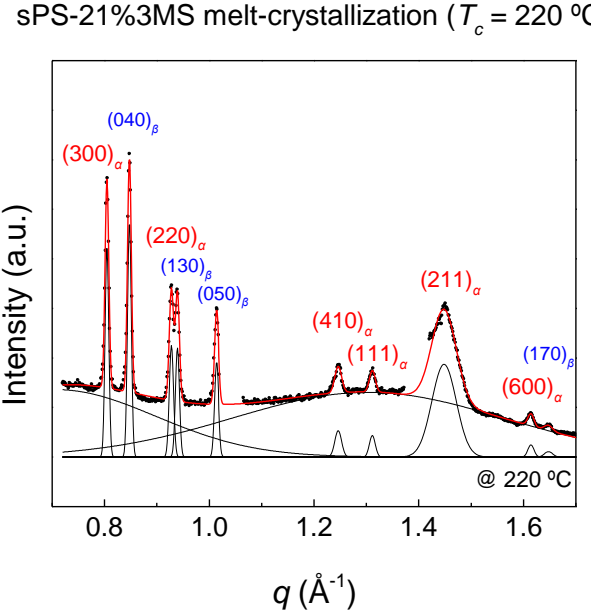

(b)

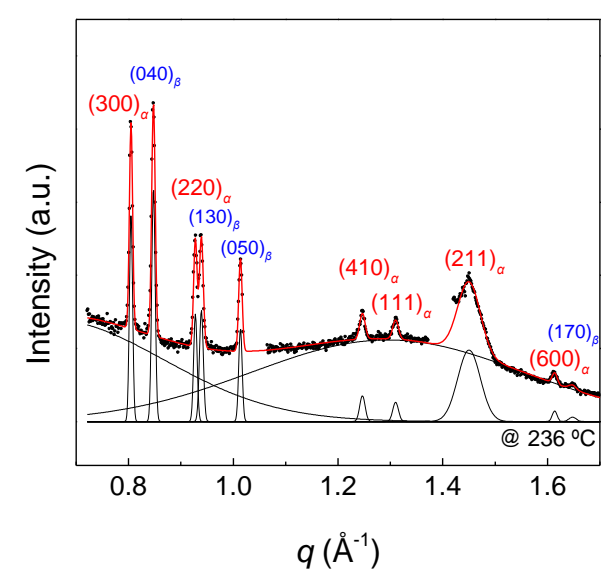

(c) sPS-21\%3MS melt-crystallization $\left(T_{c}=220^{\circ} \mathrm{C}\right)$

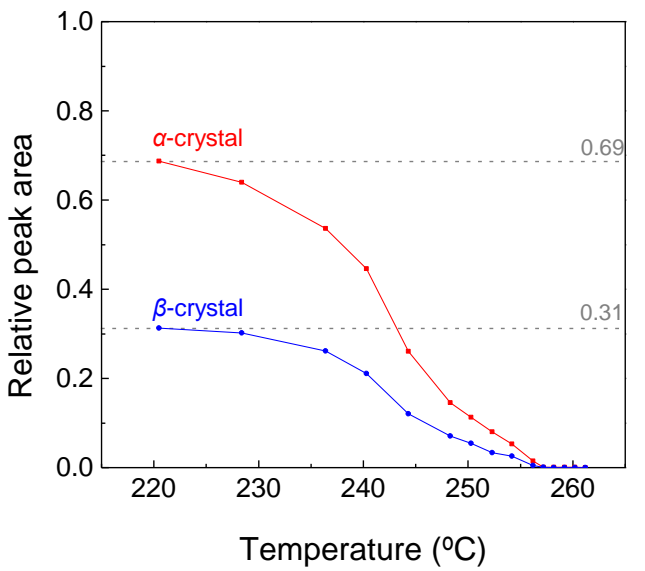

(d) sPS-21\%3MS melt-crystallization $\left(T_{c}=235^{\circ} \mathrm{C}\right)$

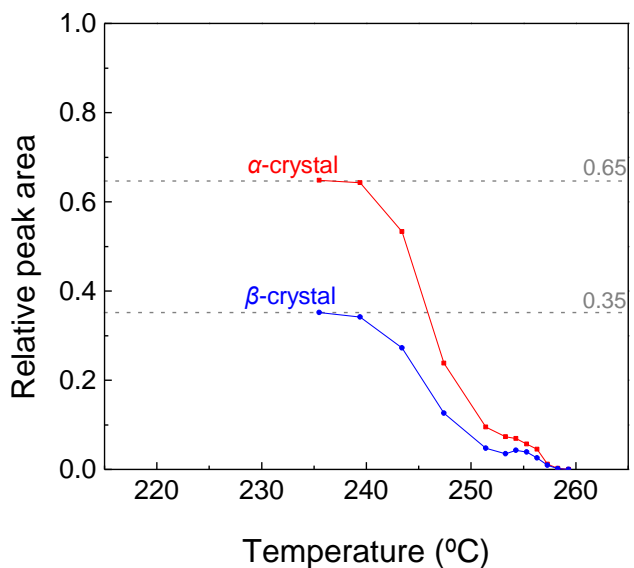

Figure S12. Schematic peak deconvolution WAXS profiles for sPS-21\%3MS melt-crystallization at (a) 220 and (b) $235^{\circ} \mathrm{C}$. Black dots are the experimental profiles, and red lines are the fitting profiles composed of two amorphous halos and crystalline peaks (black solid line). (c) and (d) are the evolution of relative peak area between $\alpha$ - and $\beta$-crystals upon heating, and the results are normalized with the initial value. The relative crystallinity indicates that the $\alpha$-crystals always have higher amount than $\beta$-crystals upon heating.

After subtracting the molten state scattering profiles, the reduced sPS-21\%3MS cold-and melt-crystallized SAXS heating profiles that approached melting are shown in Figure S13-14. Open symbols are the experimental data, and the solid lines are the fitting result by the use of the cylinder-shaped form factor with disperse radius. The corresponding fitting parameters of the radius and thickness are presented in Figure S15, and the relation between reciprocal crystal thickness $\left(l_{c}^{-1}\right)$ and the experimental temperature is illustrated in Figure S16. The melting line can be constructed by extrapolating the data points (solid symbol) which is just several degrees below melting, and for the case of $l_{c}{ }^{-1} \sim 0$ gives the equilibrium melting temperature $\left(T_{m}{ }^{0}\right)$ of sPS$21 \% 3 \mathrm{MS} \alpha$-crystal as $260.9 \pm 0.7^{\circ} \mathrm{C}$. Based on the depression level of $T_{m}{ }^{0}$ and the slope of the melting line, the penalty energy $(\varepsilon)$ and the fold surface energy $\left(\sigma_{e}\right)$ can be derived via SanchezEby and Gibbs-Thomson theory, which is $\varepsilon=26.4 \pm 2.4 \mathrm{MJ} \mathrm{m}^{-3}$ and $\sigma_{e}=4.5 \pm 0.8 \mathrm{~mJ} \mathrm{~m}^{-2}$. 
(a) SPS-21\%3MS (cold-crystallized, $T_{c}=220 \stackrel{\circ}{\circ}$ )

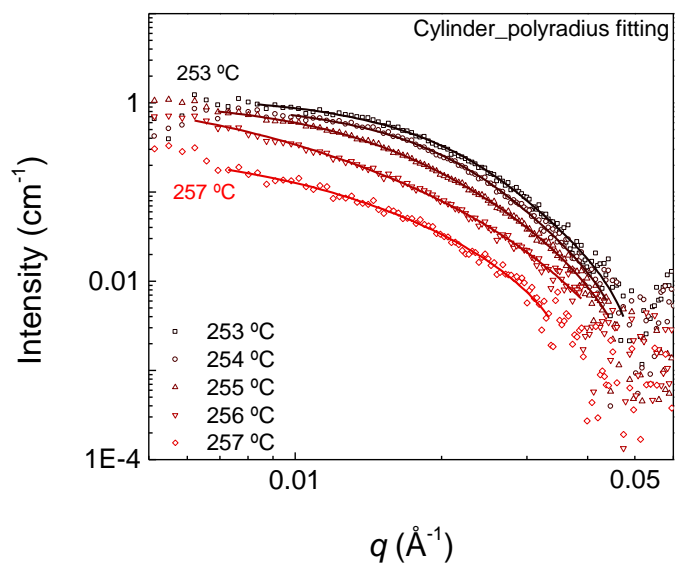

(c) sPS-21\%3MS (cold-crystallized, $T_{c}=240 \stackrel{\circ}{\circ}$ )

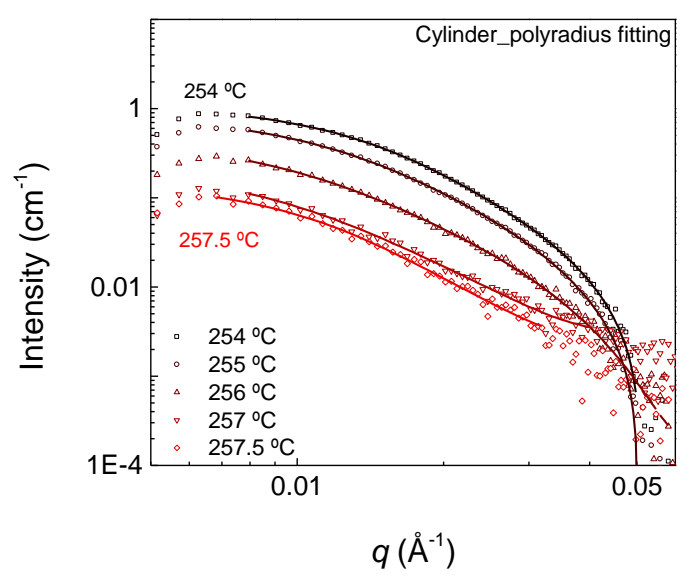

(b) $\quad$ SPS-21\%3MS (cold-crystallized, $T_{c}=230^{\circ} \mathrm{C}$ )

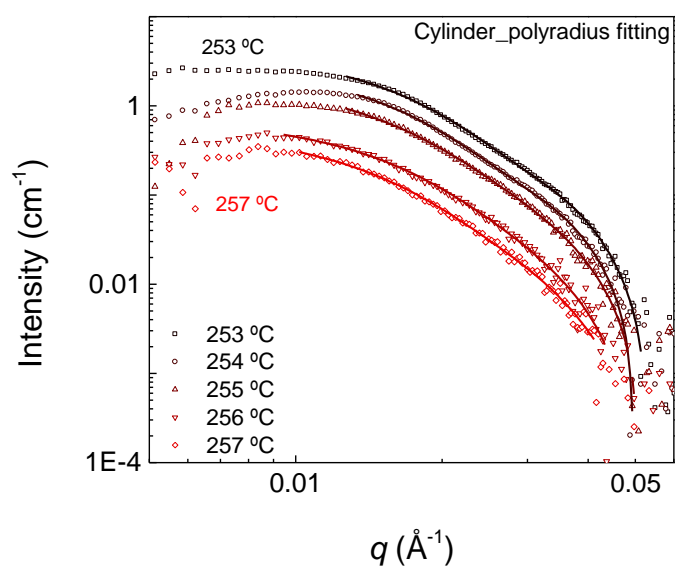

Figure S13. Reduced SAXS heating profiles of sPS-21\%3MS cold-crystallized at $T_{c}=$ (a) 220 , (b) 230 , and (c) $240{ }^{\circ} \mathrm{C}$ before melting (ca. 253 to $258^{\circ} \mathrm{C}$ ), which generally gave only the form factor scattering. The solid lines represent the fitting profiles by using the cylinder-shaped form factor with polydisperse radius.
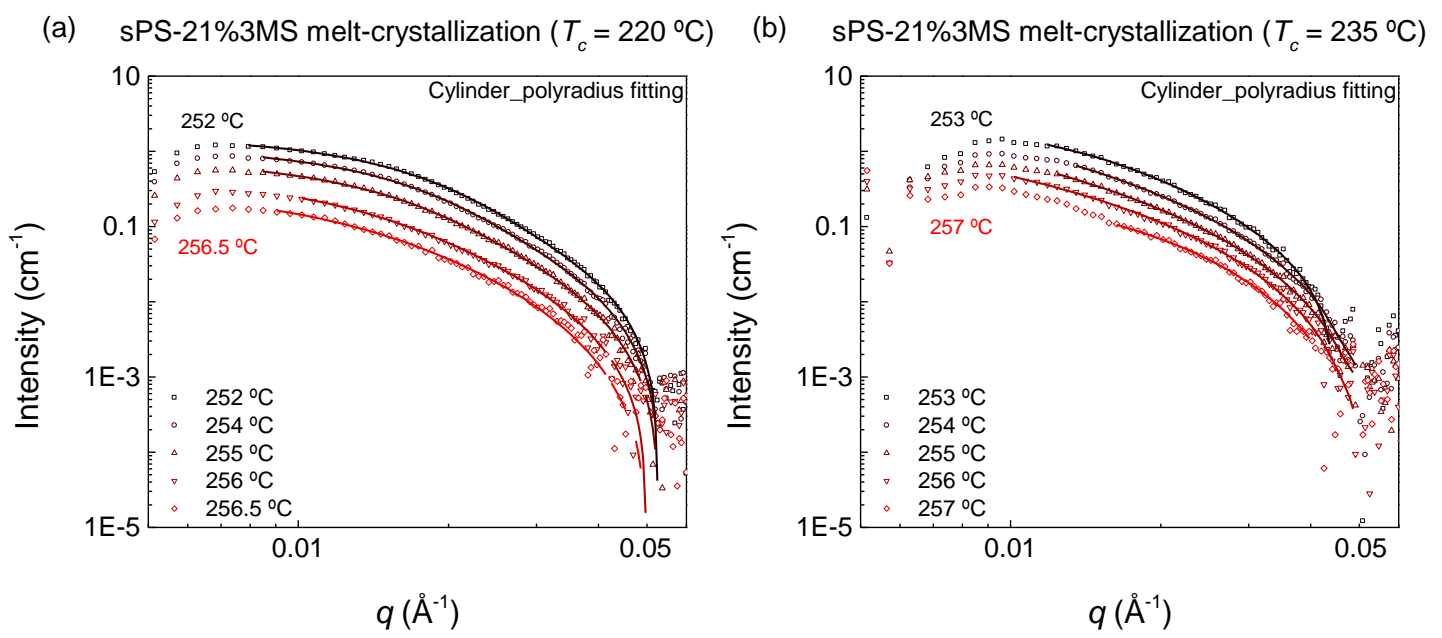

Figure S14. Reduced SAXS heating profiles of sPS-21\%3MS melt-crystallized at $T_{c}=$ (a) 220 and (b) $235^{\circ} \mathrm{C}$ before melting (in ca. $252-257^{\circ} \mathrm{C}$ ), which generally showed form factor scattering. Solid lines are the fitting profiles by using cylinder shape with polydisperse radius form factor model. 
(a) sPS-21\%3MS fitting result (radius)

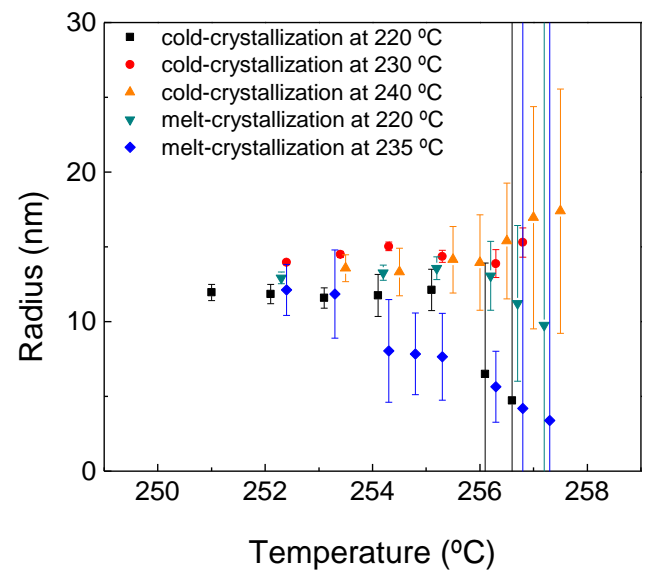

(b) sPS-21\%3MS fitting result (thickness)

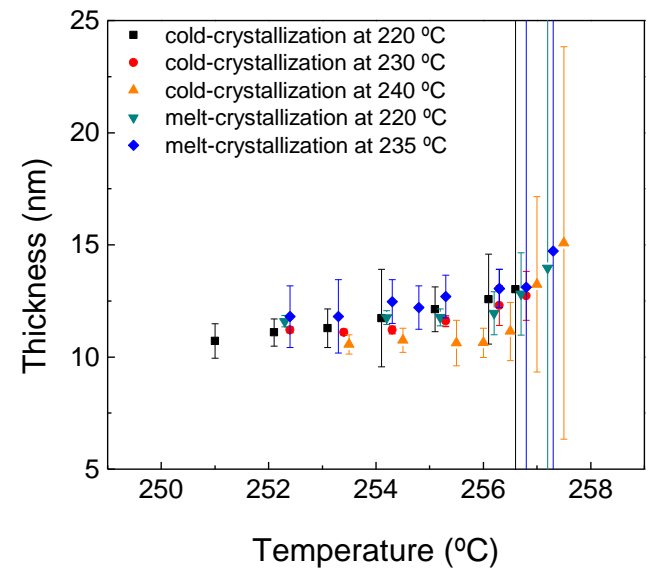

Figure S15. Evolution of (a) radius and (b) thickness of the cylinder-shaped nanograins upon heating, which were obtained from the reduced SAXS profiles in Figure S13-14. Depending on the size of radius and thickness, the nanograins are close to the disk-like structures.

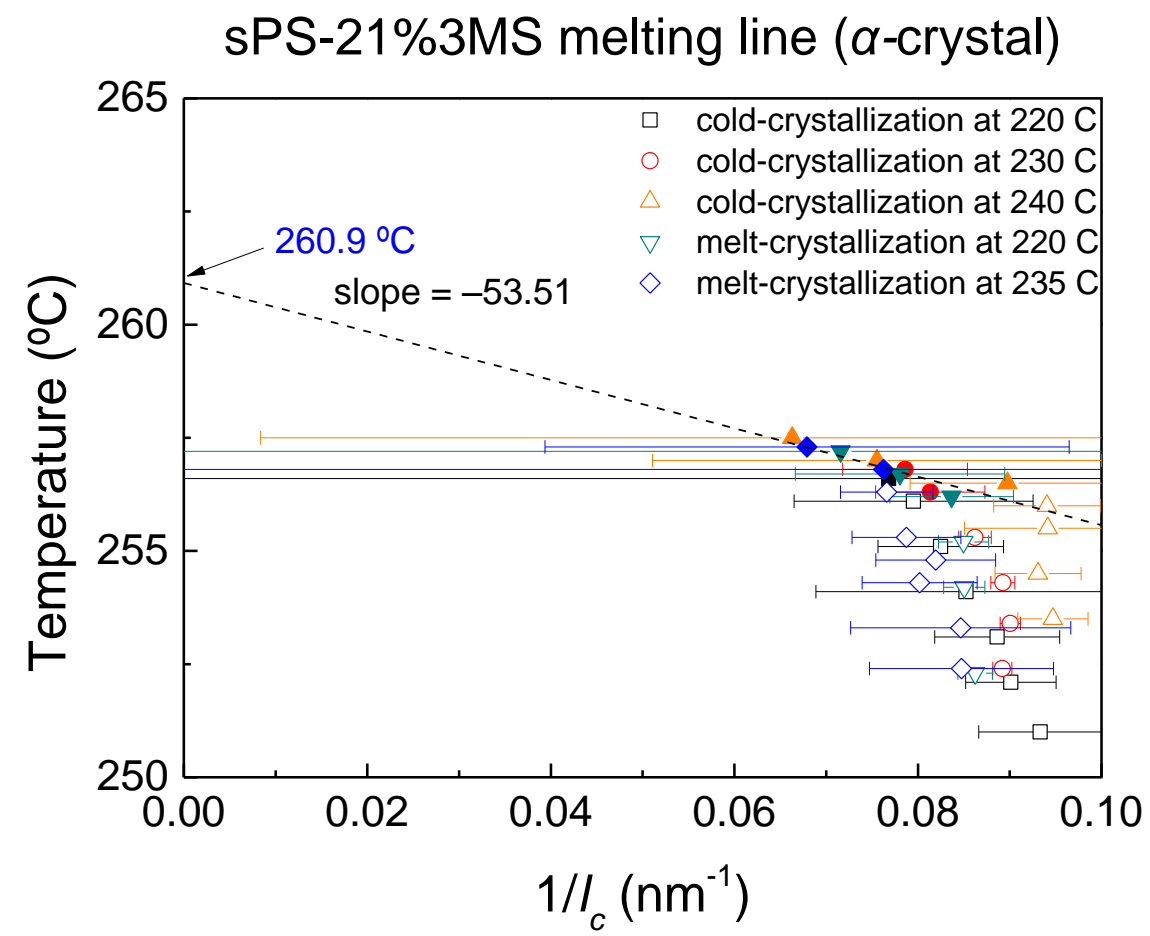

Figure S16. Evolution of the reciprocal crystal thickness $\left(1 / l_{c}\right)$ upon heating for sPS-21\%3MS coldand melt-crystallized specimens. Dash line represents the melting line that was established by extrapolating the data points with solid symbols (higher than ca. $256.2^{\circ} \mathrm{C}$ ), giving the equilibrium melting temperature $\left(T_{m}{ }^{0}\right)$ for sPS-21\%3MS cold-crystallized $\alpha$-crystals as $260.9 \pm 0.7^{\circ} \mathrm{C}$. 
The simultaneous SAXS/WAXS heating profiles of sPS-5\%3MS melt-crystallized specimens are illustrated in Figure S17. Corresponding WAXS profiles in Figure S17d-f show that specimens were all formed $\beta$-dominated crystal. Upon heating, the lamellar peak in SAXS profiles progressively become broader, and peak position $\left(q_{c}\right)$ shifts to lower $q$-position, which represents the increase of long-period and weakening of the spatial correlation between nanograins; in WAXS profiles, the reflection peaks gradually decrease without appearing any new reflection peak. Thus, the SAXS/WAXS profiles correspond to the melting behaviors of melt-crystallized sPS-5\%3MS specimens. Besides, after completely melt at ca. $270{ }^{\circ} \mathrm{C}$, the SAXS profiles (red sloid lines in Figure $\mathrm{S} 17 \mathrm{a}-\mathrm{c}$ ) give the power-law decrease scattering with exponent ca. -3 and only show amorphous profiles in the WAXS results. 
(a) $\quad$ PPS-5\%3MS melt-crystallization $\left(T_{c}=235 \stackrel{\circ}{\circ}\right)$

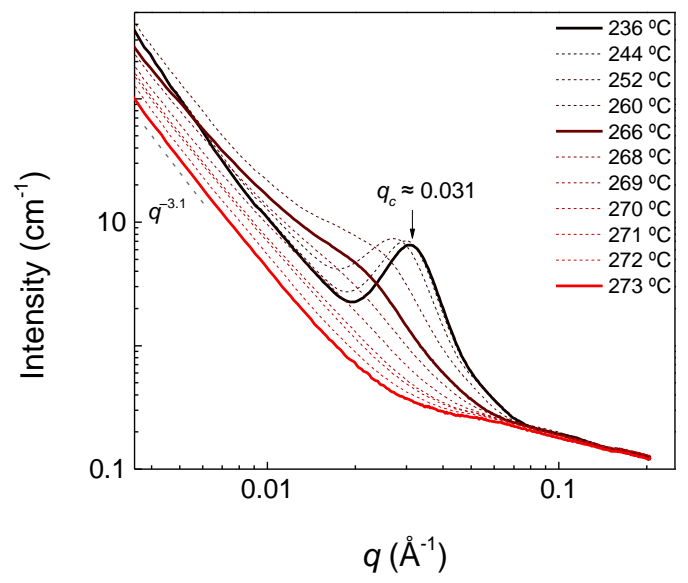

(b) SPS-5\%3MS melt-crystallization $\left(T_{c}=240^{\circ} \mathrm{C}\right)$

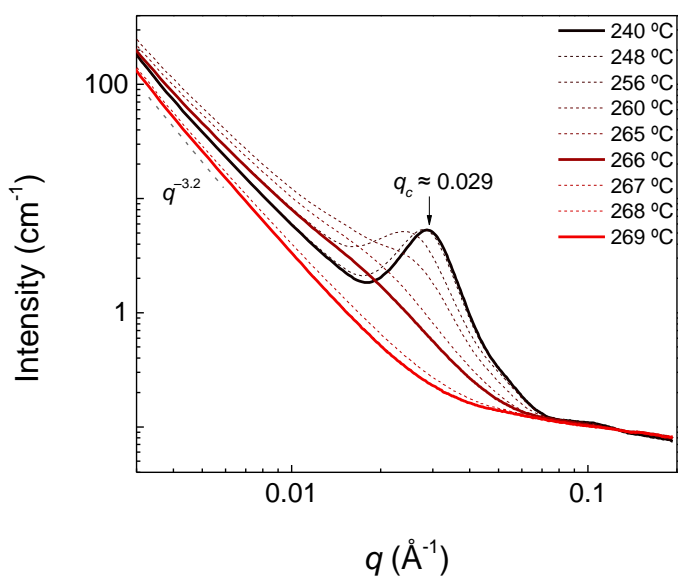

(c) $\quad$ SPS-5\%3MS melt-crystallization $\left(T_{c}=245{ }^{\circ} \mathrm{C}\right)$

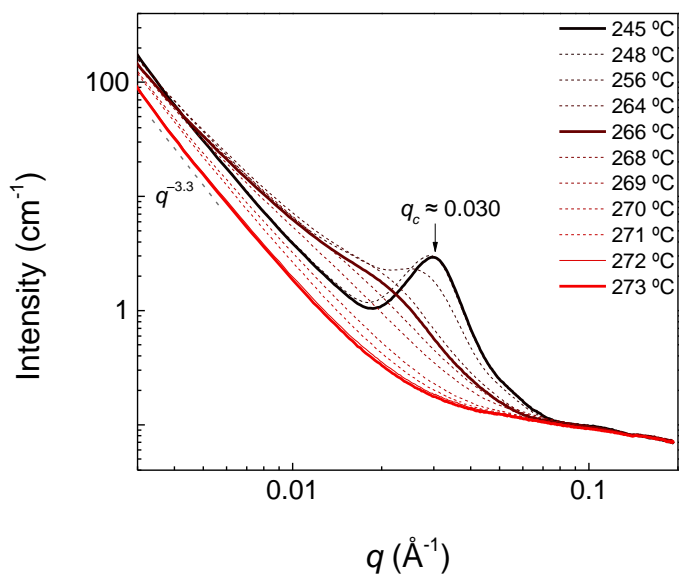

(d) $\quad$ sPS-5\%3MS melt-crystallization $\left(T_{c}=235 \stackrel{\circ}{ } \mathrm{C}\right)$

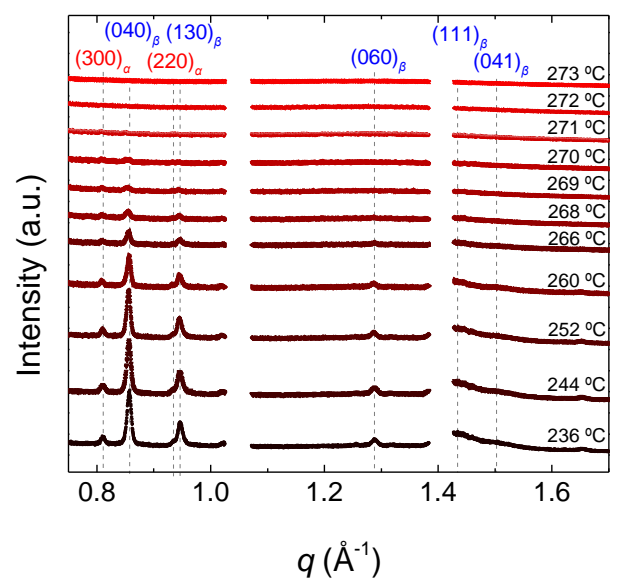

(e) sPS-5\%3MS melt-crystallization $\left(T_{c}=240^{\circ} \mathrm{C}\right)$

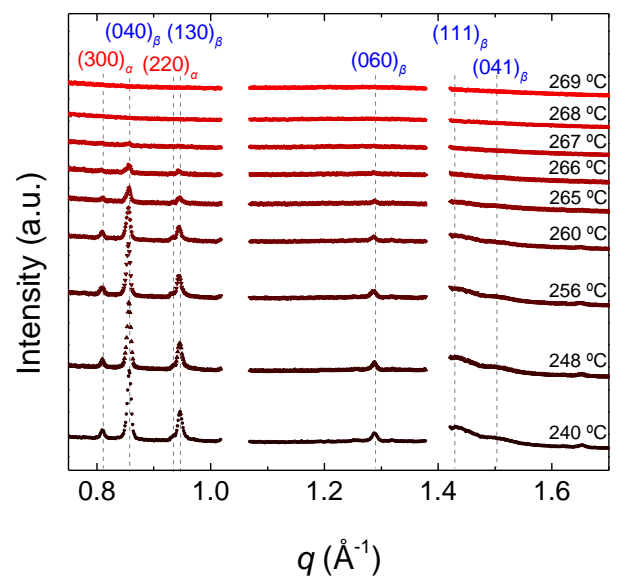

(f) $\quad$ SPS-5\%3MS melt-crystallization $\left(T_{c}=245^{\circ} \mathrm{C}\right)$

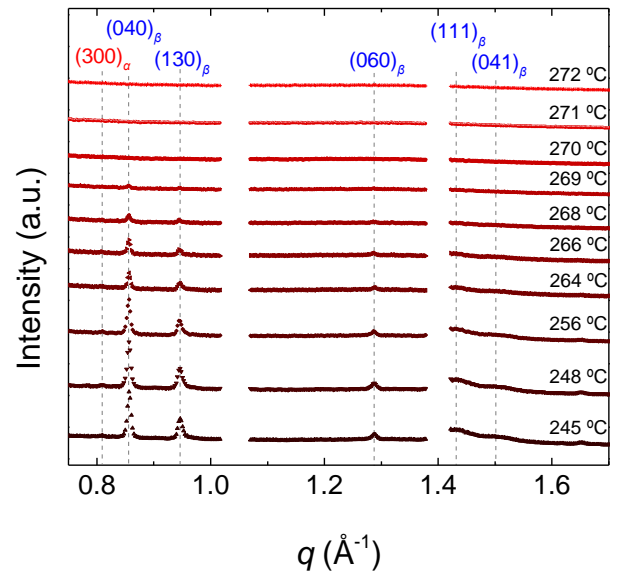

Figure S17. Simultaneous (a)-(c) SAXS and (d)-(f) WAXS heating profiles of sPS-5\%3MS meltcrystallized specimens ( $\beta$-dominated crystals) from each $T_{c}$ to molten state. Black solid lines in the SAXS profiles represent the starting profiles (at $T_{c}$ ) for each specimen, and the red solid lines stand for the scattering profile of molten state. The WAXS profiles show that the characteristic reflections gradually decrease and completely melt after reaching ca. $270{ }^{\circ} \mathrm{C}$, which gave only amorphous halo.

Illustrated in Figure S18a-c are the schematic peak deconvoluted WAXS profiles, and the relative peak area presented in Figure S18d-f were calculated based on the deconvoluted results with normalizing to initial value respectively. Depending on the evolution of relative peak area, the 
$\beta$-crystals always have higher population than $\alpha$-crystals and populations of both $\alpha$-and $\beta$-crystals decrease progressively upon heating. Thus, the melting process dominates the heating process.

(a) sPS-5\%3MS (melt-crystallized, $T_{c}=235^{\circ} \mathrm{C}$ )

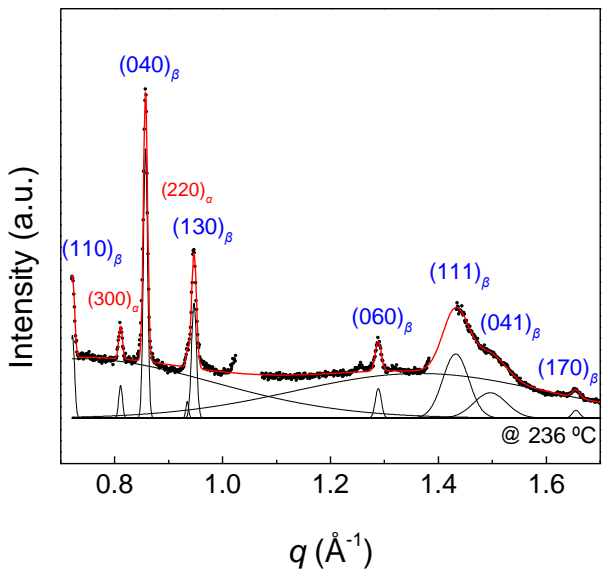

(b) sPS-5\%3MS (melt-crystallized, $T_{c}=240^{\circ} \mathrm{C}$ )

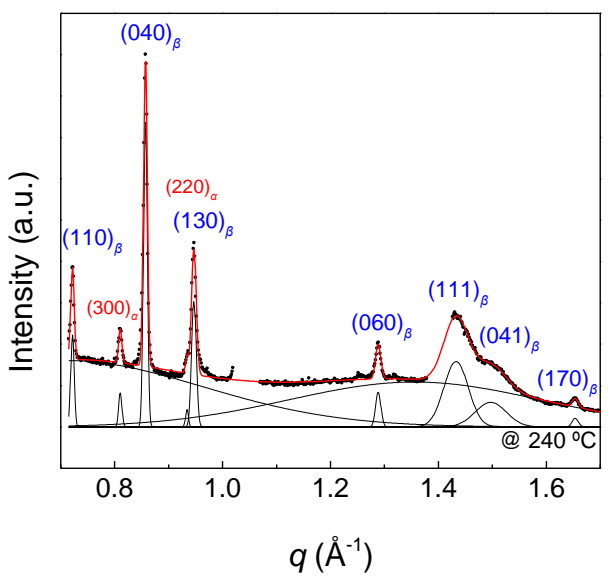

(c) $\quad$ SPS-5\%3MS (melt-crystallized, $T_{c}=245^{\circ} \mathrm{C}$ )

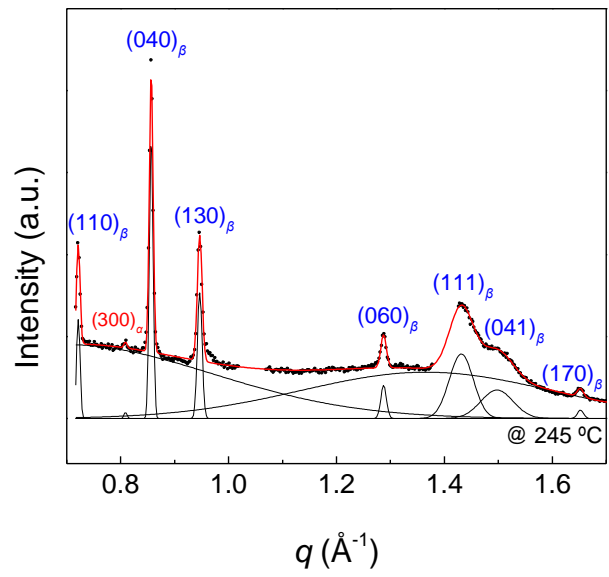

(d) $\quad$ SPS-5\%3MS (melt-crystallized, $T_{c}=235^{\circ} \mathrm{C}$ )

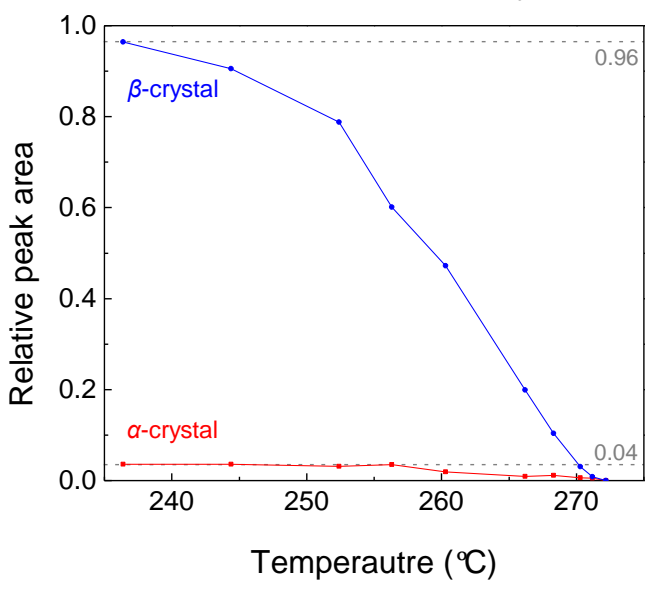

(e) $\quad$ SPS-5\%3MS (melt-crystallized, $T_{c}=240{ }^{\circ} \mathrm{C}$ )

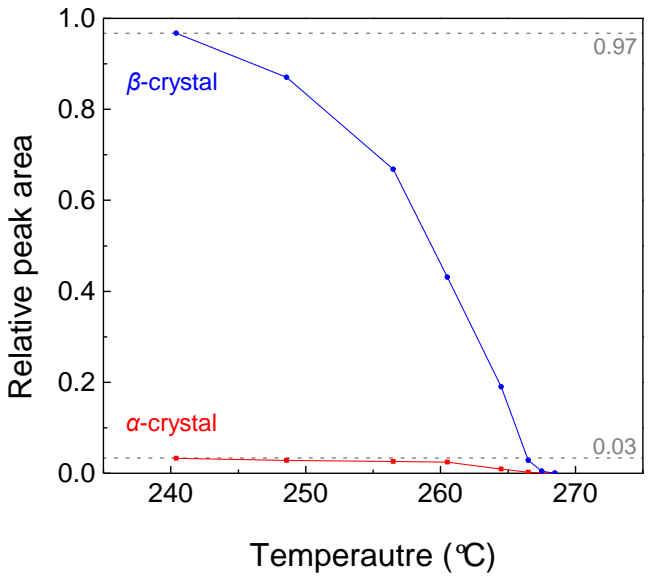

(f) $\quad$ sPS-5\%3MS (melt-crystallized, $T_{c}=245^{\circ} \mathrm{C}$ )

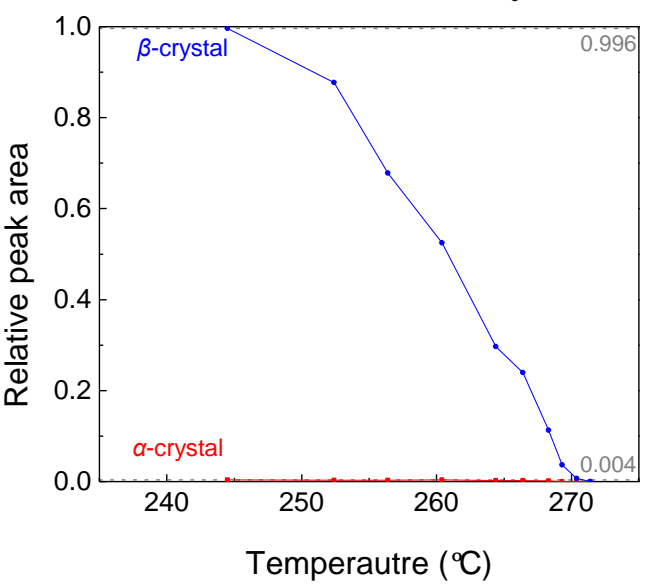

Figure S18. The schematic peak deconvolution WAXS profiles of sPS-5\%3MS melt-crystallized specimen at $T_{c}=$ (a) 235 , (b) 240 , and (c) $245^{\circ} \mathrm{C}$. Black dots are the experimental data, and the red lines are the fitting profiles, which reveals that the profiles consist of two amorphous halos and crystalline diffraction peaks of $\alpha$ - and $\beta$-crystals. (d)-(f) are the evolutions of relatively peak area between $\alpha$ - and $\beta$-crystals upon heating, and the results were normalized with the initial values for each specimen respectively, which denotes that the $\beta$-crystals always dominate the crystalline population until melting. 
After subtracting the molten state scattering profiles, the reduced sPS-5\%3MS meltcrystallized SAXS heating profiles that approached melting are shown in Figure S19. Open symbols are the experimental data, and the solid lines are the fitting result by the use of the cylinder-shaped form factor with disperse radius. The corresponding fitting parameters of the radius and thickness are presented in Figure S20, and the relation between reciprocal crystal thickness $\left(l_{c}{ }^{-1}\right)$ and the experimental temperature is illustrated in Figure S21. The melting line can be constructed by extrapolating the data points (solid symbol), which is just several degrees below melting, and for the case of $l_{c}{ }^{-1} \sim 0$ gives the equilibrium melting temperature $\left(T_{m}{ }^{0}\right)$ of sPS-5\%3MS $\beta$-crystal as $287.5 \pm 2.7^{\circ} \mathrm{C}$. Based on the depression level of $T_{m}{ }^{0}$ and the slope of the melting line, the penalty energy $(\varepsilon)$ and the fold surface energy $\left(\sigma_{e}\right)$ can be derived via Sanchez-Eby and Gibbs-Thomson theory, which is $\varepsilon=77.5 \pm 11.1 \mathrm{MJ} \mathrm{m}^{-3}$ and $\sigma_{e}=22.5 \pm 7.0 \mathrm{~mJ} \mathrm{~m}^{-2}$.

(a) $\quad$ SPS-5\%3MS (melt-crystallized, $T_{c}=235^{\circ} \mathrm{C}$ )

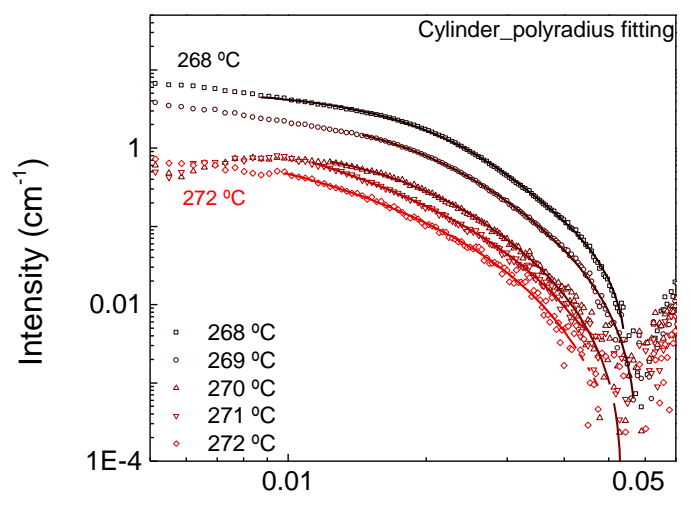

$q\left(\AA^{-1}\right)$

(c) $\quad$ SPS-5\%3MS (melt-crystallized, $T_{c}=245^{\circ} \mathrm{C}$ )

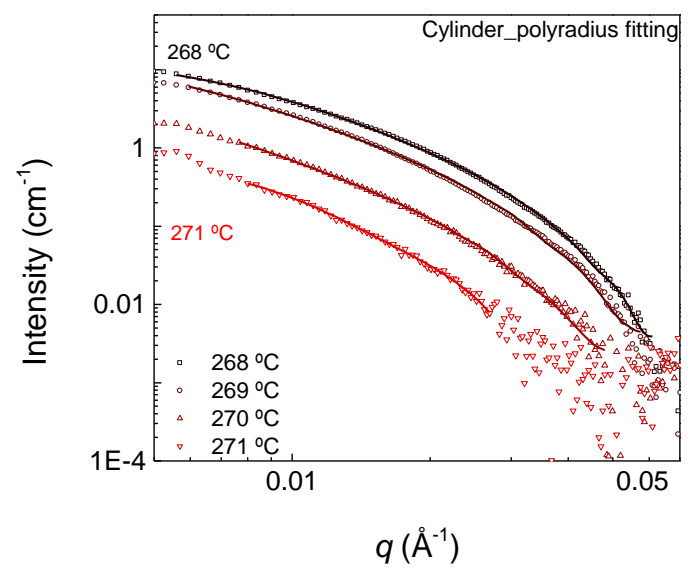

(b) $\quad$ SPS-5\%3MS (melt-crystallized, $T_{c}=240 \stackrel{\circ}{\circ}$ )

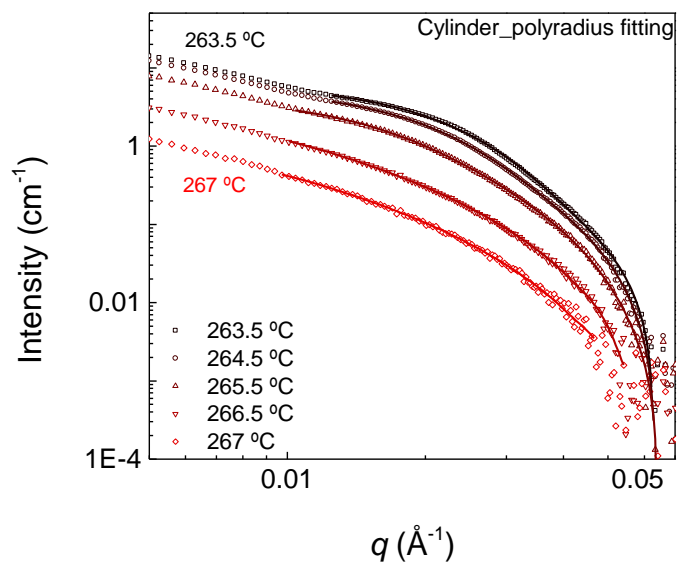

Figure S19. The reduced SAXS heating profiles of sPS-5\%3MS melt-crystallized specimens at $T_{c}=$ (a) 235 , (b) 240 , and (c) $245^{\circ} \mathrm{C}$ before melting (in ca. 264 to $272{ }^{\circ} \mathrm{C}$ temperature range), which generally gave only form factor scattering. The solid lines are the fitting profiles by using the cylinder-shaped form factor with polydisperse radius. 

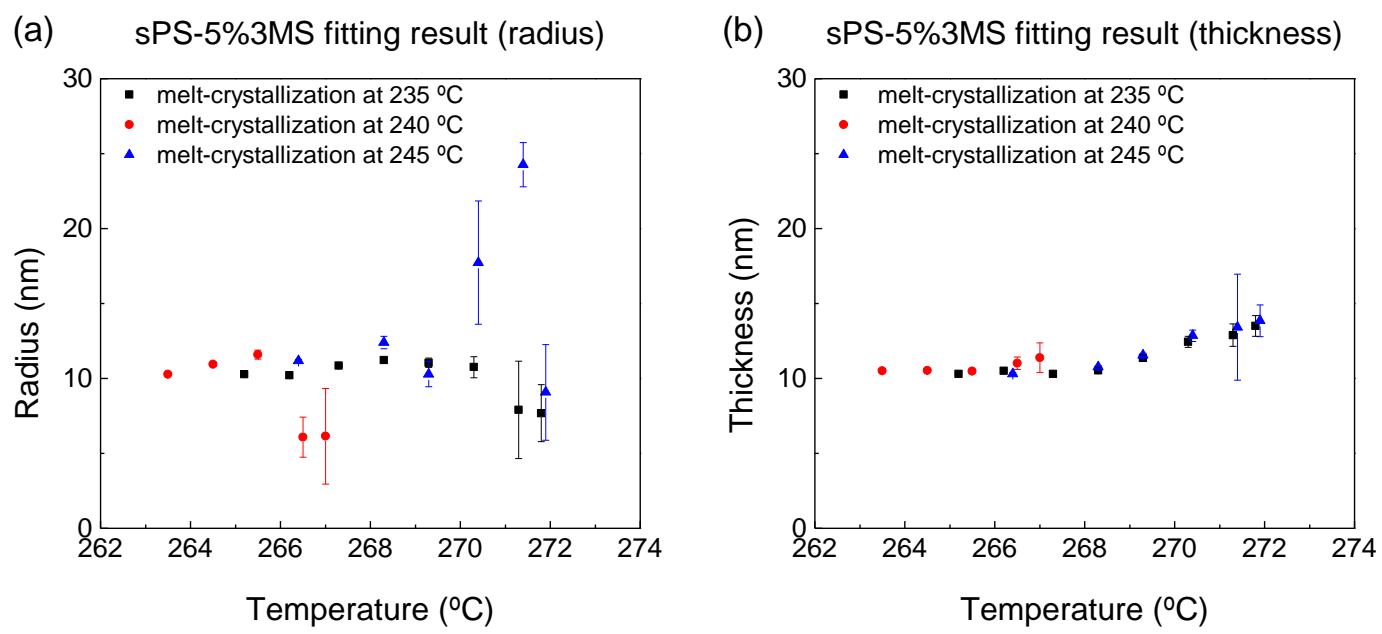

Figure S20. The evolution of (a) radius and (b) thickness of the nanograins during the heating scan which obtained from the reduced SAXS fitting profiles in Figure S19. Based on the fitting parameters, the nanograins are close to disk-like structures.

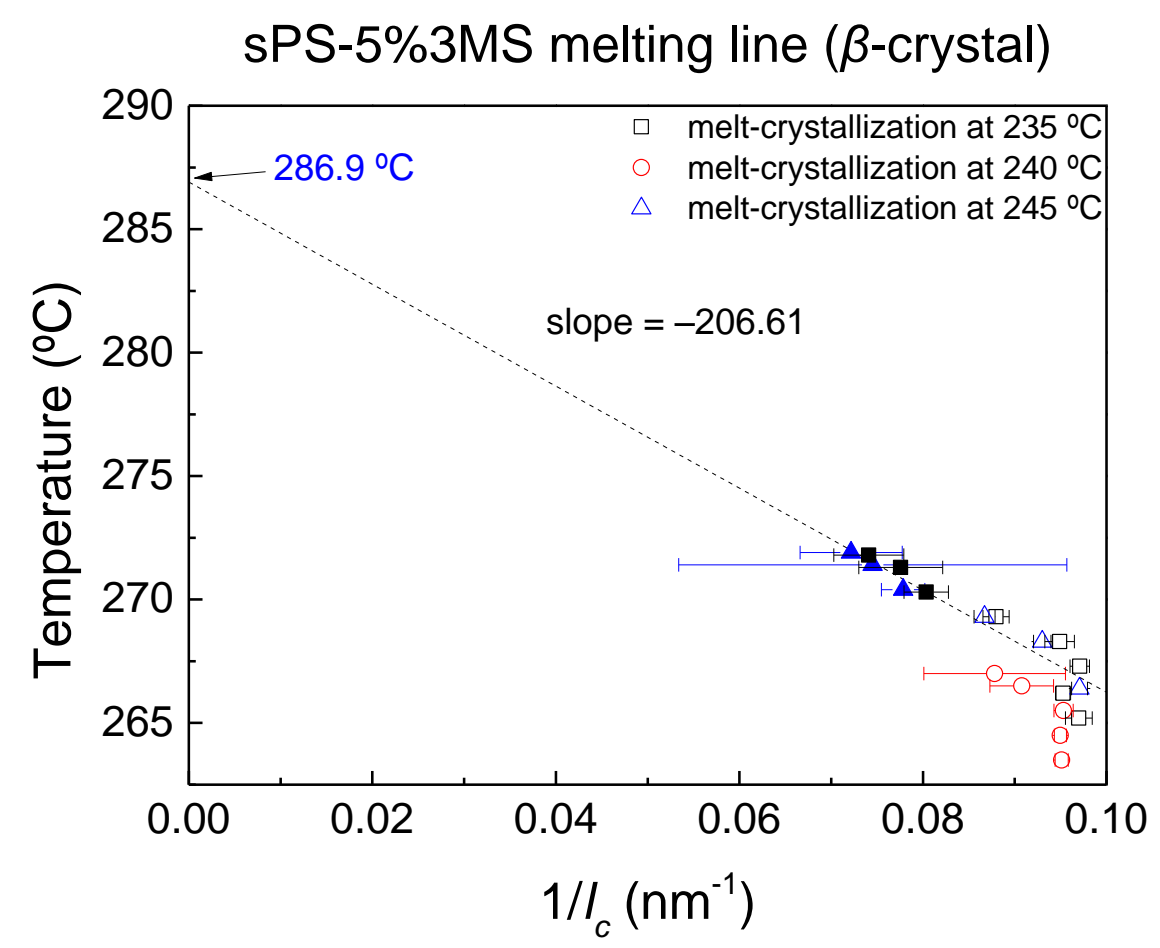

Figure S21. The evolution of the reciprocal crystal thickness $\left(1 / l_{c}\right)$ upon heating for sPS-5\%3MS melt-crystallized specimens. Dash line represents the melting line that established by extrapolating the data points, which were higher than $270{ }^{\circ} \mathrm{C}$ (solid symbols), giving the equilibrium melting temperature $\left(T_{m}{ }^{0}\right)$ for sPS-5\%3MS $\beta$-crystals as $286.9 \pm 3.7^{\circ} \mathrm{C}$. 
The simultaneous SAXS/WAXS heating profiles of sPS-11\%3MS melt-crystallized specimens are illustrated in Figure S22. Compared among each initial scattering profile (black solid lines), the lamellar peak position $q_{c}$ shifts to lower- $q$ value with increasing $T_{c}$, implying that higher isothermal temperature gives larger long period (inter-particle distance). Corresponding WAXS profiles in Figure $\mathrm{S} 22 \mathrm{~d}-\mathrm{f}$ show that specimens were all formed $\beta$-dominated crystal. Upon heating, the lamellar peak in SAXS profiles progressively become broader and $q_{c}$ shifts to lower $q$-position, which represents the increase of long-period and weakening of the spatial correlation between nanograins; in WAXS profiles, the reflection peaks gradually decrease without appearing any new reflection peak. Thus, the SAXS/WAXS profiles correspond to the melting behaviors of meltcrystallized sPS-11\%3MS specimens. Besides, after completely melt at ca. $263^{\circ} \mathrm{C}$, the SAXS profiles (red sloid lines in Figure S22a-c) give the power-law decrease scattering with exponent ca. -3 and only show amorphous profiles in the WAXS results. 
(a) sPS-11\%3MS melt-crystallization $\left(T_{c}=220 \stackrel{\circ}{\circ}\right)$

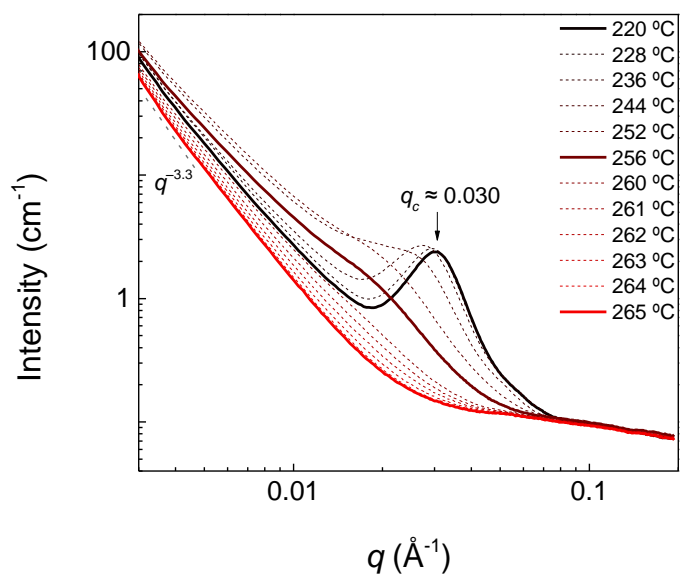

(b) sPS-11\%3MS melt-crystallization $\left(T_{c}=236 \stackrel{\circ}{\circ}\right)$

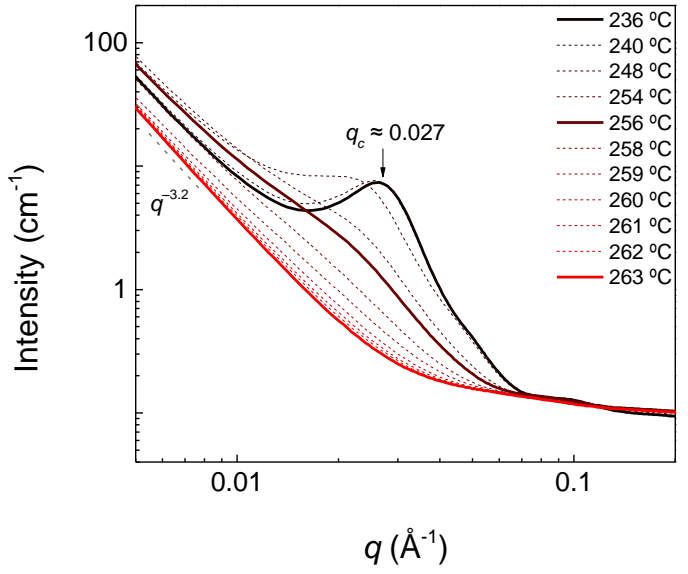

(c) sPS-11\%3MS melt-crystallization $\left(T_{c}=240 \stackrel{\circ}{\circ}\right)$

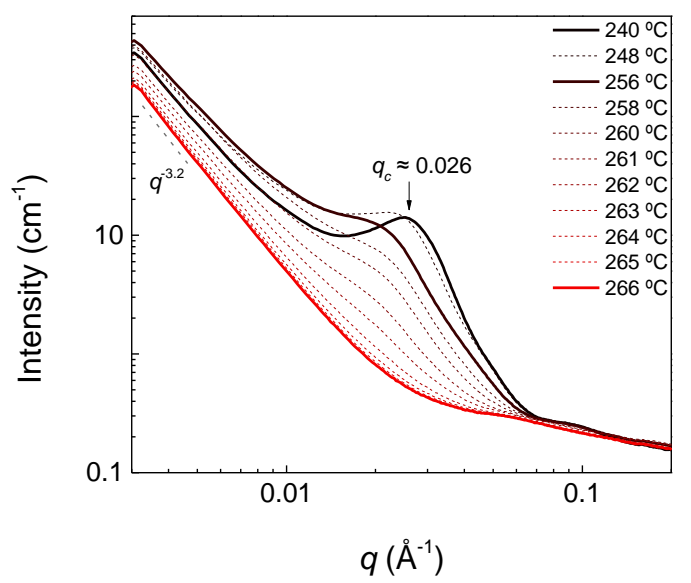

(d) sPS-11\%3MS melt-crystallization $\left(T_{c}=220 \stackrel{\circ}{C}\right)$

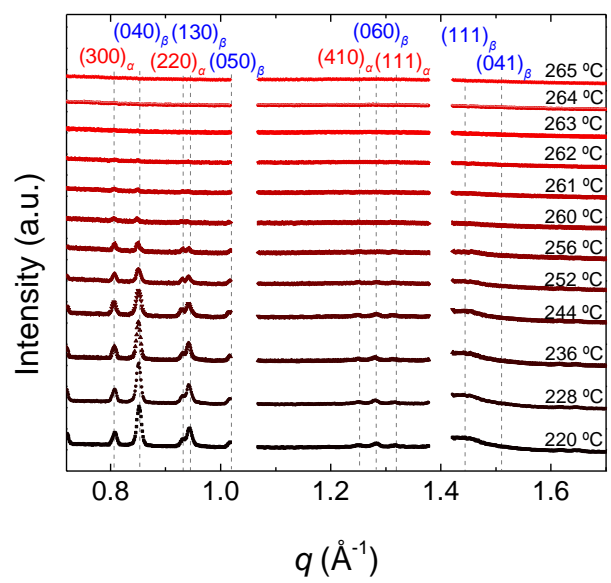

(e) $\mathrm{sPS}-11 \% 3 \mathrm{MS}$ melt-crystallization $\left(T_{c}=236^{\circ} \mathrm{C}\right)$

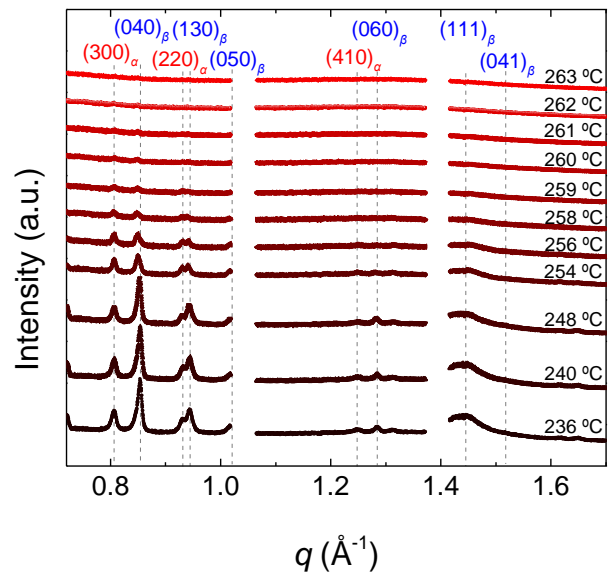

(f) sPS-11\%3MS melt-crystallization $\left(T_{c}=240^{\circ} \mathrm{C}\right)$

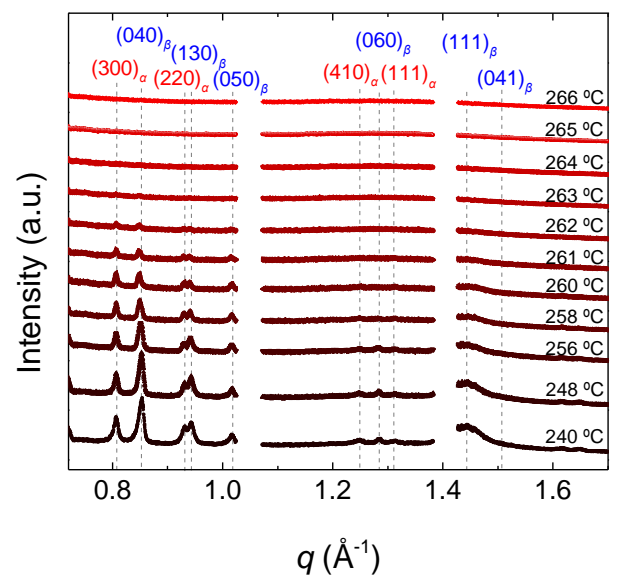

Figure S22. Simultaneous (a)-(c) SAXS and (d)-(f) WAXS heating profiles of sPS-11\%3MS meltcrystallized specimens ( $\beta$-dominated crystals) that were heating from each $T_{c}$ to the molten state. Black solid lines in the SAXS profiles represent the starting profiles (at $T_{c}$ ) for each specimen, and the red solid lines describe the scattering profile of molten state. The WAXS profiles show that the reflections of characteristic crystalline plane gradually decrease and completely melt after reaching ca. $263{ }^{\circ} \mathrm{C}$ which gives amorphous profiles.

The schematic peak deconvoluted WAXS profiles of sPS-11\%3MS melt-crystallized specimens are illustrated in Figure S23a-c, and the evolutions of relative peak area are presented Figure S23d-f, which were calculated based on the deconvoluted results with normalizing to initial 
value respectively. Among the heating process, the $\beta$-crystals always have higher population than $\alpha$ crystals and populations of both $\alpha$ - and $\beta$-crystals decrease progressively. Thus, the melting process dominates upon heating.

(a) sPS-11\%3MS (melt-crystallized, $T_{c}=220^{\circ} \mathrm{C}$ )

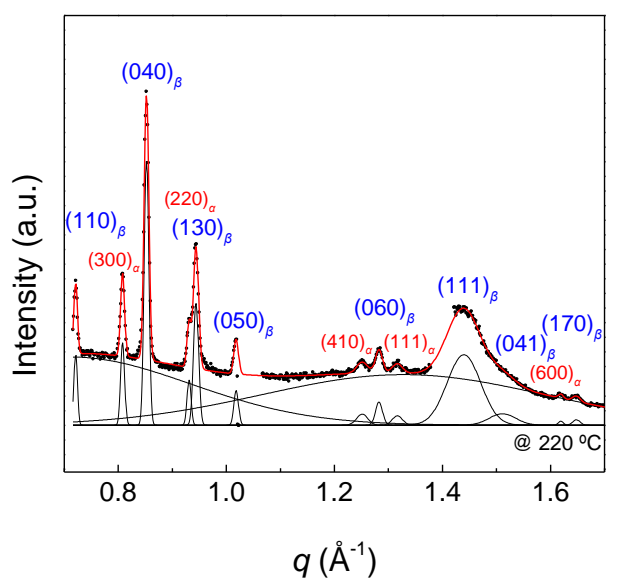

(b) sPS-11\%3MS (melt-crystallized, $T_{c}=236^{\circ} \mathrm{C}$ )

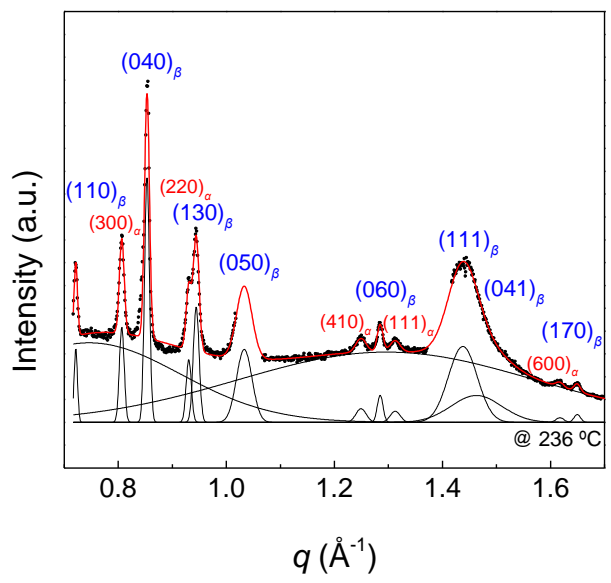

(c) sPS-11\%3MS (melt-crystallized, $T_{c}=240^{\circ} \mathrm{C}$ )

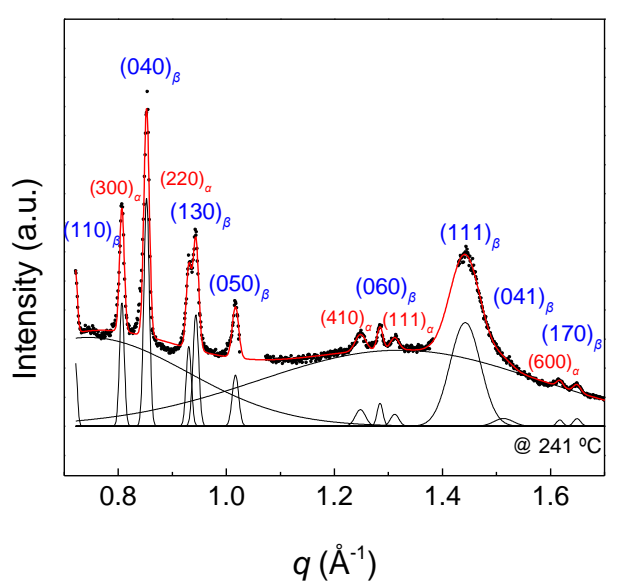

(d) sPS-11\%3MS (melt-crystallized, $T_{c}=220 \stackrel{\circ}{\circ}$ )

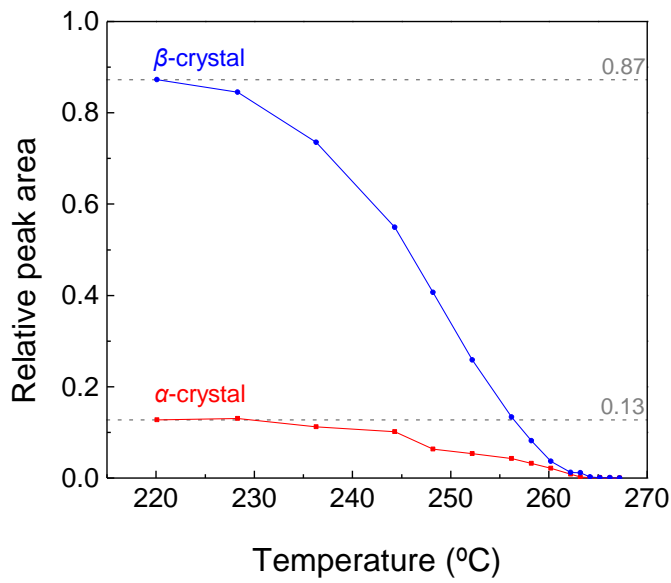

(e) sPS-11\%3MS (melt-crystallized, $T_{c}=236^{\circ} \mathrm{C}$ )

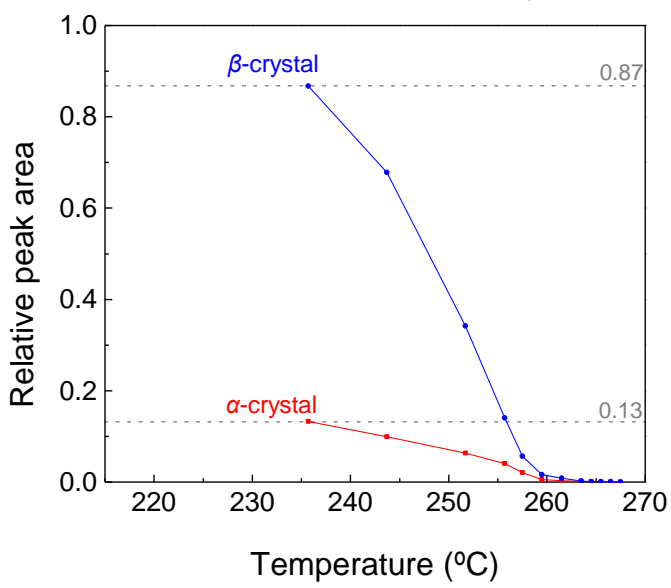

(f) $\mathrm{sPS}-11 \% 3 \mathrm{MS}$ (melt-crystallized, $T_{c}=240^{\circ} \mathrm{C}$ )

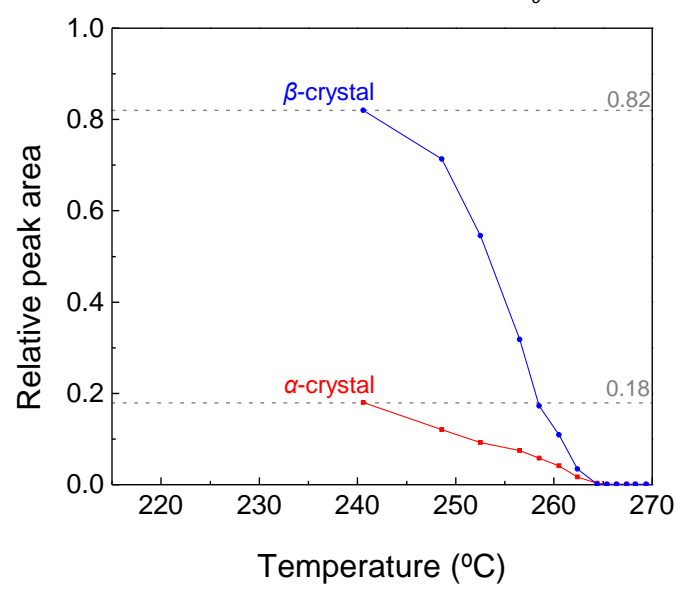

Figure S23. The schematic peak deconvolution WAXS profiles of sPS-11\%3MS melt-crystallized at $T_{c}=$ (a) 220 , (b) 236 , and (c) $240^{\circ} \mathrm{C}$. Black dots are the experimental data, and the red lines are the fitting profiles, which reveals that the profiles consist of two amorphous halos and crystalline diffraction peaks of $\alpha$ - and $\beta$-crystals. The evolutions of relatively crystalline area between $\alpha$ - and $\beta$ crystals upon heating are illustrated in (d)-(f), and the results were normalized with the initial 
values for each specimen respectively, which denotes that the $\beta$-crystals always dominate the crystalline population until melting.

After subtracting the molten state scattering profiles, the reduced sPS-11\%3MS meltcrystallized SAXS heating profiles that approached melting are shown in Figure S24. Open symbols are the experimental data, and the solid lines are the fitting result by the use of the cylinder-shaped form factor with disperse radius. The corresponding fitting parameters of the radius and thickness are presented in Figure S25, and the relation between reciprocal crystal thickness $\left(l_{c}{ }^{-1}\right)$ and the experimental temperature is illustrated in Figure S26. The melting line can be constructed by extrapolating the data points (solid symbol), which is just several degrees below melting, and for the case of $l_{c}{ }^{-1} \sim 0$ gives the equilibrium melting temperature $\left(T_{m}{ }^{0}\right)$ of sPS-11\%3MS $\beta$-crystal as $273.2 \pm 1.3{ }^{\circ} \mathrm{C}$. Based on the depression level of $T_{m}{ }^{0}$ and the slope of the melting line, the penalty energy $(\varepsilon)$ and the fold surface energy $\left(\sigma_{e}\right)$ can be derived via Sanchez-Eby and Gibbs-Thomson theory, which is $\varepsilon=62.5 \pm 5.3 \mathrm{MJ} \mathrm{m}^{-3}$ and $\sigma_{e}=13.4 \pm 3.5 \mathrm{~mJ} \mathrm{~m}^{-2}$.
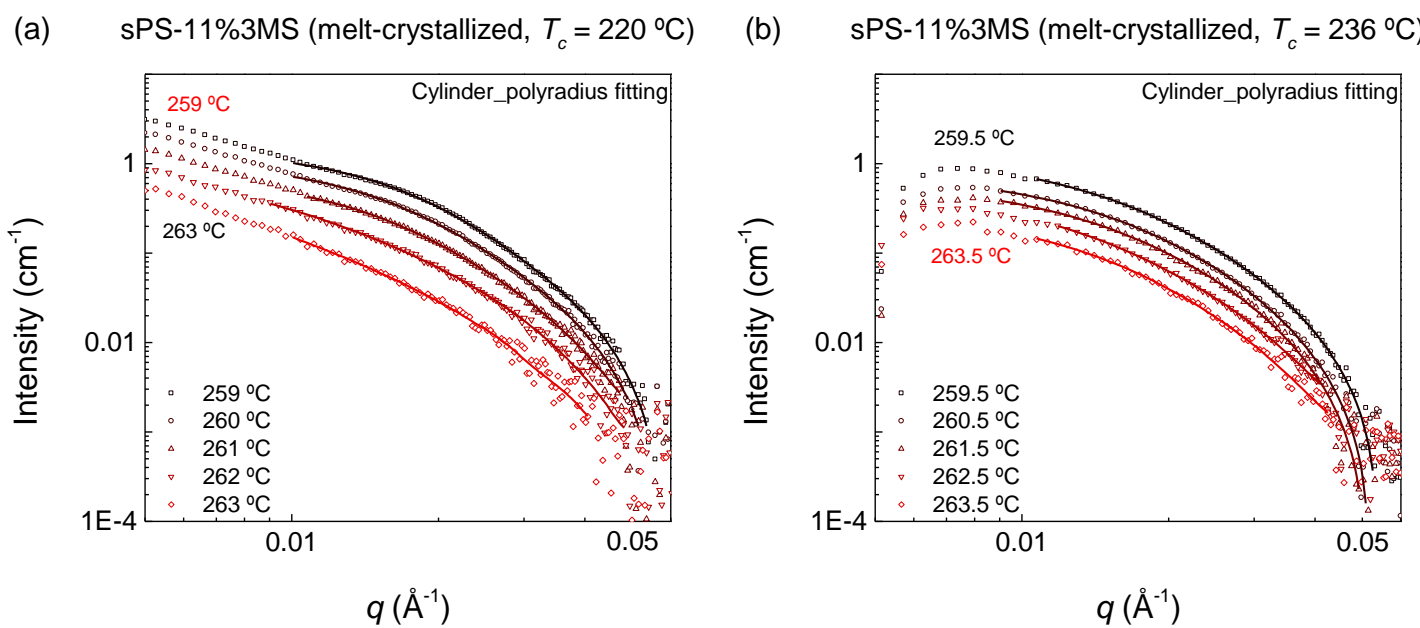

(c) SPS-11\%3MS (melt-crystallized, $T_{c}=240^{\circ} \mathrm{C}$ )

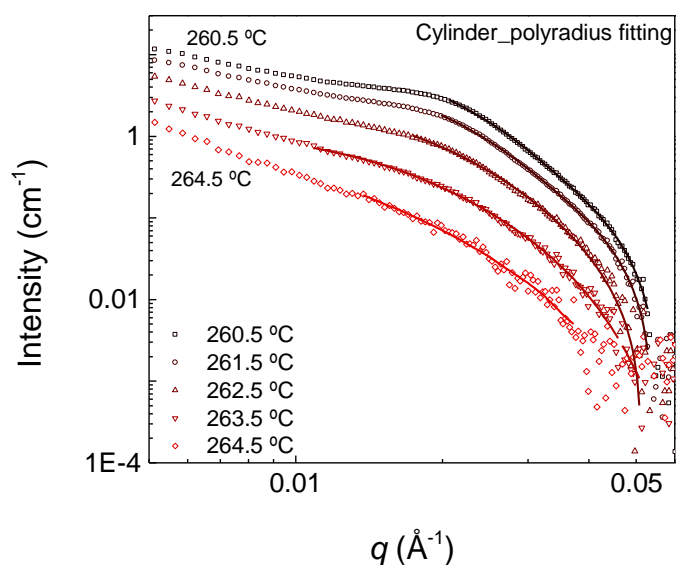

Figure S24. Reduced SAXS heating profiles of sPS-11\%3MS melt-crystallization at $T_{c}=$ (a) 220 , (b) 236 , and (c) $240{ }^{\circ} \mathrm{C}$ before melting (in ca. $259-265^{\circ} \mathrm{C}$ temperature range), which gives basically form factor scattering profiles. Solid lines are the fitting profiles by using cylinder with disperse radius form factor model. 
(a) SPS-11\%3MS fitting result (radius)

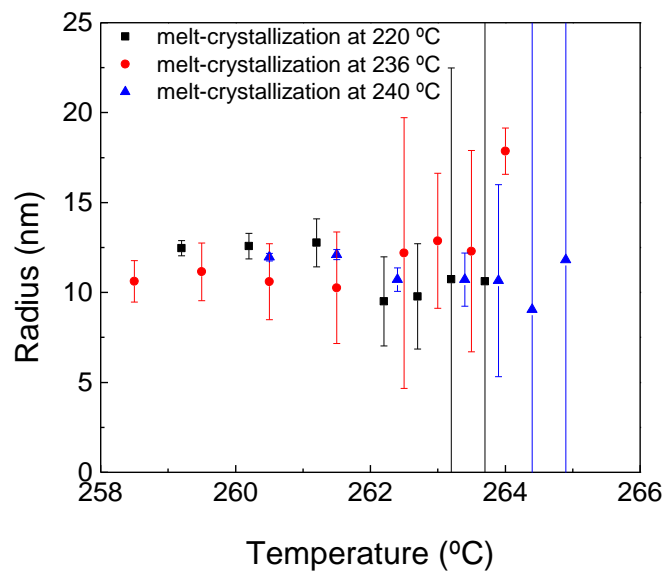

(b) $\mathrm{SPS}-11 \% 3 \mathrm{MS}$ fitting result (thickness)

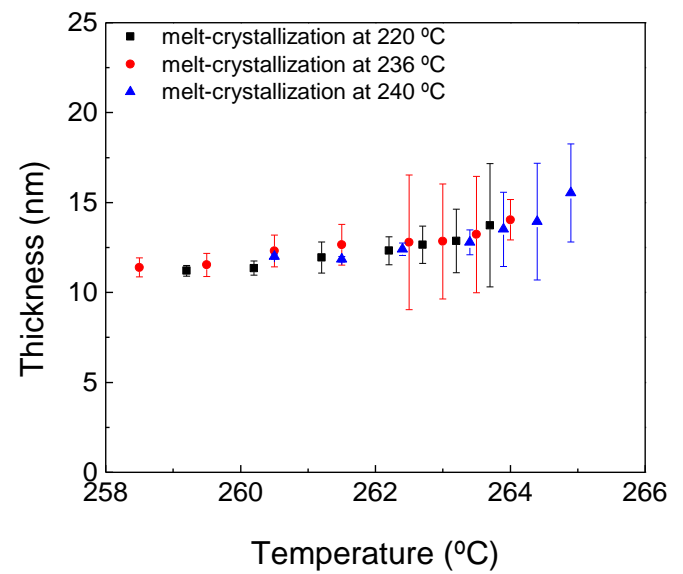

Figure S25. The evolution of (a) radius and (b) thickness of the nanograins during the heating scan which obtained from the reduced SAXS profiles in Figure S24. Based on the fitting parameters, the nanograins are close to disk-like structures.

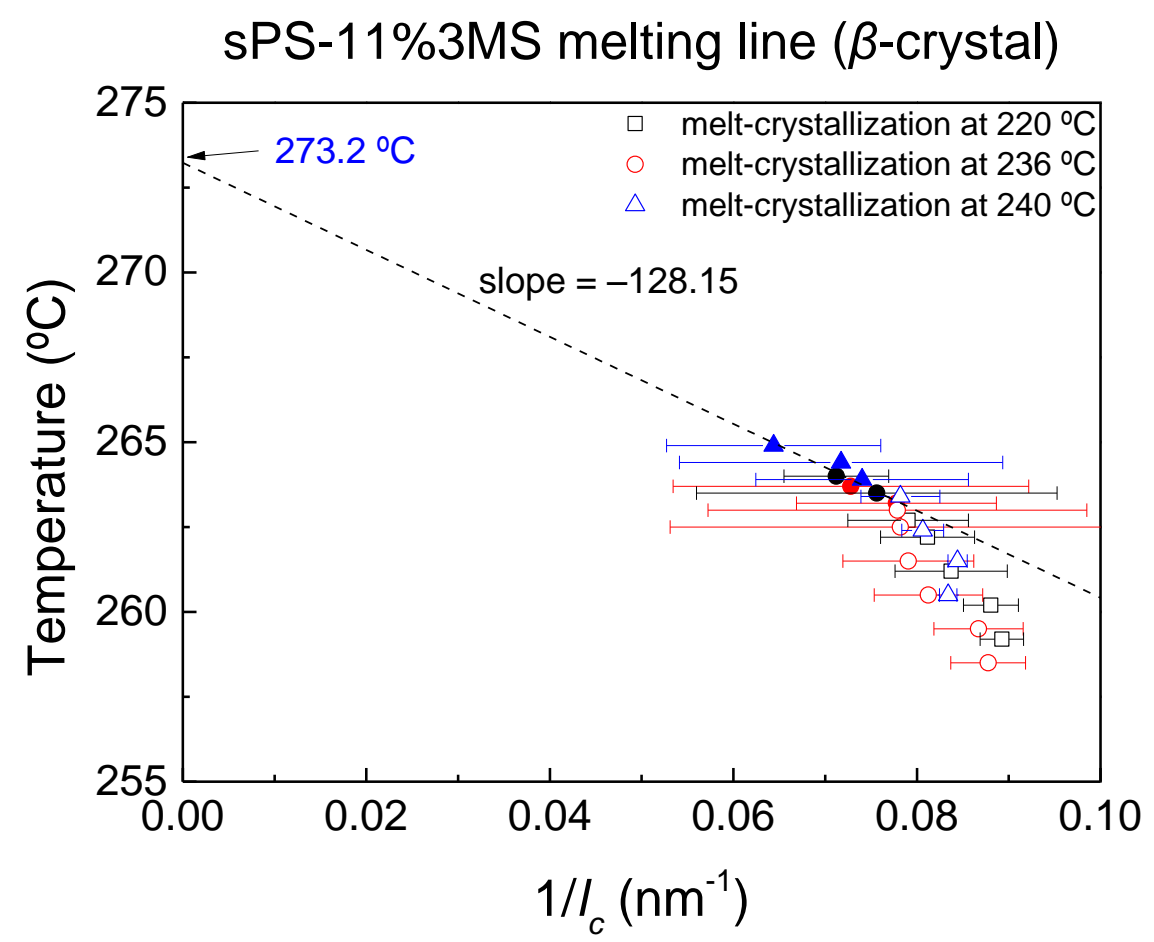

Figure S26. Evolution of the reciprocal crystal thickness $\left(1 / l_{c}\right)$ upon heating for sPS- $11 \% 3 \mathrm{MS}$ meltcrystallized specimens. Dash line represents the melting line that established by extrapolating the data points with solid symbols, giving the equilibrium melting temperature $\left(T_{m}{ }^{0}\right)$ for sPS-11\%3MS $\beta$-crystals as $273.2 \pm 1.3{ }^{\circ} \mathrm{C}$. 
Illustrated in Figure S27 are the simultaneous SAXS/WAXS heating profiles of sPS-2\%4MS cold-crystallized specimens. Corresponding WAXS profiles in Figure $\mathrm{S} 27 \mathrm{~d}-\mathrm{f}$ show that specimens were all formed $\alpha$-dominated crystals. Upon heating, the SAXS profiles basically have the same tendency as the profiles of sPS-3MS random copolymers that the lamellar peak gradually became broader and shifted to the lower $q$-position, which represents the increase of long-period and weakening of the spatial correlation between nanograins; in WAXS profiles, the intensity of reflection peaks gradually decrease without appearing any new reflection peak. Thus, the SAXS/WAXS profiles correspond to the melting behaviors of cold-crystallized sPS- $\% 4 \mathrm{MS}$ specimens. The lamellar peak in the SAXS profiles and the crystalline reflection peaks in the WAXS profiles disappeared after reaching ca. $280^{\circ} \mathrm{C}$. 


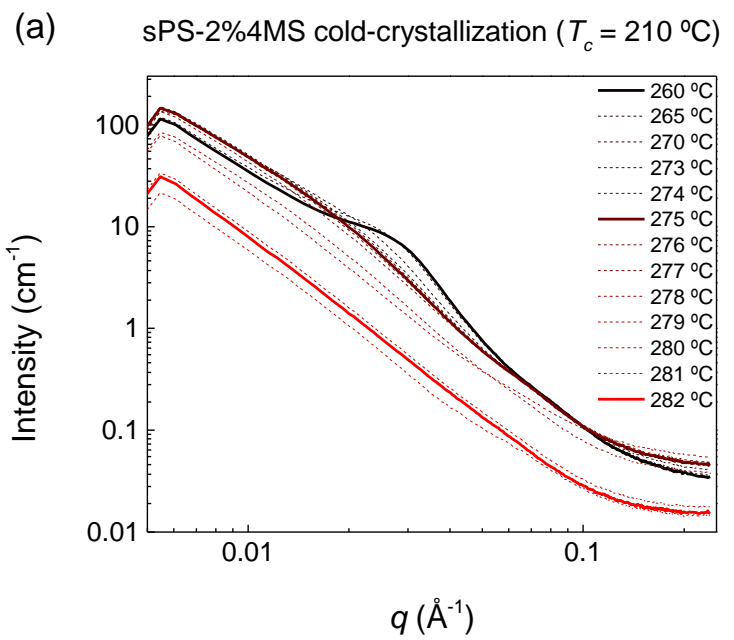

(b) $\quad$ sPS-2\%4MS cold-crystallization $\left(T_{c}=240 \stackrel{\circ}{\circ}\right)$

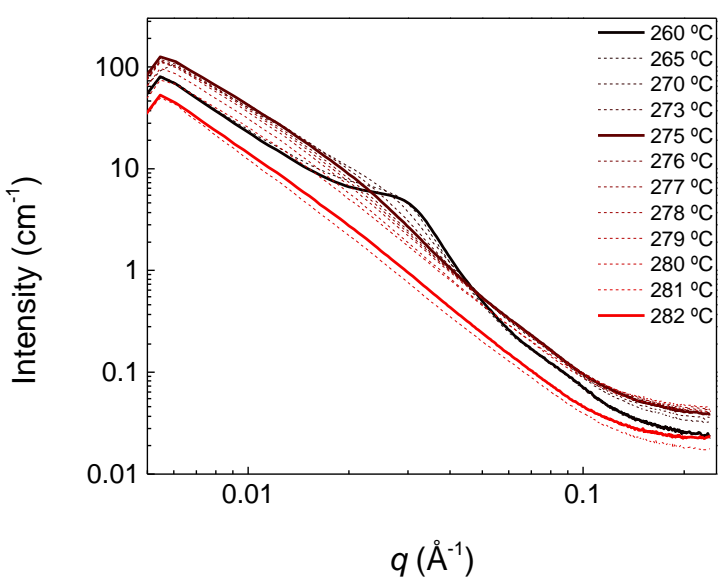

(c) $\quad$ SPS-2\%4MS cold-crystallization $\left(T_{c}=250 \stackrel{\circ}{\circ}\right)$

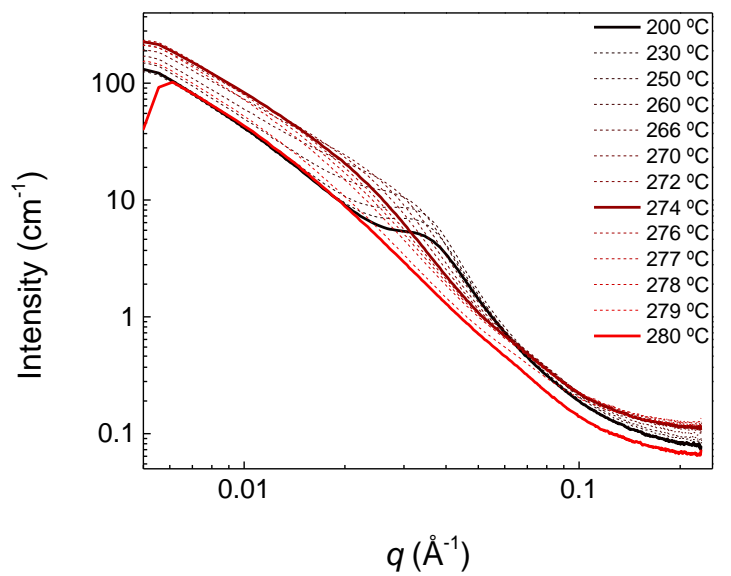

(d) $\quad$ sPS-2\%4MS cold-crystallized $\left(T_{c}=210^{\circ} \mathrm{C}\right)$

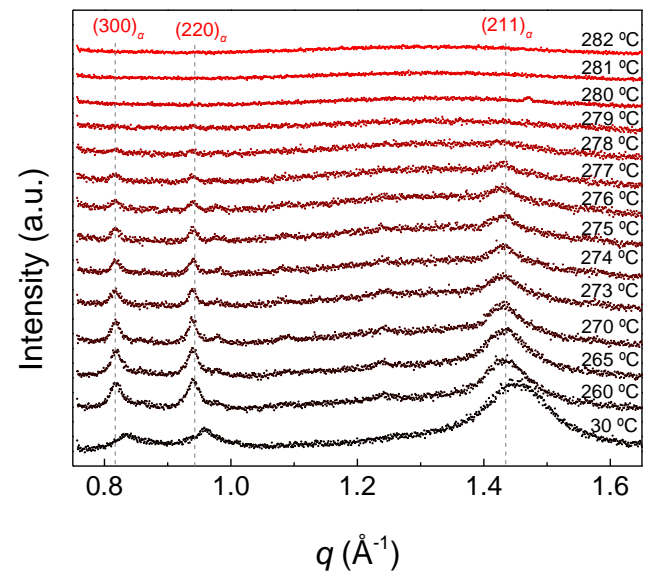

(e) $\quad$ SPS- $2 \% 4 \mathrm{MS}$ cold-crystallized $\left(T_{c}=240 \stackrel{\circ}{\circ}\right)$

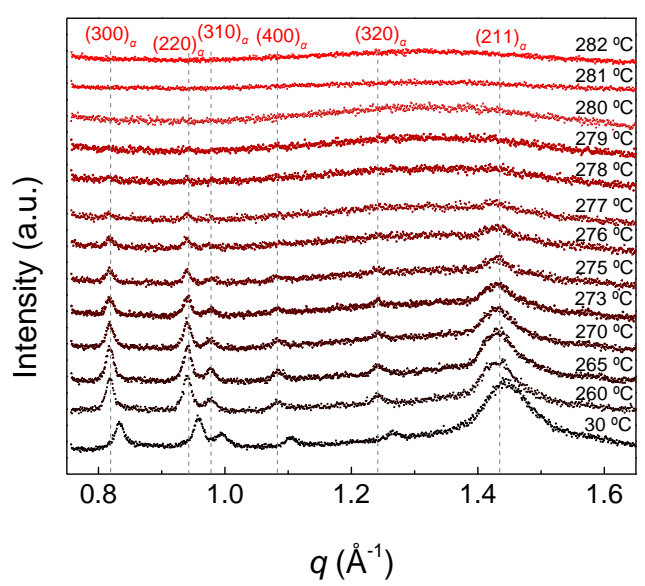

(f) $\quad$ sPS-2\%4MS cold-crystallized $\left(T_{c}=250 \stackrel{\circ}{\circ}\right)$

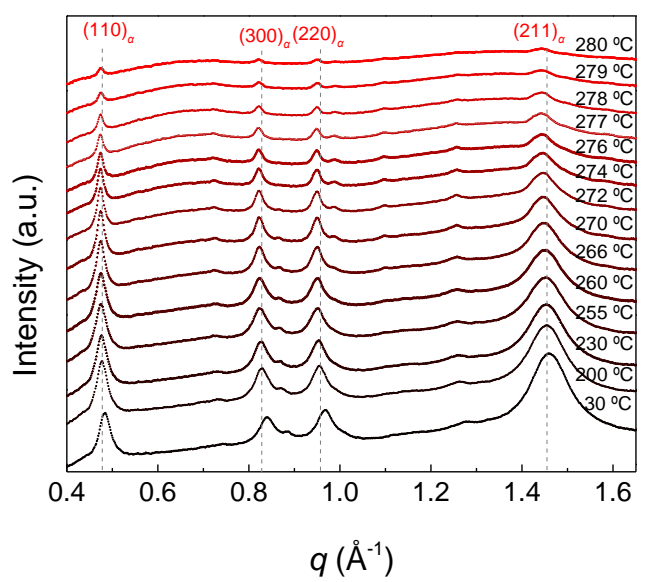

Figure S27. Simultaneous (a)-(c) SAXS and (d)-(f) WAXS heating profiles of sPS-2\%4MS coldcrystallized specimens ( $\alpha$-dominated crystals). The WAXS profiles show that the reflections peaks gradually decrease and completely melt after reaching ca. $280{ }^{\circ} \mathrm{C}$.

After subtracting the molten state scattering profiles, the reduced sPS-2\%4MS coldcrystallized SAXS heating profiles that approached melting are shown in Figure S28. The open symbols are the experimental profiles, and the solid lines are the fitting profiles that the cylindershaped form factor with polydisperse radius model was used. The corresponding fitting parameters of the radius and thickness are presented in Figure S29, and the relation between reciprocal crystal 
thickness $\left(l_{c}^{-1}\right)$ and the experimental temperature is illustrated in Figure S30. The melting line can be constructed by extrapolating the data points (solid symbol), which is just several degrees below melting, and for the case of $l_{c}{ }^{-1} \sim 0$ gives the equilibrium melting temperature $\left(T_{m}{ }^{0}\right)$ of sPS-2\%4MS $\alpha$-crystal as $291.0 \pm 2.3^{\circ} \mathrm{C}$. Based on the depression level of $T_{m}{ }^{0}$ and the slope of the melting line, the penalty energy $(\varepsilon)$ and the fold surface energy $\left(\sigma_{e}\right)$ can be derived via Sanchez-Eby and GibbsThomson theory, which is $\varepsilon=21.7 \pm 16.6 \mathrm{MJ} \mathrm{m}^{-3}$ and $\sigma_{e}=14.9 \pm 2.5 \mathrm{~mJ} \mathrm{~m}^{-2}$.
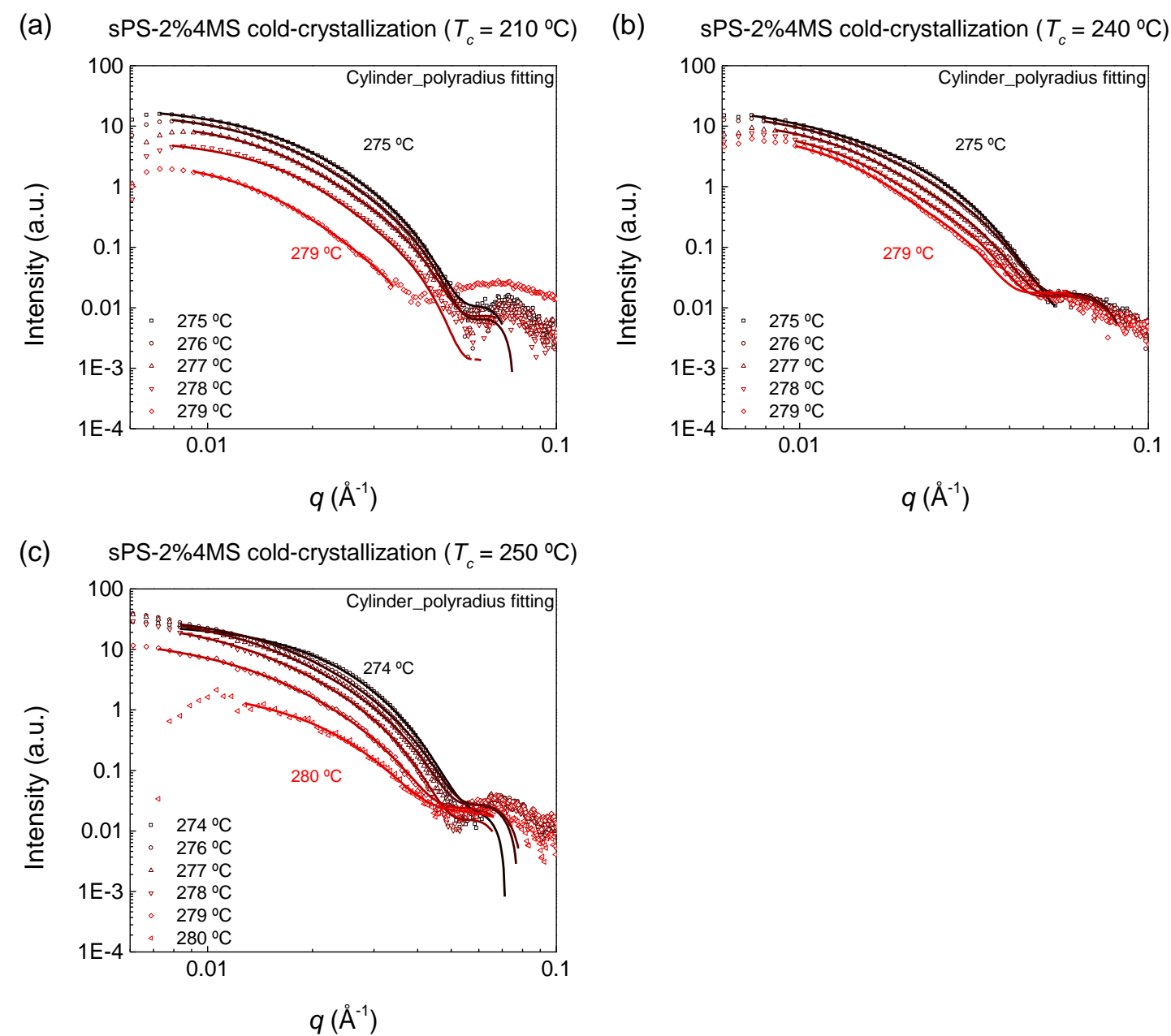

Figure S28. Reduced SAXS heating profiles of sPS-2\%4MS cold-crystallized specimens at $T_{c}=(\mathrm{a})$ 210 , (b) 240 , and (c) $250{ }^{\circ} \mathrm{C}$ before melting (ca. $275-280^{\circ} \mathrm{C}$ ), which gives basically form factor scattering profiles. Solid lines are the fitting profiles by using the cylinder with polydisperse radius form factor model. 
(a) $\quad$ SPS-2\%4MS fitting result (raduis)

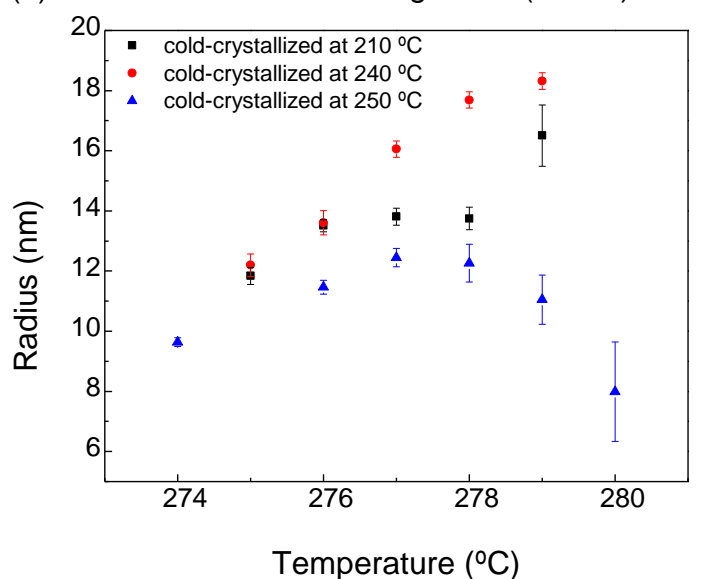

(b) sPS-2\%4MS fitting result (thickness)

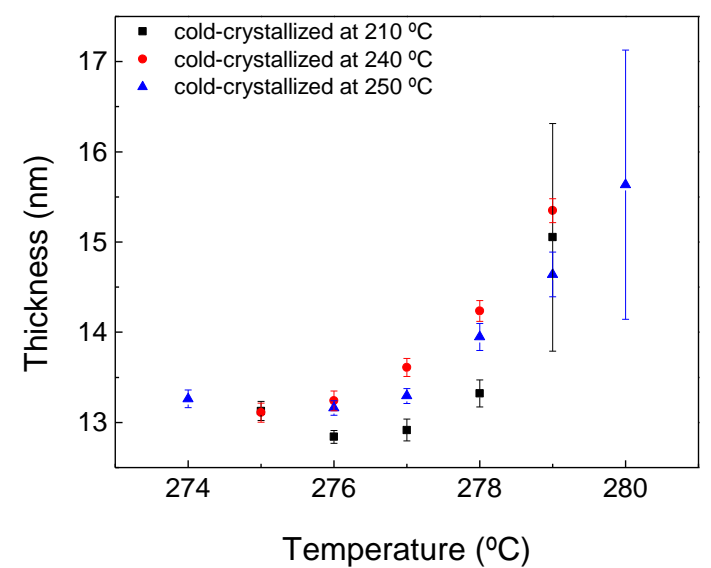

Figure S29. Evolution of (a) radius and (b) thickness of the nanograins during the slow heating scan which obtained from the reduced SAXS profiles in Figure S28. Based on the fitting parameters, the nanograins are close to disk-like structures.

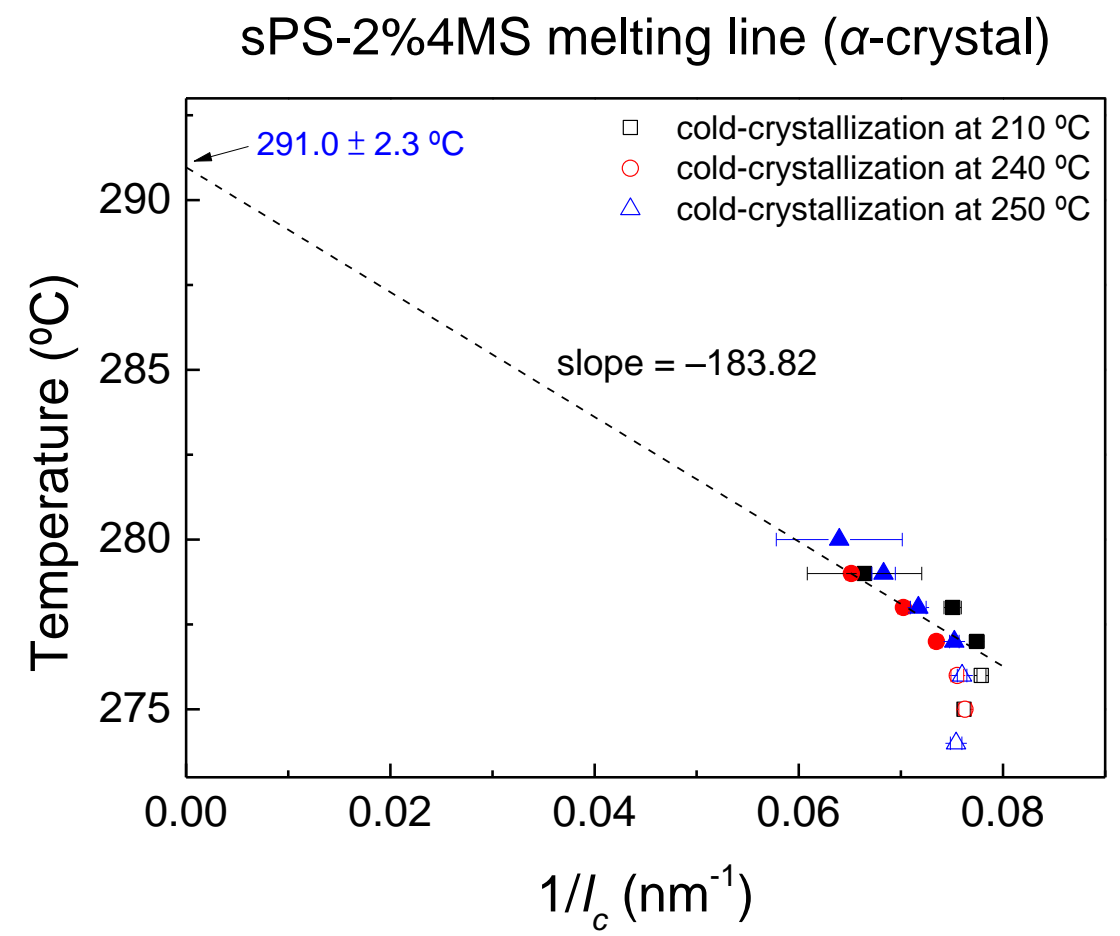

Figure S30. Evolution of the reciprocal crystal thickens $\left(1 / l_{c}\right)$ upon heating for sPS-2\%4MS coldcrystallized specimens. Dash line represents the melting line that established by extrapolating the data points with solid symbols, giving the equilibrium melting temperature $\left(T_{m}{ }^{0}\right)$ for sPS-2\%4MS cold-crystallized $\alpha$-crystals as $291.0 \pm 2.3^{\circ} \mathrm{C}$. 
Illustrated in Figure S31 are the simultaneous SAXS/WAXS heating profiles of sPS-5\%4MS melt-crystallized specimens. Corresponding WAXS profiles in Figure $S 31 \mathrm{f}-\mathrm{j}$ show that specimens were all formed $\alpha$-dominated crystals. Upon heating, the SAXS profiles basically have the same tendency as the profiles of sPS-3MS random copolymers that the lamellar peak gradually became broader and shifted to the lower $q$-position, which represents the increase of long-period and weakening of the spatial correlation between nanograins; in WAXS profiles, the intensity of reflection peaks gradually decrease without appearing any new reflection peak. Thus, the SAXS/WAXS profiles correspond to the melting of melt-crystallized sPS-5\%4MS specimens. Finally, the lamellar peak in the SAXS profiles and the crystalline reflection peaks in the WAXS profiles disappeared after reaching ca. $272{ }^{\circ} \mathrm{C}$. 
(a) $\quad$ sPS-5\%4MS melt-crystallization $\left(T_{c}=195^{\circ} \mathrm{C}\right)$

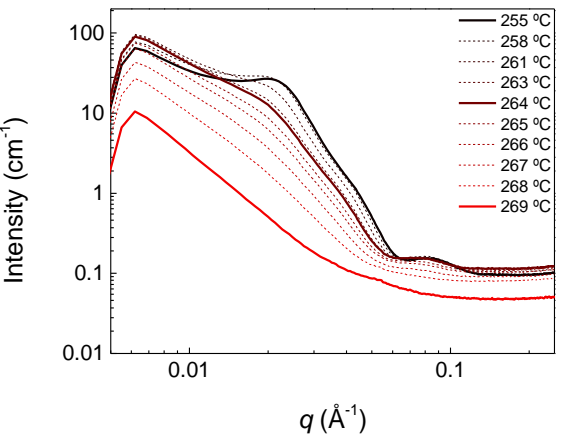

(b) $\quad$ sPS-5\%4MS melt-crystallization $\left(T_{c}=200{ }^{\circ} \mathrm{C}\right)$

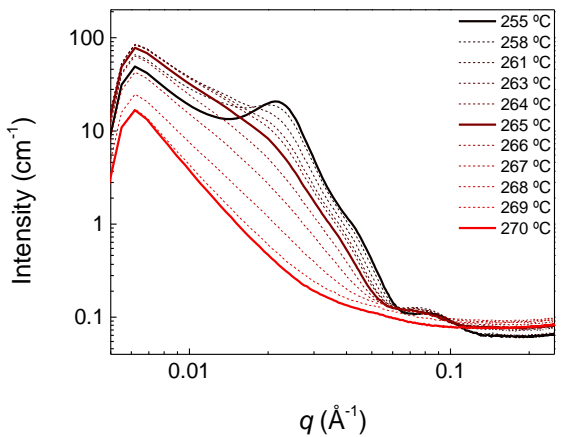

(c) $\quad$ SPS- $5 \% 4 \mathrm{MS}$ melt-crystallization $\left(T_{c}=210^{\circ} \mathrm{C}\right)$

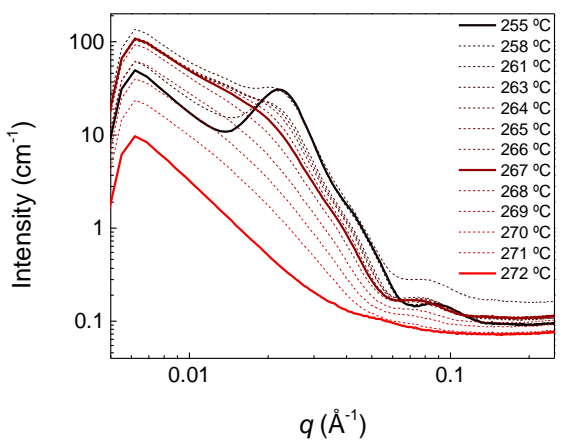

(d) $\quad$ sPS-5\%4MS melt-crystallization $\left(T_{c}=220^{\circ} \mathrm{C}\right)$

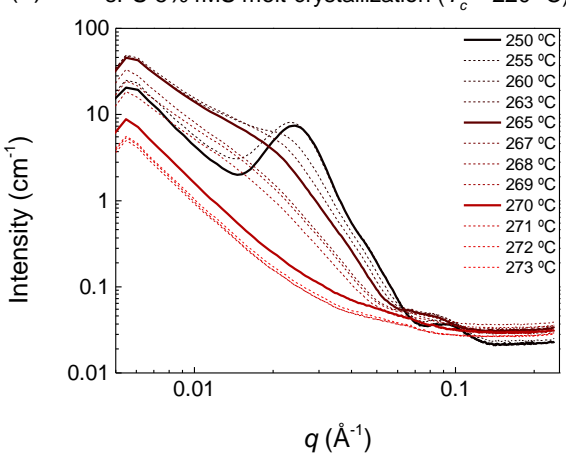

(e) $\quad$ sPS-5\%4MS melt-crystallization $\left(T_{c}=230^{\circ} \mathrm{C}\right)$

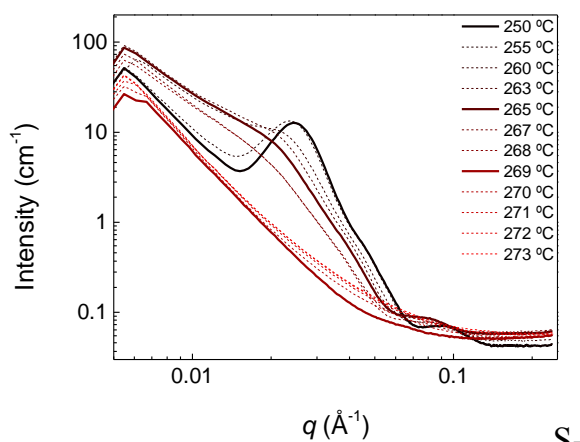

(f) $\mathrm{sPS}-5 \% 4 \mathrm{MS}$ melt-crystallized $\left(T_{c}=195^{\circ} \mathrm{C}\right)$

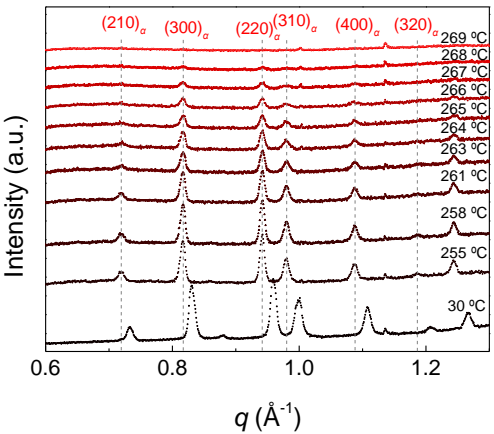

(g) sPS-5\%4MS melt-crystallized $\left(T_{c}=200^{\circ} \mathrm{C}\right)$

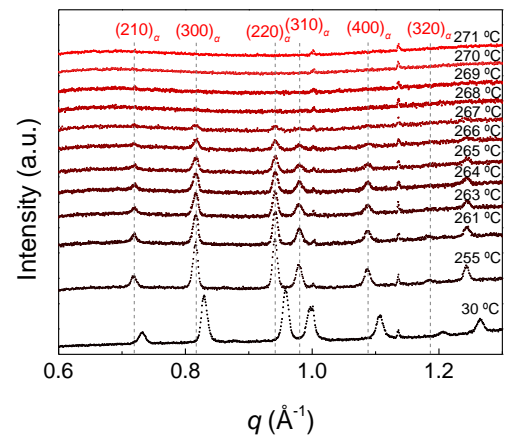

(h) sPS-5\%4MS melt-crystallized $\left(T_{c}=210^{\circ} \mathrm{C}\right)$

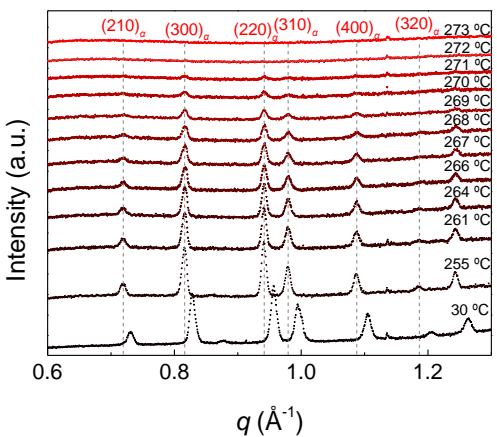

(i) $\mathrm{sPS}-5 \% 4 \mathrm{MS}$ melt-crystallized $\left(T_{c}=220^{\circ} \mathrm{C}\right)$

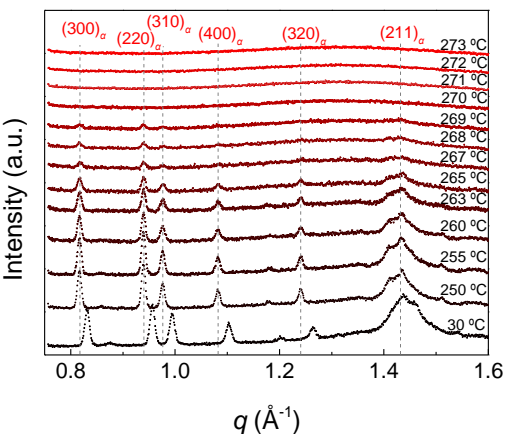

(j) sPS-5\%4MS melt-crystallized $\left(T_{c}=230{ }^{\circ} \mathrm{C}\right)$

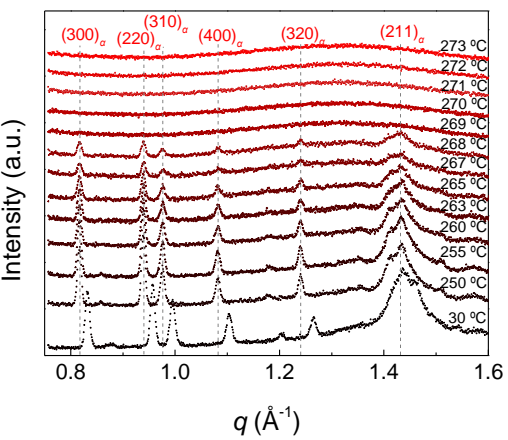


Figure S31. Simultaneous (a)-(e) SAXS and (f)-(j) WAXS heating profiles of sPS-5\%4MS meltcrystallized specimens ( $\alpha$-dominated crystals). The WAXS profiles show that the reflections peaks gradually decrease and completely melt after reaching ca. $272{ }^{\circ} \mathrm{C}$.

After subtracting the molten state scattering profiles, the reduced sPS-5\%4MS meltcrystallized SAXS heating profiles that approached melting are shown in Figure S32. The open symbols are the experimental profiles, and the solid lines are the fitting profiles that the cylindershaped form factor with polydisperse radius model was used. The corresponding fitting parameters of the radius and thickness are presented in Figure S33, and the relation between reciprocal crystal thickness $\left(l_{c}{ }^{-1}\right)$ and the experimental temperature is illustrated in Figure S34. The melting line can be constructed by extrapolating the data points (solid symbol), which is just several degrees below melting, and for the case of $l_{c}{ }^{-1} \sim 0$ gives the equilibrium melting temperature $\left(T_{m}{ }^{0}\right)$ of sPS-5\%4MS $\alpha$-crystal as $286.3 \pm 5.6^{\circ} \mathrm{C}$. Based on the depression level of $T_{m}{ }^{0}$ and the slope of the melting line, the penalty energy $(\varepsilon)$ and the fold surface energy $\left(\sigma_{e}\right)$ can be derived via Sanchez-Eby and GibbsThomson theory, which is $\varepsilon=22.3 \pm 16.2 \mathrm{MJ} \mathrm{m}^{-3}$ and $\sigma_{e}=21.2 \pm 7.1 \mathrm{~mJ} \mathrm{~m}^{-2}$. 
(a) sPS-5\%4MS melt-crystallization $\left(T_{c}=195^{\circ} \mathrm{C}\right)$

(b) sPS-5\%4MS melt-crystallization $\left(T_{c}=200 \stackrel{\circ}{\circ}\right)$
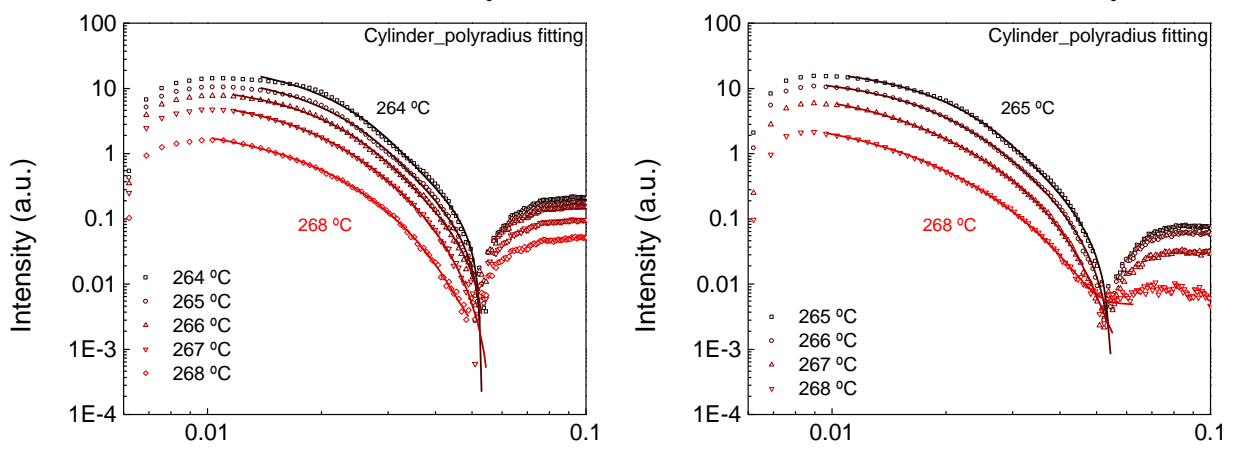

$q\left(\AA^{-1}\right)$

$q\left(\AA^{-1}\right)$

(c) sPS-5\%4MS melt-crystallization $\left(T_{c}=210^{\circ} \mathrm{C}\right)$

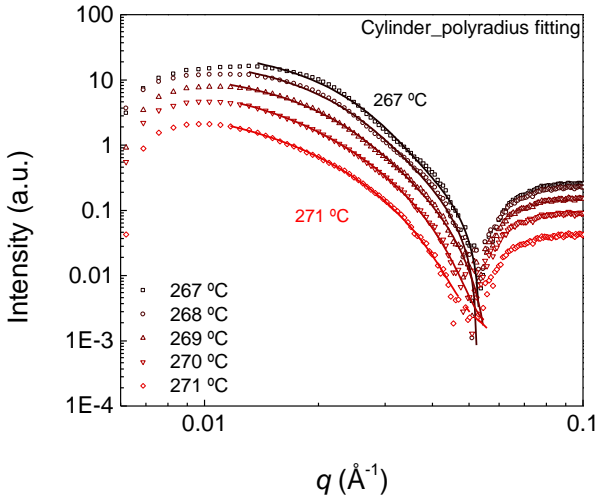

(d) $\quad$ PPS-5\%4MS melt-crystallization $\left(T_{c}=220 \stackrel{\circ}{\circ}\right)$

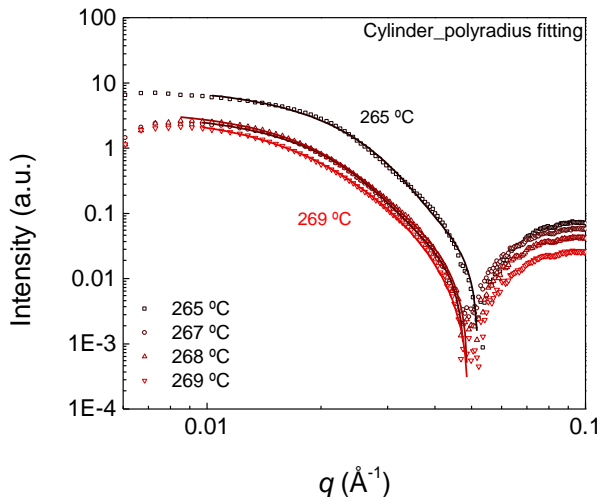

(e) $\quad$ SPS- $5 \% 4 \mathrm{MS}$ melt-crystallization $\left(T_{c}=230 \stackrel{\circ}{\circ} \mathrm{C}\right)$

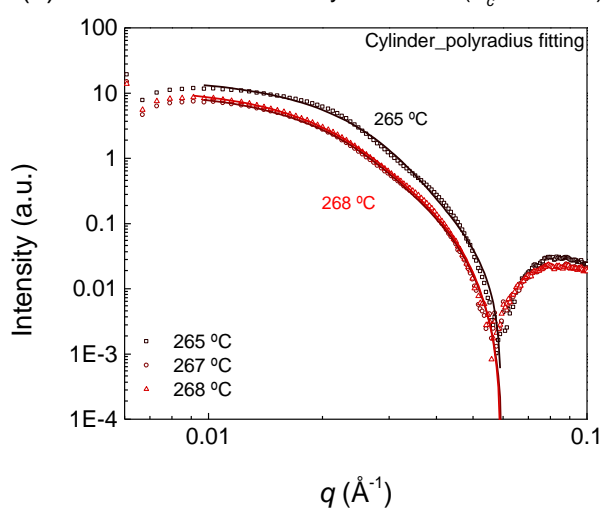

Figure S32. Reduced SAXS heating profiles of sPS-5\%4MS cold-crystallized specimens at $T_{c}=$ (a) 195, (b) 200, (c) 210, (d) 220, and (e) $230^{\circ} \mathrm{C}$ before melting (ca. $264-271^{\circ} \mathrm{C}$ ), which gives basically form factor scattering profiles. Solid lines are the fitting profiles by using the cylinder with polydisperse radius form factor model. 

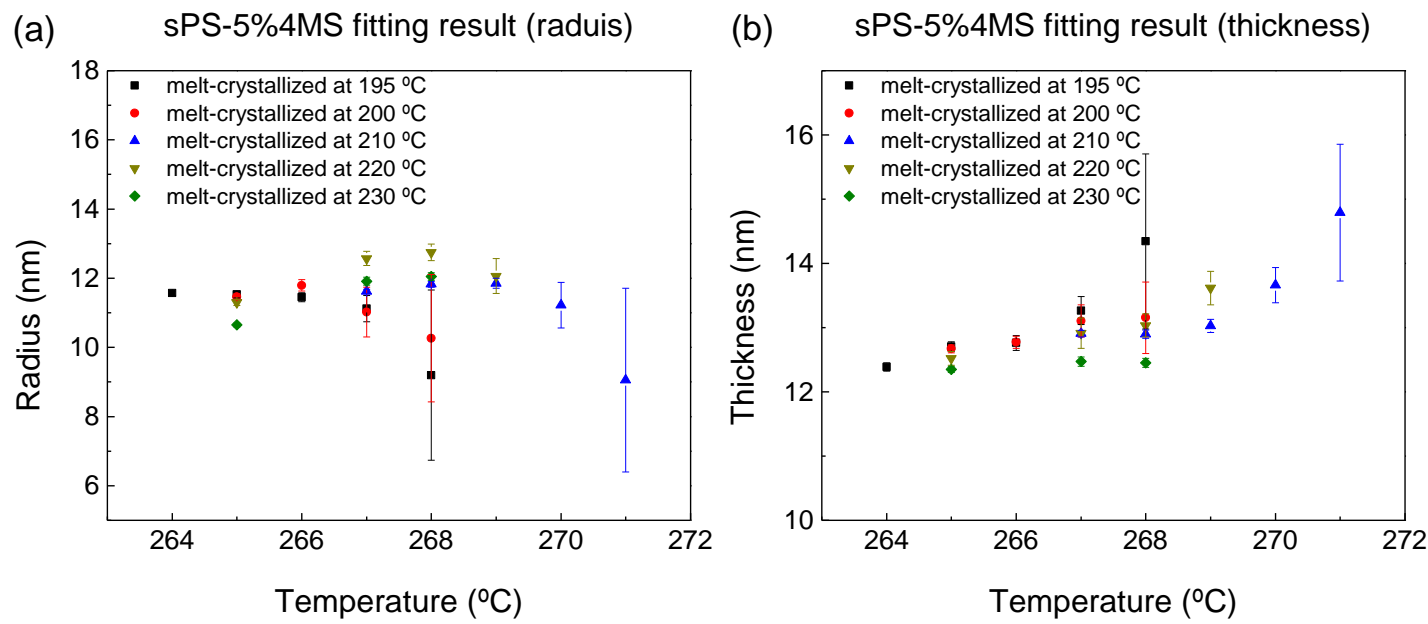

Figure S33. Evolution of (a) radius and (b) thickness of the nanograins during the slow heating scan which obtained from the reduced SAXS profiles in Figure S32. Based on the fitting parameters, the nanograins are close to disk-like structures.

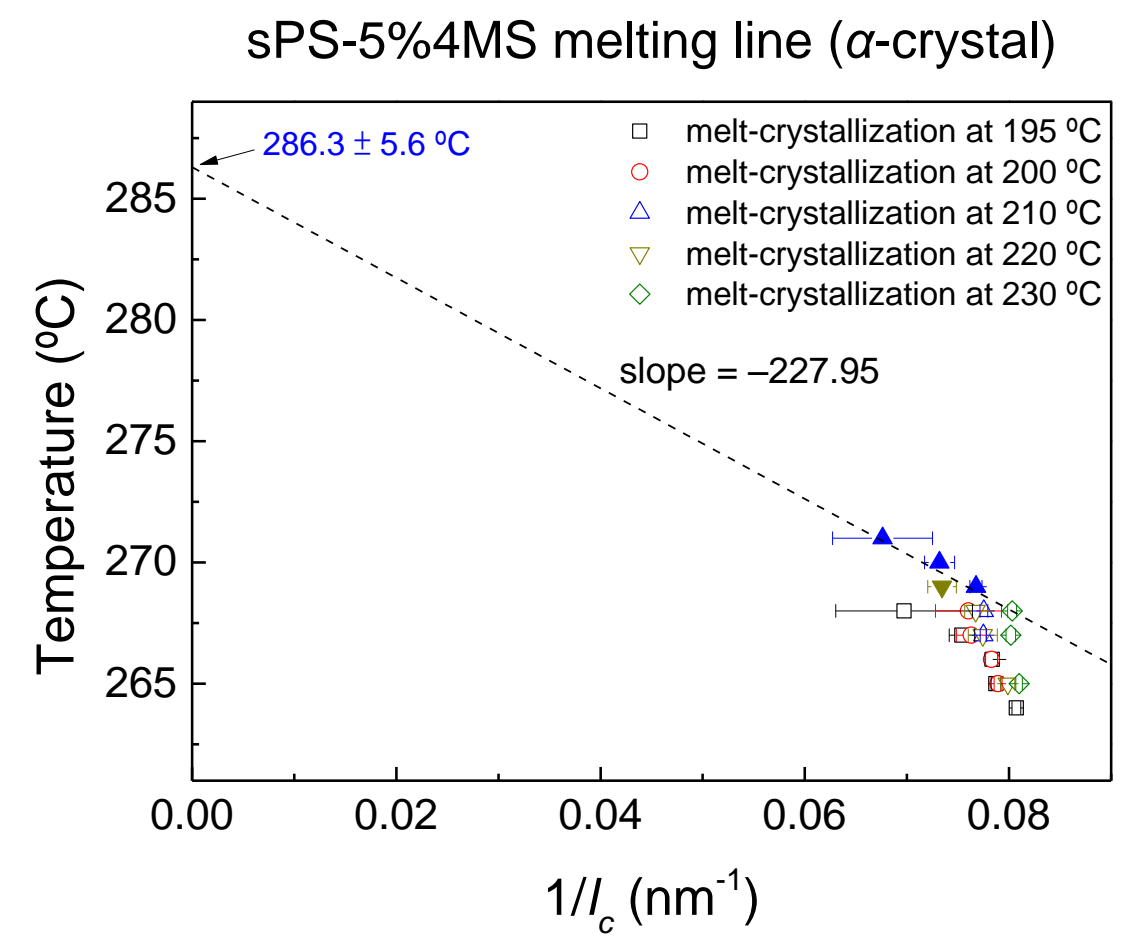

Figure S34. Evolution of the reciprocal crystal thickens $\left(1 / l_{c}\right)$ upon heating for sPS-5\%4MS meltcrystallized specimens. Dash line represents the melting line that established by extrapolating the data points with solid symbols, giving the equilibrium melting temperature $\left(T_{m}{ }^{0}\right)$ for sPS-5\%4MS $\alpha$ crystals as $286.3 \pm 5.6^{\circ} \mathrm{C}$. 
Illustrated in Figure S35 are the simultaneous SAXS/WAXS heating profiles of sPS-10\%4MS melt-crystallized specimens. Corresponding WAXS profiles in Figure $\mathrm{S} 35 \mathrm{~d}-\mathrm{f}$ show that specimens were all formed $\alpha$-dominated crystals. Upon heating, compared with previous cases, the evolutions of SAXS profiles did not have obvious change in $q_{c}$ position, and the scattering became weaker until melting; in WAXS profiles, the intensity of reflection peaks gradually decrease without appearing any new reflection peak. Thus, the SAXS/WAXS profiles correspond to the melting of melt-crystallized sPS-10\%4MS specimens. Finally, the lamellar peak in the SAXS profiles and the crystalline reflection peaks in the WAXS profiles disappeared after reaching ca. $259{ }^{\circ} \mathrm{C}$. 
(a) $\quad$ SPS-10\%4MS melt-crystallization $\left(T_{c}=195 \stackrel{\circ}{\circ}\right)$

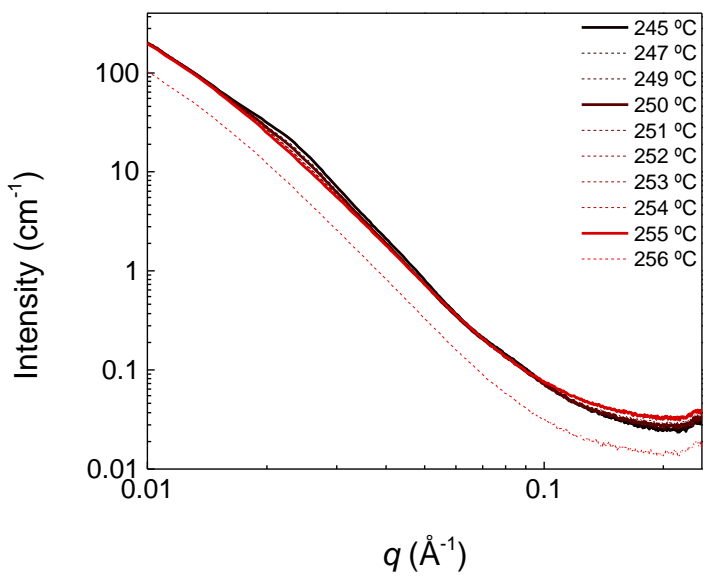

(b) $\quad$ sPS-10\%4MS melt-crystallization $\left(T_{c}=200 \stackrel{\circ}{\circ}\right)$

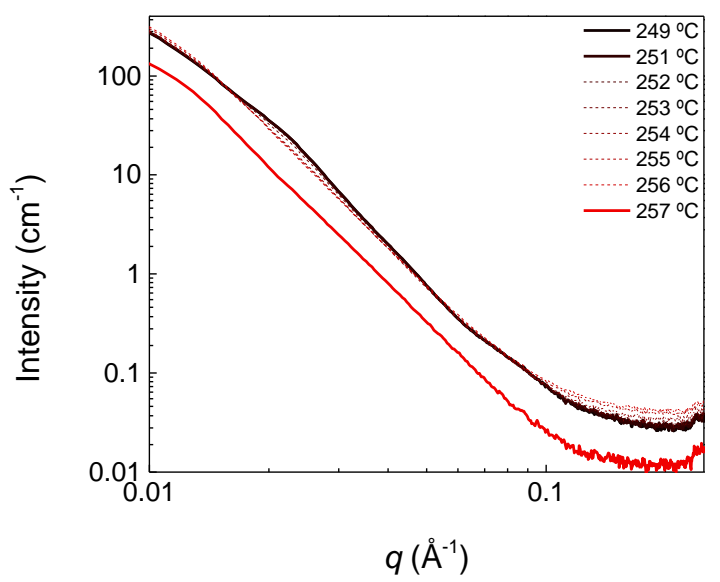

(c) $\quad$ SPS-10\%4MS melt-crystallization $\left(T_{c}=205^{\circ} \mathrm{C}\right)$

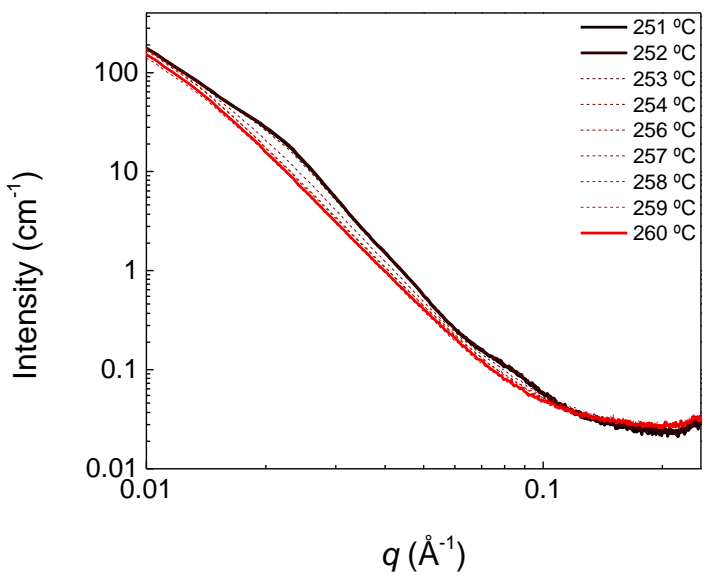

(d) sPS-10\%4MS melt-crystallized $\left(T_{c}=195^{\circ} \mathrm{C}\right)$

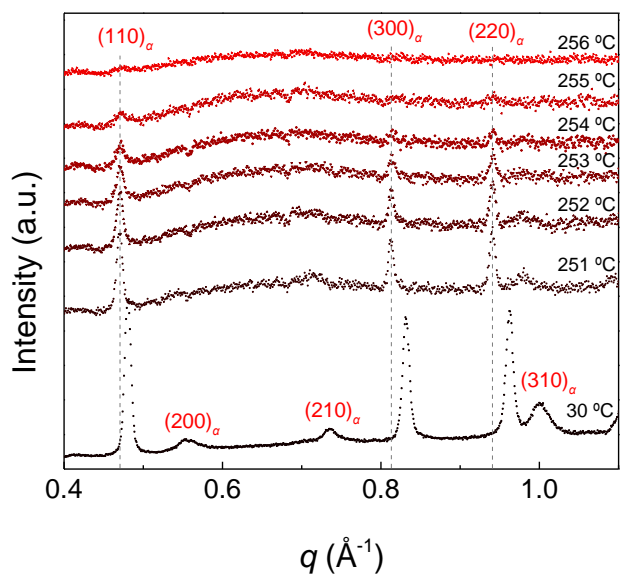

(e) $\quad$ sPS-10\%4MS melt-crystallized $\left(T_{c}=200 \stackrel{\circ}{C}\right)$

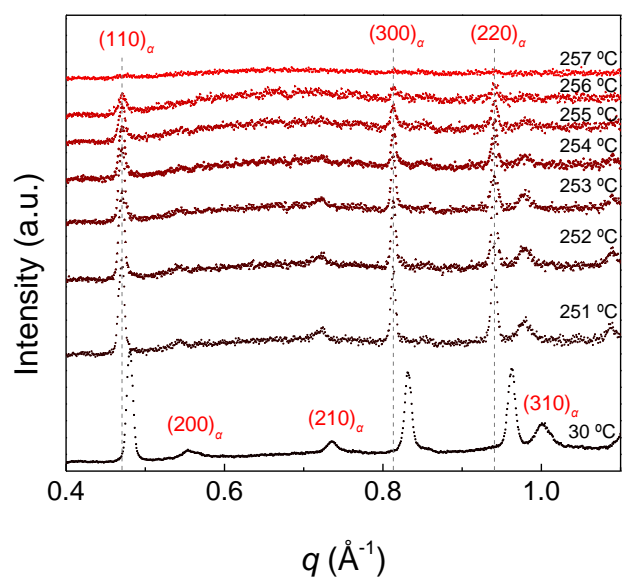

(f) sPS-10\%4MS melt-crystallized $\left(T_{c}=205^{\circ} \mathrm{C}\right)$

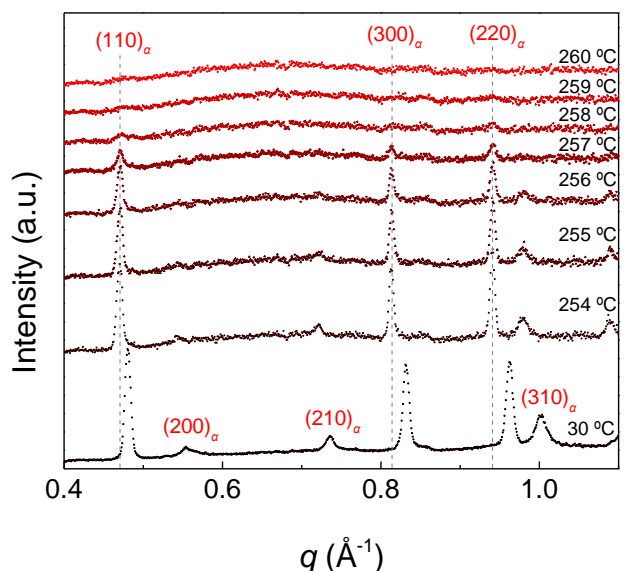

Figure S35. Simultaneous (a)-(e) SAXS and (f)-(j) WAXS heating profiles of sPS-10\%4MS meltcrystallized specimens ( $\alpha$-dominated crystals). The WAXS profiles show that the reflections peaks gradually decrease and completely melt after reaching ca. $259^{\circ} \mathrm{C}$.

After subtracting the molten state scattering profiles, the reduced sPS-10\%4MS meltcrystallized SAXS heating profiles that approached melting are shown in Figure S36. The open symbols are the experimental profiles, and the solid lines are the fitting profiles that the cylindershaped form factor with polydisperse radius model was used. The corresponding fitting parameters of the radius and thickness are presented in Figure S37, and the relation between reciprocal crystal 
thickness $\left(l_{c}^{-1}\right)$ and the experimental temperature is illustrated in Figure S38. The melting line can be constructed by extrapolating the data points (solid symbol) which is just several degrees below melting. Due to the data points for melt-crystallized at $205^{\circ} \mathrm{C}$ specimen are deviated from others, the melting line was constructed depending on the results of specimens that $T_{c}=195$ and $200{ }^{\circ} \mathrm{C}$, and for the case of $l_{c}{ }^{-1} \sim 0$ gives the equilibrium melting temperature $\left(T_{m}{ }^{9}\right)$ of sPS-10\%4MS $\alpha$ crystal as $274.4 \pm 5.7^{\circ} \mathrm{C}$. Based on the depression level of $T_{m}{ }^{0}$ and the slope of the melting line, the penalty energy $(\varepsilon)$ and the fold surface energy $\left(\sigma_{e}\right)$ can be derived via Sanchez-Eby and GibbsThomson theory, which is $\varepsilon=28.3 \pm 8.2 \mathrm{MJ} \mathrm{m}^{-3}$ and $\sigma_{e}=28.8 \pm 7.8 \mathrm{~mJ} \mathrm{~m}^{-2}$.
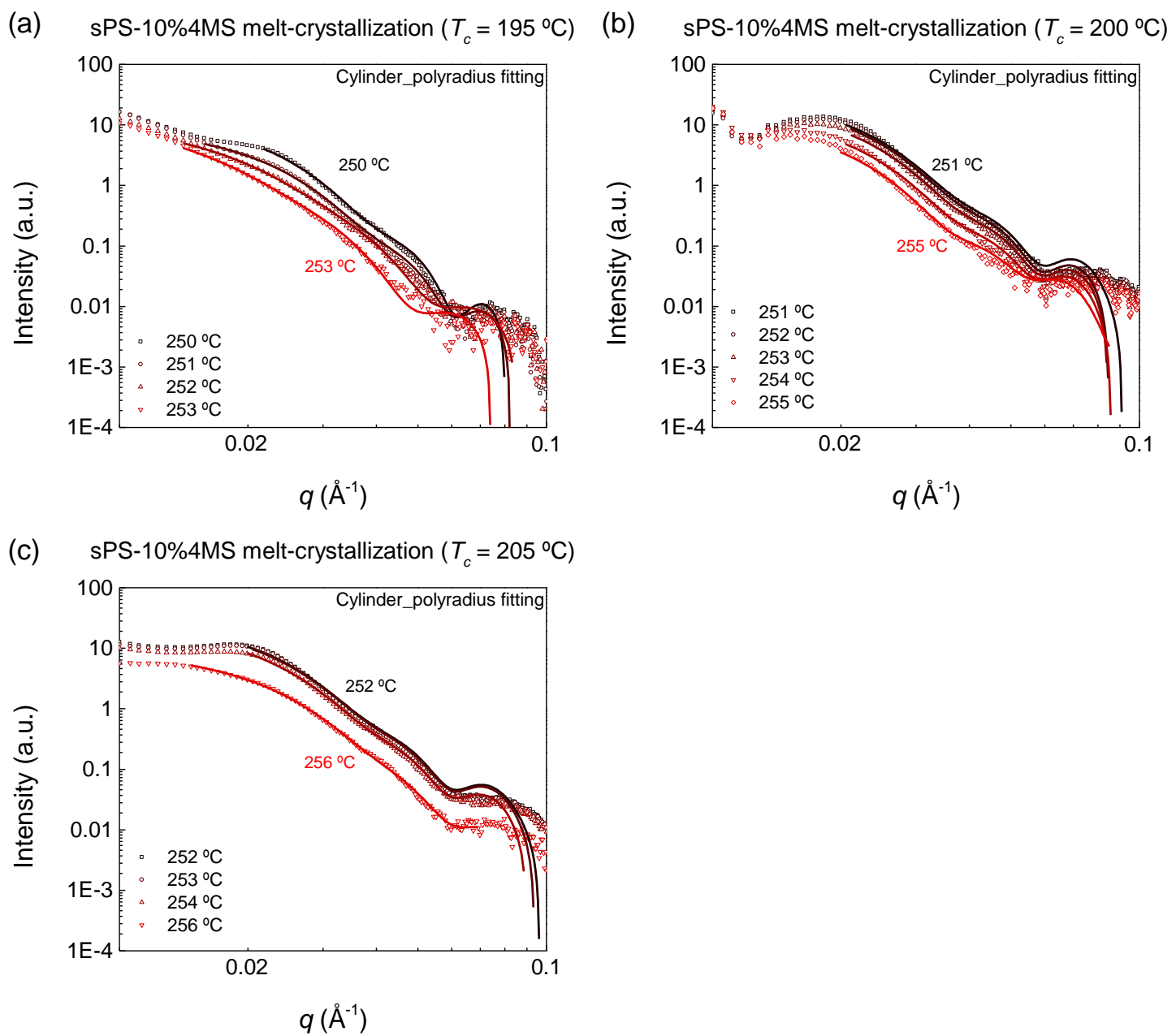

Figure S36. Reduced SAXS heating profiles of sPS-5\%4MS cold-crystallized specimens at $T_{c}=(\mathrm{a})$ 195 , (b) 200 , and (c) $205^{\circ} \mathrm{C}$ before melting (ca. $250-256^{\circ} \mathrm{C}$ ), which gives basically form factor scattering profiles. Solid lines are the fitting profiles by using the cylinder with polydisperse radius form factor model. 
(a) SPS-10\%4MS fitting result (raduis)

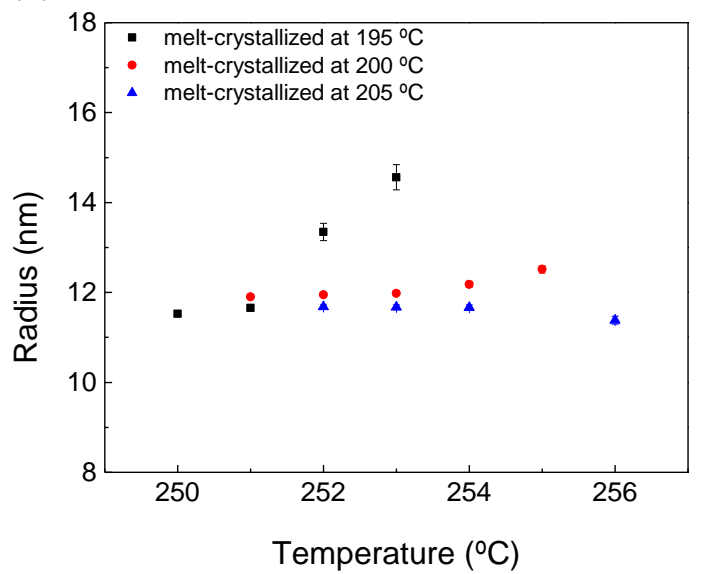

(b) sPS-10\%4MS fitting result (thickness)

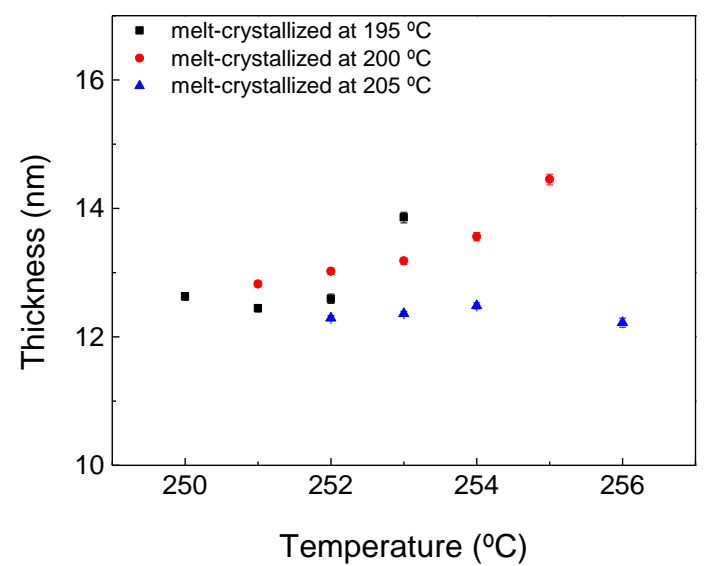

Figure S37. Evolution of (a) radius and (b) thickness of the nanograins during the slow heating scan which obtained from the reduced SAXS profiles in Figure S36. Based on the fitting parameters, the nanograins are close to disk-like structures.

sPS-10\%4MS melting line ( $\alpha$-crystal)

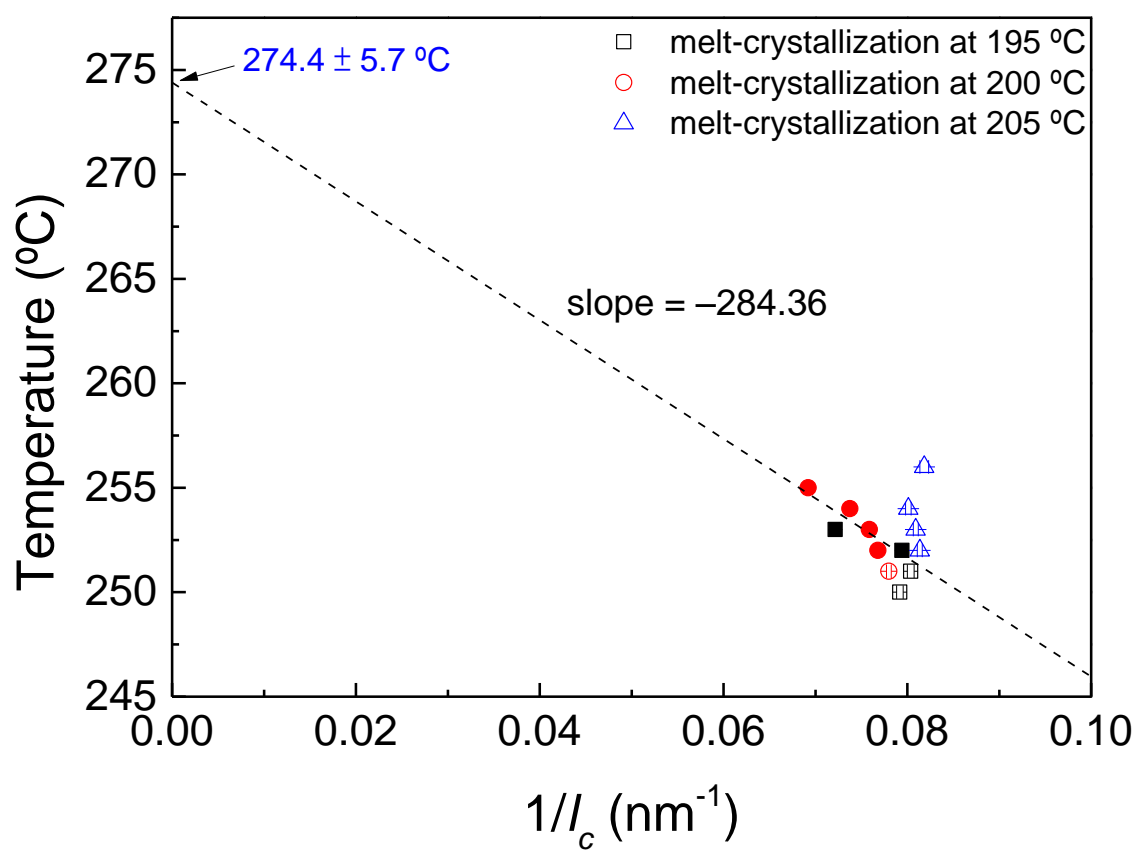

Figure S38. Evolution of the reciprocal crystal thickens $\left(1 / l_{c}\right)$ upon heating for sPS- $10 \% 4 \mathrm{MS}$ meltcrystallized specimens. Dash line represents the melting line that established by extrapolating the data points with solid symbols, giving the equilibrium melting temperature $\left(T_{m}{ }^{0}\right)$ for sPS-10\%4MS $\alpha$-crystals as $274.4 \pm 5.7^{\circ} \mathrm{C}$. 
As mention in the previous report ${ }^{2}$, only 2 mol $\%$ 4MS content can form $\beta$-crystals in sPS4MS systems, showing that the 4MS unit has stronger suppression on $\beta$-crystal formation. The simultaneous SAXS/WAXS heating profiles for sPS-2\%4MS melt-crystallized specimens are illustrated in Figure S39. Corresponding WAXS profiles in Figure S39c-d show that the meltcrystallized at $T_{c}=220^{\circ} \mathrm{C}$ specimen gave $\beta$-dominated crystals; however, the specimen that meltcrystallized at $T_{c}=220^{\circ} \mathrm{C}$ gave $\alpha$-dominated crystals. Upon heating, the SAXS profiles basically have the same tendency as the profiles of sPS-3MS random copolymers that the lamellar peak gradually became broader and shifted to the lower $q$-position, which represents the increase of longperiod and weakening of the spatial correlation between nanograins; in WAXS profiles, the intensity of reflection peaks gradually decreases without appearing any new reflection peak. Thus, the SAXS/WAXS profiles correspond to the melting behaviors of melt-crystallized sPS-2\%4MS specimens.

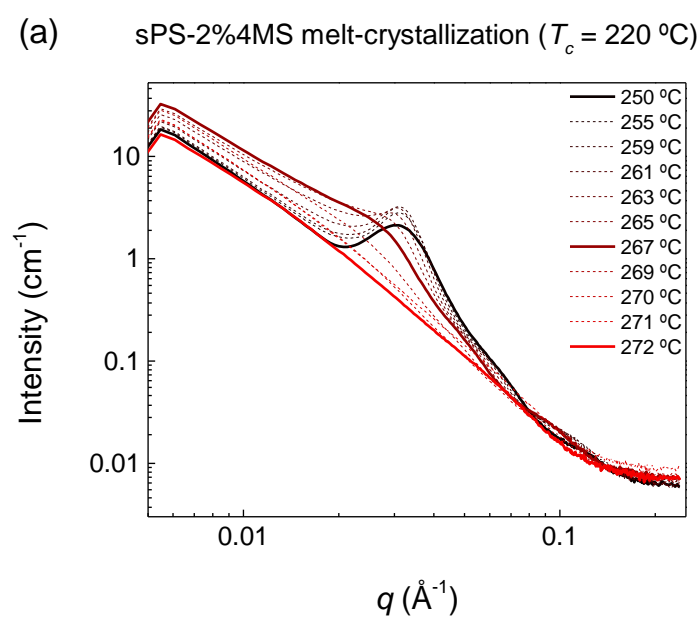

(c) sPS-2\%4MS melt-crystallized $\left(T_{c}=220 \stackrel{\circ}{\circ}\right)$
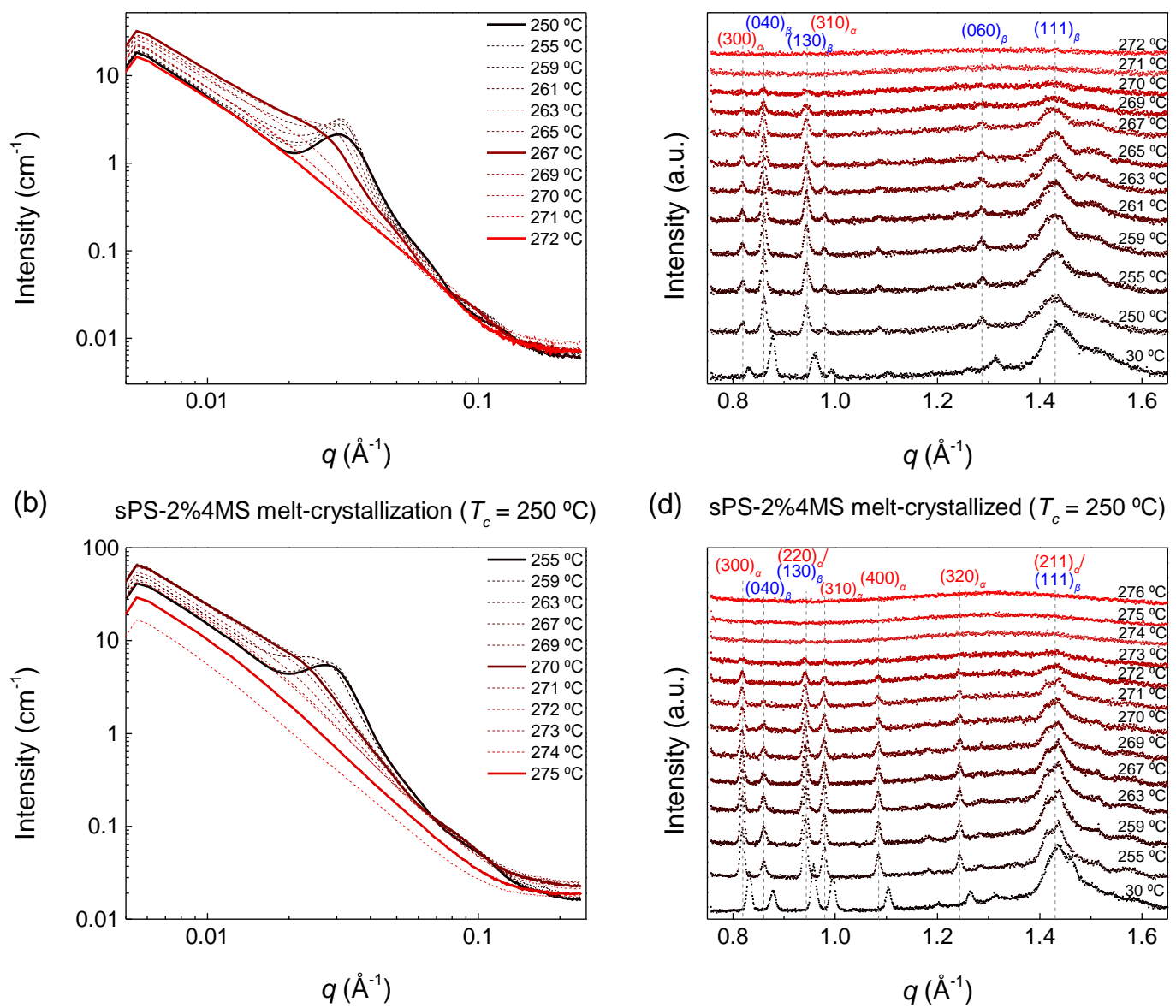

(d) sPS-2\%4MS melt-crystallized $\left(T_{c}=250 \stackrel{\circ}{\circ}\right)$

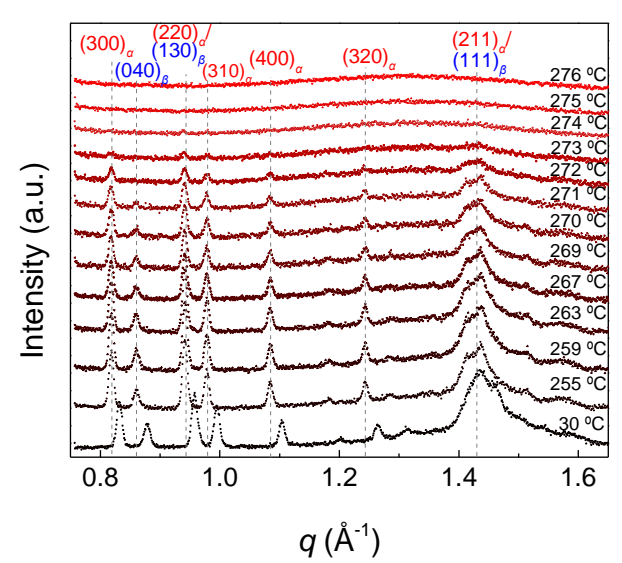

Figure S39. Simultaneous (a)-(b) SAXS and (c)-(d) WAXS heating profiles of sPS-2\%4MS meltcrystallized specimens $(\beta$-dominated crystals). To be noted, that from the WAXS profiles, specimen with $T_{c}=220^{\circ} \mathrm{C}$ gave $\beta$-dominated crystals but gave $\alpha$-dominated crystals for specimen with $T_{c}=$ $250^{\circ} \mathrm{C}$. 
After subtracting the molten state scattering profiles, the reduced sPS-2\%4MS meltcrystallized SAXS heating profiles that approached melting are shown in Figure S40. The open symbols are the experimental profiles, and the solid lines are the fitting profiles that the cylindershaped form factor with polydisperse radius model was used. The corresponding fitting parameters of the radius and thickness are presented in Figure S41, and the relation between reciprocal crystal thickness $\left(l_{c}{ }^{-1}\right)$ and the experimental temperature is illustrated in Figure S42. Although the specimen with $T_{c}=250{ }^{\circ} \mathrm{C}$ gave $\alpha$-dominated structure, the data points that approach melting stayed almost at the same line (solid symbols in Figure S42). Thus, the melting line was constructed by these data points, and for the case of $l_{c}{ }^{-1} \sim 0$ gives the equilibrium melting temperature $\left(T_{m}{ }^{0}\right)$ of sPS-2\%4MS $\beta$-crystal as $295.1 \pm 3.1{ }^{\circ} \mathrm{C}$. Based on the depression level of $T_{m}{ }^{0}$ and the slope of the melting line, the penalty energy $(\varepsilon)$ and the fold surface energy $\left(\sigma_{e}\right)$ can be derived via Sanchez-Eby and Gibbs-Thomson theory, which is $\varepsilon=137.0 \pm 39.5 \mathrm{MJ} \mathrm{m}^{-3}$ and $\sigma_{e}=31.7 \pm 4.0 \mathrm{~mJ} \mathrm{~m}^{-2}$.

(a) SPS-2\%4MS melt-crystallization $\left(T_{c}=220 \stackrel{\circ}{\circ}\right)$

(b) $\quad$ sPS-2\%4MS melt-crystallization $\left(T_{c}=250 \stackrel{\circ}{\circ}\right)$
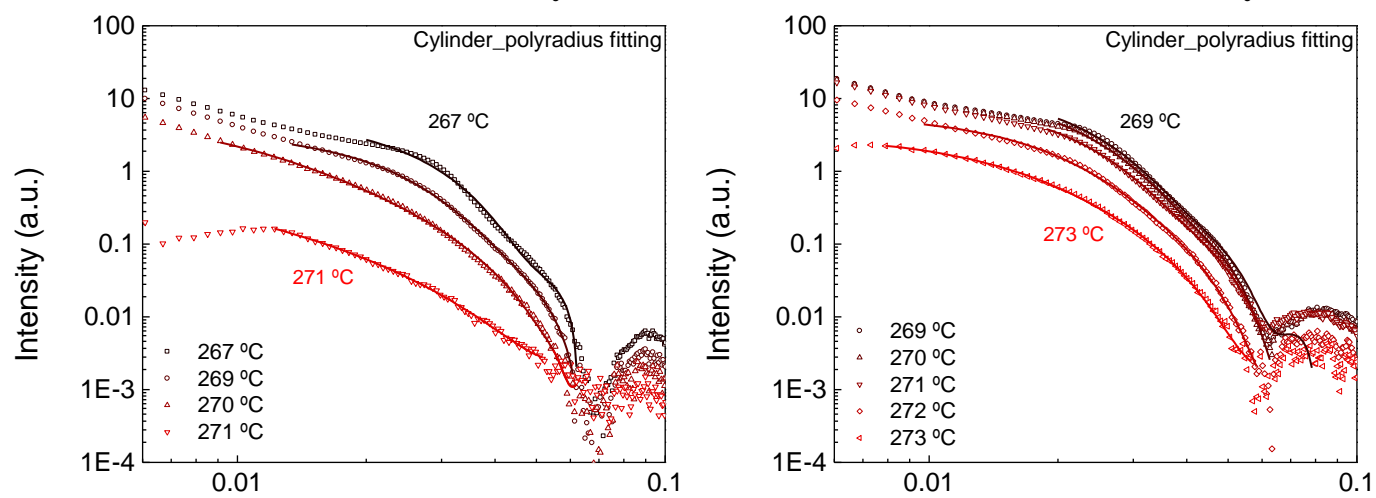

$q\left(\AA^{-1}\right)$

$q\left(\AA^{-1}\right)$

Figure S40. Reduced SAXS heating profiles of sPS-2\%4MS melt-crystallized specimens at $T_{c}=(\mathrm{a})$ 220 , and (b) $250{ }^{\circ} \mathrm{C}$ before melting (ca. $267-273{ }^{\circ} \mathrm{C}$ ), which gives basically form factor scattering profiles. Solid lines are the fitting profiles by using the cylinder with polydisperse radius form factor model.
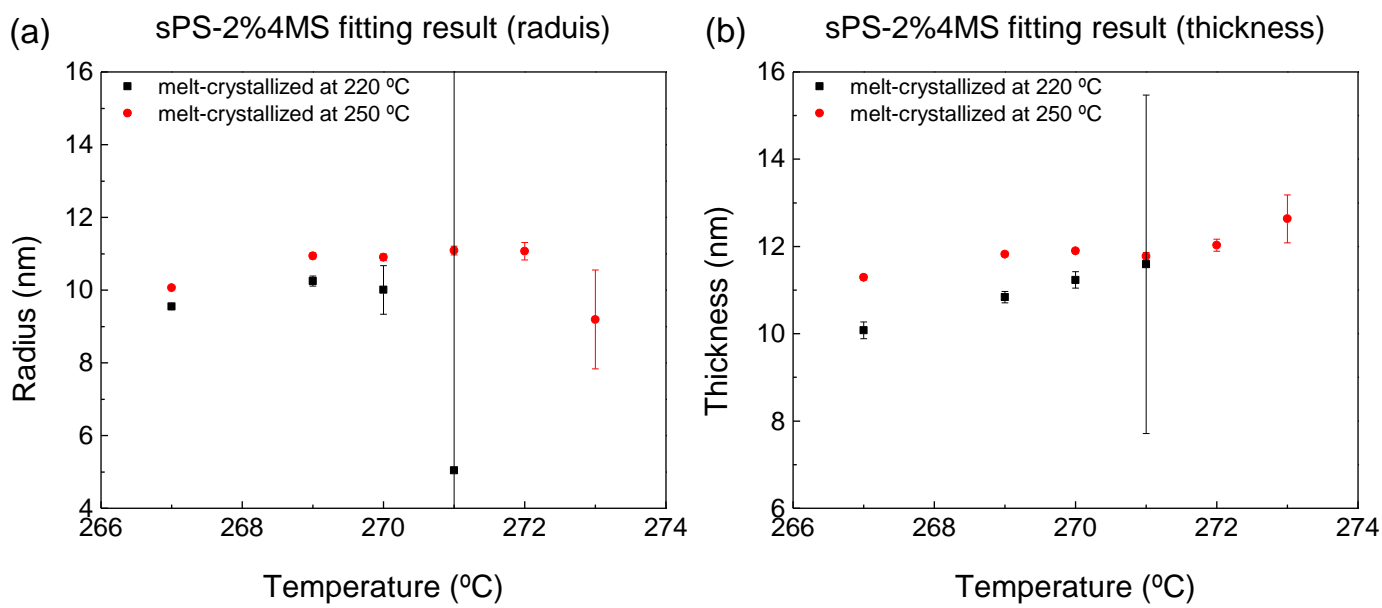

Figure S41. Evolution of (a) radius and (b) thickness of the nanograins during the slow heating scan which obtained from the reduced SAXS profiles in Figure S40. Based on the fitting parameters, the nanograins are close to disk-like structures. 


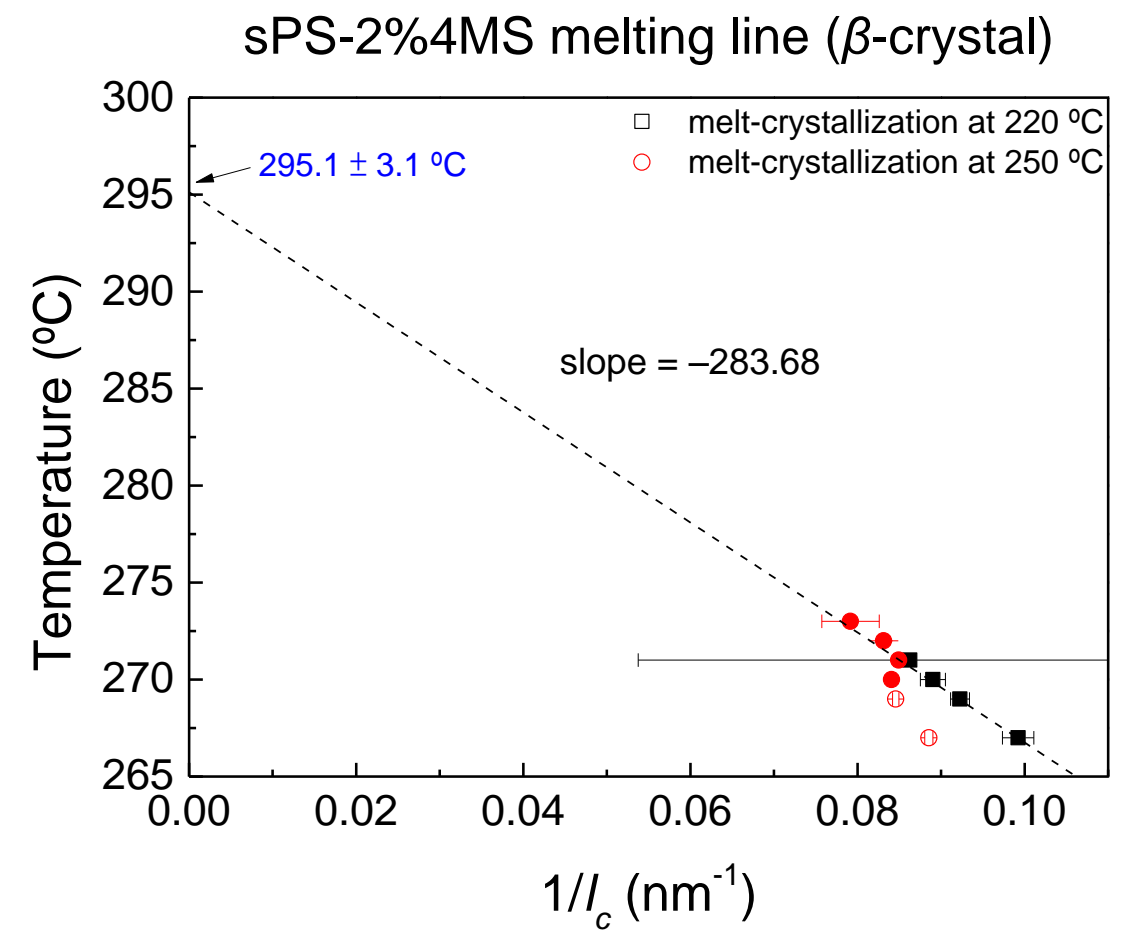

Figure S42. Evolution of the reciprocal crystal thickens $\left(1 / l_{c}\right)$ upon heating for sPS-2\%4MS meltcrystallized specimens. Dash line represents the melting line that established by extrapolating the data points with solid symbols, giving the equilibrium melting temperature $\left(T_{m}{ }^{0}\right)$ for sPS- $2 \% 4 \mathrm{MS} \beta$ crystals as $295.1 \pm 3.1^{\circ} \mathrm{C}$. 


\section{Equilibrium Heat of Fusion $\left(\Delta \mathrm{H}_{m}{ }^{0}\right)$}

Differential scanning calorimeter (DSC) measurements were carried out by using a PerkinElmer Diamond instrument with purging nitrogen gas $(20 \mathrm{~mL} / \mathrm{min})$ at a $10{ }^{\circ} \mathrm{C} / \mathrm{min}$ heating/cooling rate between 30 and $300{ }^{\circ} \mathrm{C}$ temperature range. Before the measurement, the instrument was calibrated with Indium and Zinc standard. The full-ranged Wide-angle X-ray scattering (WAXS) measurements were performed at wiggler beamline BL17A/BL23A of Taiwan Light Source (TLS), National Synchrotron Radiation Research Center (NSRRC).

Presented in Figure S43 are the (a) DSC traces and (b) WAXS profiles of sPS-3MS $\alpha$ crystals, which were prepared via cold-crystallization at different $T_{c}$ and different isothermal periods. The melting point depression phenomenon is apparent in the DSC traces. The WAXS profiles show that all specimens were all $\alpha$-dominated crystals, and, after peak deconvolution, the crystallinity $\left(X_{c}\right)$ can be calculated by the peak area of crystalline peaks over the total area. Compared among the same comonomer contents, the higher $T_{c}$ makes specimen has larger $X_{c}$. Combing the heat of fusion from DSC traces and the corresponding $X_{c}$ from WAXS profiles, the equilibrium heat of fusion of $\alpha$-crystals $\left(\Delta H_{m}{ }^{9}\right)$ can be estimated and the values are $83.8 \pm 9.4 \mathrm{MJ}$ $\mathrm{m}^{-3}$ (sPS-5\%3MS), $68.3 \pm 2.3 \mathrm{MJ} \mathrm{m}^{-3}$ (sPS-11\%3MS) and $73.9 \pm 1.7 \mathrm{MJ} \mathrm{m}^{-3}$ (sPS-21\%3MS).

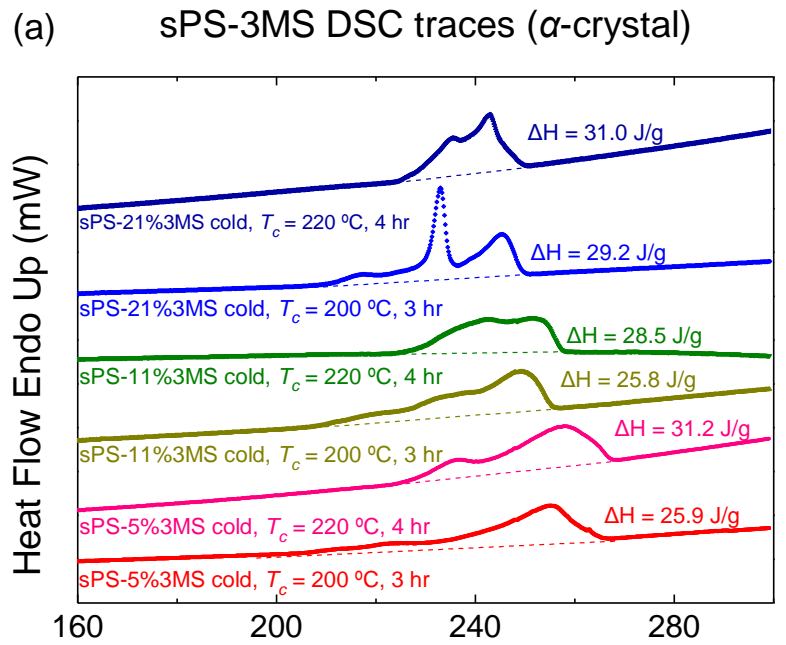

Temperature $\left({ }^{\circ} \mathrm{C}\right)$

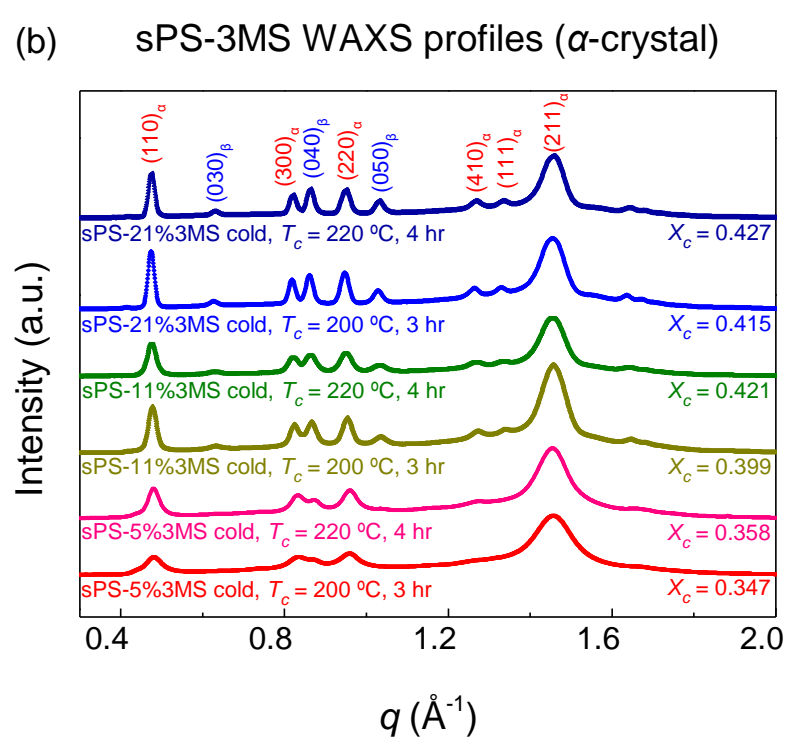

Figure S43. The (a) DSC heating traces (rate: $10^{\circ} \mathrm{C} / \mathrm{min}$ ) and (b) WAXS profiles (collected at room temperature) for cold-crystallized sPS-3MS specimens. The WAXS profiles show that all specimens gave $\alpha$-dominated structure. Based on the heat of fusion and the crystallinity under different crystallization processes, the equilibrium heat of fusion of $\alpha$-crystal $\left(\Delta H_{m}{ }^{0}\right)$ for sPS-3MS copolymers are: $83.8 \pm 9.4 \mathrm{MJ} \mathrm{m}^{-3}$ (sPS-5\%3MS), $68.3 \pm 2.3 \mathrm{MJ} \mathrm{m}^{-3}$ (sPS-11\%3MS) and $73.9 \pm$ $1.7 \mathrm{MJ} \mathrm{m}^{-3}$ (sPS-21\%3MS). 
Figure S44 shown the (a) DSC traces and (b) WAXS profiles of $\beta$-crystals for 5 and $11 \mathrm{~mol} \%$ $3 \mathrm{MS}$ content, which were prepared via melt-crystallization. The WAXS profiles show that all the specimens formed $\beta$-dominated crystals and note that the sPS-21\%3MS specimen can only form $\alpha$ dominated crystals. The $X_{c}$ increase as increasing the $T_{c}$ for sPS-5\%3MS specimens, but invert for sPS-11\%3MS specimens, which might due to the $T_{c}$ approaches the melting temperature that slows down the nucleation/growth process. Combing the heat of fusion from DSC traces and the corresponding $X_{c}$ from WAXS profiles, the equilibrium heat of fusion of $\beta$-crystals $\left(\Delta H_{m}{ }^{0}\right)$ can be estimated and the values are $88.0 \pm 2.6 \mathrm{MJ} \mathrm{m}^{-3}$ (sPS-5\%3MS) and $83.5 \pm 0.0 \mathrm{MJ} \mathrm{m}^{-3}$ (sPS$11 \% 3 \mathrm{MS})$.

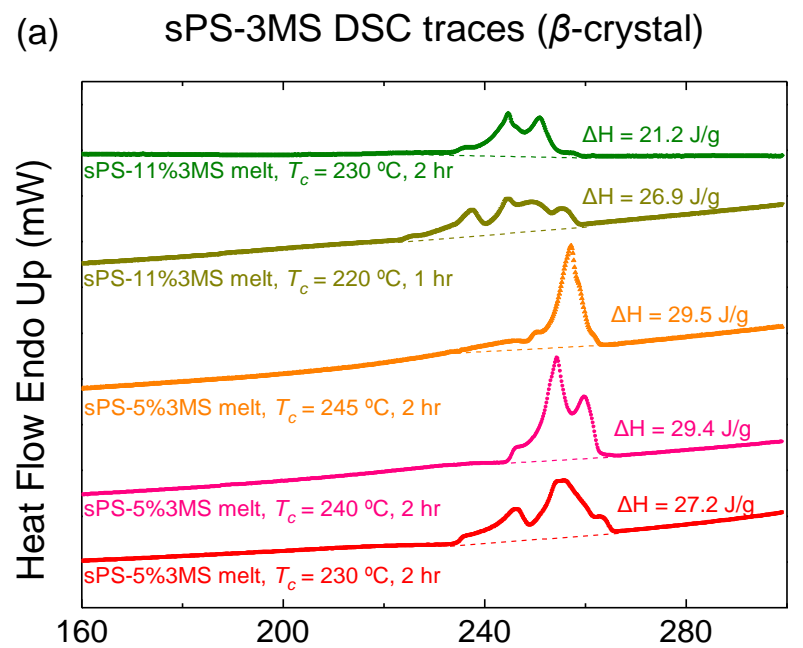

Temperature $\left({ }^{\circ} \mathrm{C}\right)$ (b) sPS-3MS WAXS Profiles ( $\beta$-crystal)

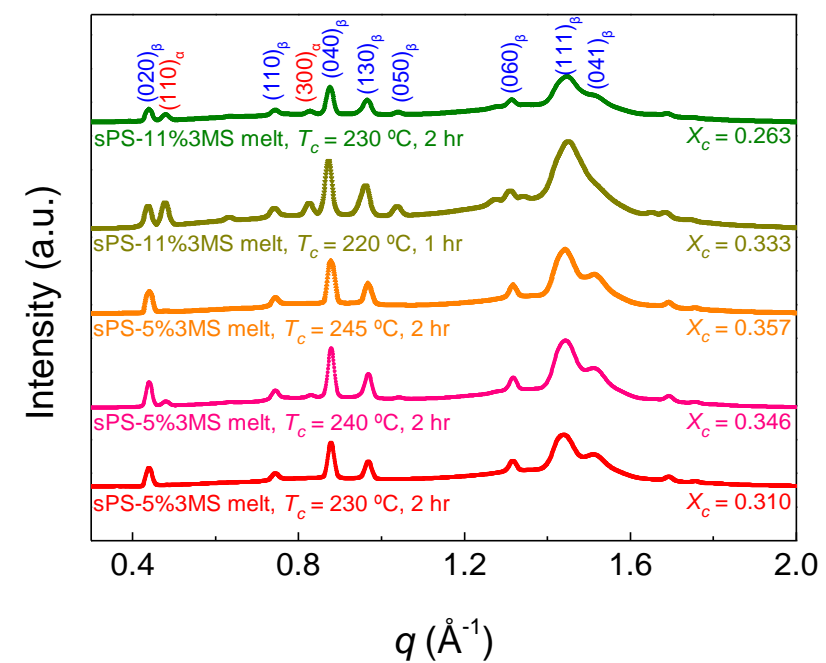

Figure S44. The (a) DSC heating traces (rate: $10^{\circ} \mathrm{C} / \mathrm{min}$ ) and (b) WAXS profiles (collected at room temperature) for melt-crystallized sPS-3MS $\beta$-crystals. The WAXS profiles show that 5 and 11 $\mathrm{mol} \% 3 \mathrm{MS}$ contents gave $\beta$-dominated structure. Based on the heat of fusion and the crystallinity under different crystallization processes, the equilibrium heat of fusion of $\beta$-crystal $\left(\Delta \mathrm{H}_{\mathrm{m}}{ }^{0}\right)$ for sPS$3 \mathrm{MS}$ copolymers are: $88.0 \pm 2.6 \mathrm{MJ} \mathrm{m}^{-3}$ (sPS-5\%3MS) and $83.5 \pm 0.0 \mathrm{MJ} \mathrm{m}^{-3}$ (sPS-11\%3MS).

Presented in Figure S45 are the (a) DSC traces and (b) WAXS profiles of sPS-4MS $\alpha$ crystals. The WAXS profiles show that all specimens were all $\alpha$-dominated crystals, and, after peak deconvolution, the crystallinity $\left(X_{c}\right)$ can be calculated by the peak area of crystalline peaks over the total area. Combing the heat of fusion from DSC traces and the corresponding $X_{c}$ from WAXS profiles, the equilibrium heat of fusion of $\alpha$-crystals $\left(\Delta H_{m}{ }^{9}\right)$ can be estimated and the values are $91.8 \mathrm{MJ} \mathrm{m}^{-3}$ (sPS-2\%4MS), $105.4 \mathrm{MJ} \mathrm{m}^{-3}$ (sPS-5\%4MS) and $114.9 \mathrm{MJ} \mathrm{m}^{-3}$ (sPS-10\%4MS). 
(a) SPS-4MS DSC traces ( $\alpha$-crystal)

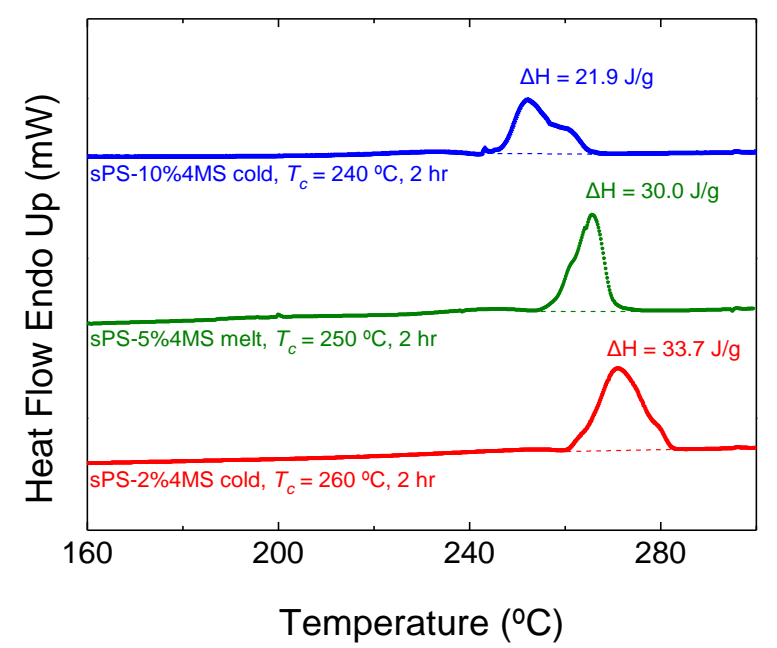

(b) SPS-4MS WAXS traces ( $\alpha$-crystal)

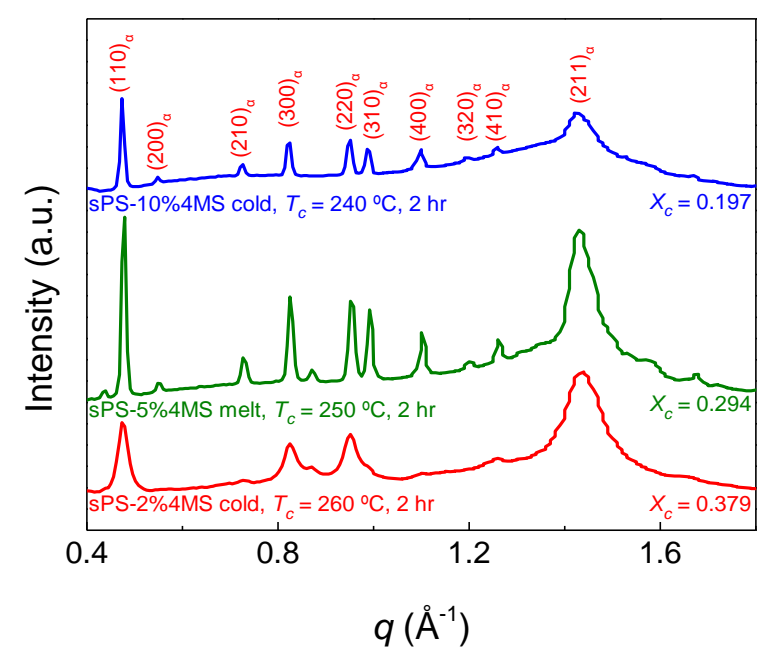

Figure S45. The (a) DSC heating traces (rate: $10{ }^{\circ} \mathrm{C} / \mathrm{min}$ ) and (b) WAXS profiles (collected at room temperature) for sPS-4MS $\alpha$-crystals. The WAXS profiles show that all specimens gave $\alpha$ dominated structure. Based on the heat of fusion and the crystallinity under different crystallization processes, the equilibrium heat of fusion of $\alpha$-crystal $\left(\Delta \mathrm{H}_{\mathrm{m}}{ }^{0}\right)$ for sPS-4MS copolymers are: $91.8 \mathrm{MJ}$ $\mathrm{m}^{-3}$ (sPS-2\%4MS), $105.4 \mathrm{MJ} \mathrm{m}^{-3}$ (sPS-5\%4MS) and $114.9 \mathrm{MJ} \mathrm{m}^{-3}$ (sPS-10\%4MS). (The systematic error is within 5\%)

Figure S46 presents the (a) DSC traces and (b) WAXS profiles of $\beta$-crystals of sPS-4MS with 2 mol\% 4MS content, which were prepared via melt-crystallization. The WAXS profiles show that both of the specimens were formed $\beta$-dominated crystals. Combing the heat of fusion from DSC traces and the corresponding $X_{c}$ from WAXS profiles, the equilibrium heat of fusion of $\beta$-crystals $\left(\Delta H_{m}{ }^{0}\right)$ of sPS- $2 \% 4 \mathrm{MS}$ is $129.1 \pm 1.2 \mathrm{MJ} \mathrm{m}^{-3}$.

(a) SPS-4MS DSC traces ( $\beta$-crystal)

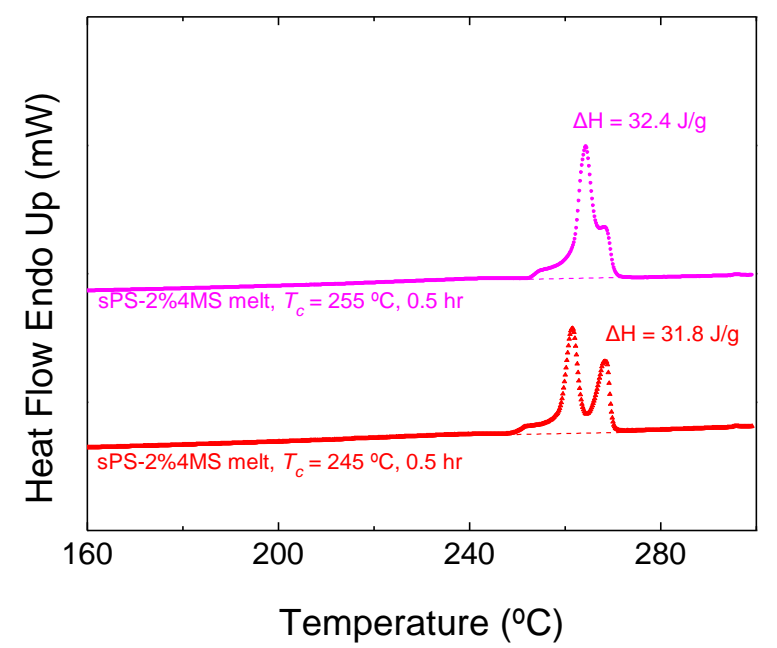

(b) SPS-4MS WAXS traces ( $\beta$-crystal)

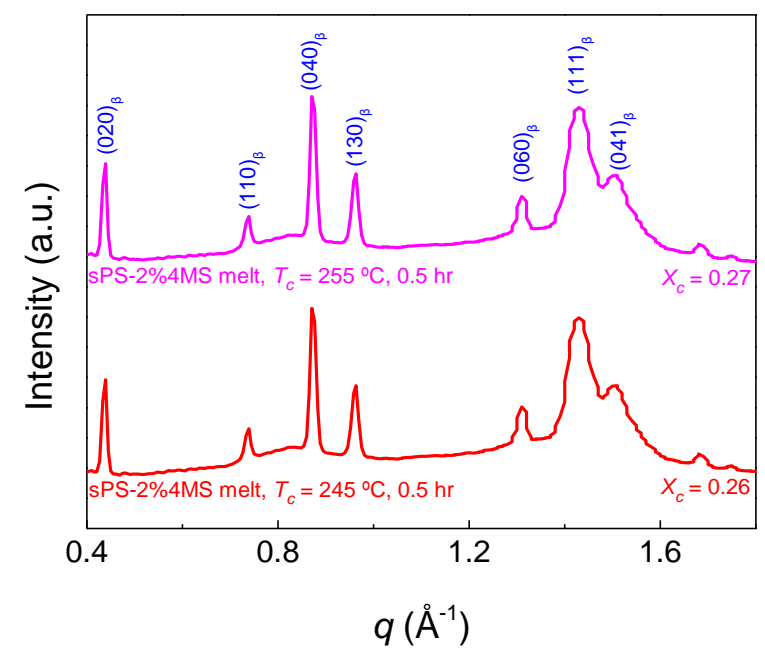

Figure S46. The (a) DSC heating traces (rate: $10^{\circ} \mathrm{C} / \mathrm{min}$ ) and (b) WAXS profiles (collected at room temperature) for melt-crystallized sPS-4MS $\beta$-crystals. Only sPS-4MS with 2 mol\% content gave $\beta$ dominated structure. Based on the heat of fusion and the crystallinity under different crystallization processes, the equilibrium heat of fusion of $\beta$-crystal $\left(\Delta \mathrm{H}_{m}{ }^{0}\right)$ for sPS-4MS copolymers are: $129.1 \pm$ $1.2 \mathrm{MJ} \mathrm{m}^{-3}$ (sPS-2\%4MS). 


\section{Evolution of Peak Position upon Heating}

Illustrated in Figure S47 are the evolutions of $(300)_{\alpha}$ and $(040)_{\beta}$ reflection peak positions upon heating in sPS-5\%3MS and SPS-2\%4MS systems. The light gray lines represent the linear dependence, which indicates the change of lattice parameters due to the thermal expansion, and the yellow background region indicates the higher temperature region that use to establish the equilibrium line in SAXS analyses. For sPS-5\%3MS, both $(300)_{\alpha}$ and $(040)_{\beta}$ (Figure S47a-b) reflections deviate from the linear relation and shift to lower- $q$ region at higher temperature region, representing a drastic lattice expansion while approaching melting. Crystals at higher temperatures have stronger ability to adjust the crystal structure to approach the solid-liquid equilibrium state, and these expansions suggest that the $\alpha$ - and $\beta$-phase could incorporate more comonomer units into the crystal during thickening at a higher temperature. For sPS-2\%4MS, the (300) $)_{\alpha}$ reflection also shows a sudden shift at higher temperatures (Figure S47c), but the shifting level is less than that of the sPS-5\%3MS case, which is due to the less comonomer content in sPS-2\%4MS system. The shift is even less significant for the $(040)_{\beta}$ in sPS-2\%4MS system (Figure S47d), but the result still suggests a weak tendency for lower- $q$ shifting phenomenon.
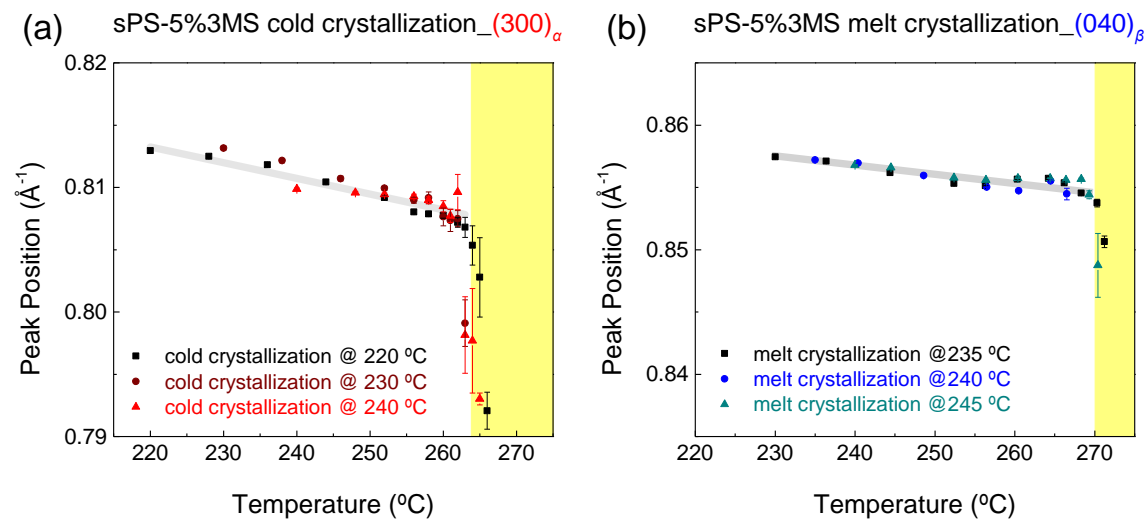

(c) sPS-2\%4MS cold crystallization_(300)
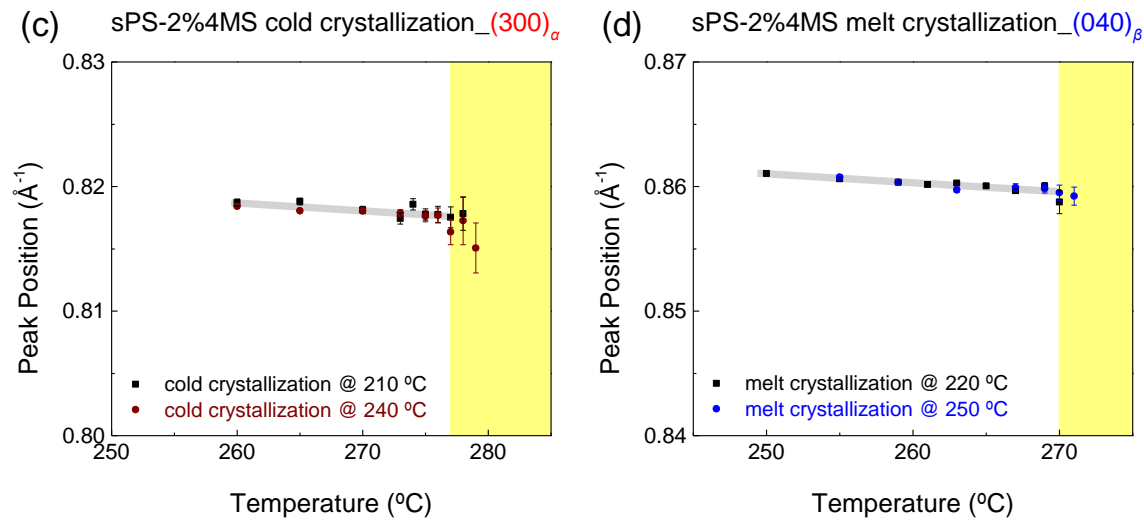

Figure S47. The evolution of $(300)_{\alpha}$ and $(040)_{\beta}$ reflection peak position upon heating for (a)-(b) sPS-5\%3MS and (c)-(d) sPS-2\%4MS systems. The reflection peak positions in (a)-(c) show a drastic lower- $q$ shifting phenomenon while approaching melting, and, although the lower- $q$ shifting is not apparent in $(040)_{\beta}$ of sPS-2\%4MS, the result still implies the tendency of lattice increase at higher temperatures. 


\section{$\underline{\alpha-a n d ~ \beta-s t r u c t u r e ~(M o l e c u l a r ~ M e c h a n i c s ~ C o m p u t a t i o n) ~}$}

The representative geometry optimized $\alpha$ - and $\beta$-structures of sPS-3MS and sPS-4MS random copolymers are illustrated in Figure S48, and the corresponding results are shown in our previous report ( $c f$. Figure 4 and the detailed description of methodology in reference 2 ). ${ }^{2}$ The methyl groups in $\alpha$ - or $\beta$-structure are all in the same styrene group but different positions. Figure S48 clearly shows that the $\alpha$-structure has higher compatibility for 4MS units, and introducing 3MS units causes stronger structure distortion; however, the situation is different for $\beta$-structure that the $\beta$ structure has higher compatibility for 3MS groups. Although the sPS-3MS $\alpha$-structure has the most significant deformation, the energy increase of the $\beta$-structure is still higher than the $\alpha$-structure due to the different packing energy. Thus, the $\alpha$-structure has higher tolerance for comonomer incorporation.

(a)

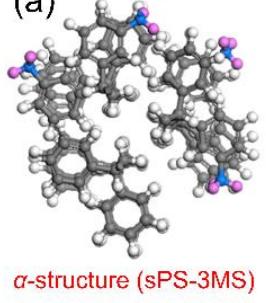

(b)

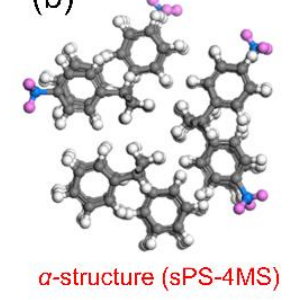

(c)

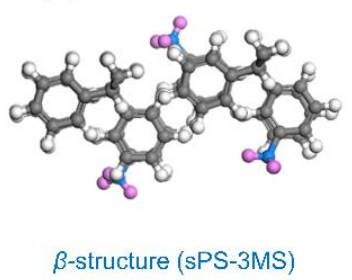

(d)

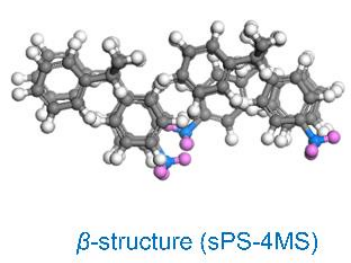

Figure S48. Representative (a)-(b) $\alpha$ - and (c)-(d) $\beta$-structures of sPS-3MS and sPS-4MS random copolymers that after geometry optimization with unit cell parameters fixed to the homopolymer values. The molecular mechanics computations shown that the $\alpha$-structure has higher tolerance for 4MS units and $\beta$-structure has higher tolerance for 3MS units. [C: gray atom, $\mathrm{H}$ : white atom, methyl unit: blue and pink] 


\section{$\underline{\alpha^{\prime \prime}-\text { form in } S P S-4 M S}$}

As described in our previous report ${ }^{2}$, the characteristic reflection peaks of $\alpha^{\prime \prime}$-phase emerge in the WAXS profiles of sPS-4MS, whereas the WAXS profiles of sPS-3MS correspond to the $\alpha^{\prime}-$ phase. The $\beta$-crystals formed in both sPS-3MS and sPS-4MS systems are all of the $\beta^{\prime}$-form. In the WAXS heating profiles of sPS-4MS $\alpha$-dominated structure ( $c f$. Figs S27, S31, and S35), the (310) $\alpha$ and $(400)_{\alpha}$ reflections, characteristic of the $\alpha^{\prime \prime}$-form, can be observed, which highlights the existence of $\alpha^{\prime \prime}$-structure and these reflections become more evident with increasing 4MS content. It should be noted that the $\alpha^{\prime \prime}$-form shares the same reflections of the $\alpha^{\prime}$-form but has additional peaks. Figure S49 shown the WAXS heating profiles of sPS-2\%4MS cold-crystallized specimen $\left(T_{c}=250{ }^{\circ} \mathrm{C}\right)$ at 200,260 , and $274{ }^{\circ} \mathrm{C}$, and the dash lines represent the position of $(310)_{\alpha}$ and $(400)_{\alpha}$ reflections. These two reflections become obvious with increasing temperature, suggesting the generation of $\alpha$ "-form during the heating scan. However, the (220) $\alpha$ reflection become sharper at higher temperatures; the values of the corresponding full-width-at-half-maximum (FWHM) at different temperature are summarized in Table S1. Thus, the possibility that these $\alpha$ "-form reflections are initially overlapped with the stronger reflection (ex. (220) $)$ ) cannot be excluded. The corresponding populations of $\alpha^{\prime}$ - and $\alpha^{\prime \prime}$-form in sPS-4MS systems cannot be accurately determined herein; more careful experimental design is needed to distinguish these two sub-modifications.

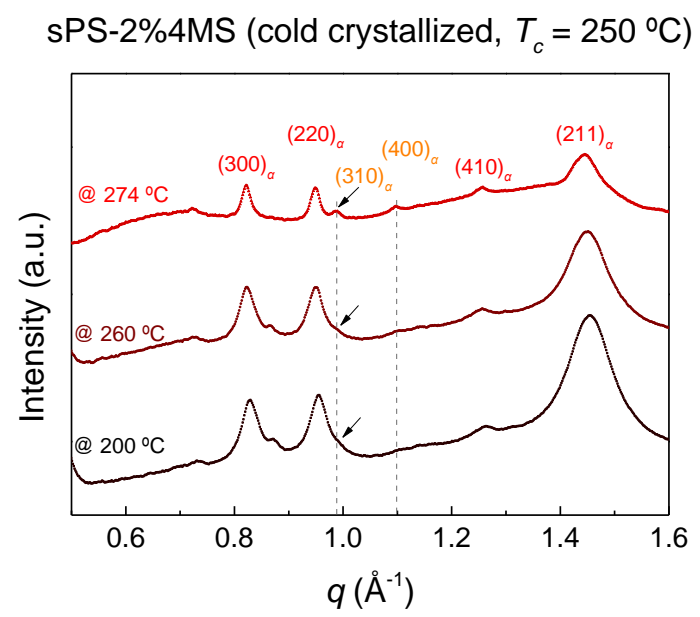

Figure S49. Selected WAXS heating profiles of sPS-2\%4MS cold-crystallized specimen $\left(T_{c}=250\right.$ ${ }^{\circ} \mathrm{C}, c f$. Figure S27f). The $(310)_{\alpha}$ and $(400)_{\alpha}$ reflections are characteristic of the $\alpha^{\prime \prime}$-structure; the rest reflections are shared with the $\alpha^{\prime}$-structure.

Table S1. The value of full-width-at-half-maximum (FWHM) of (220) $\alpha_{\alpha}$ reflection upon heating, showing that the $(220)_{\alpha}$ reflection become sharper at elevated temperature.

\begin{tabular}{|l|l|l|l|}
\hline Temperature & $200{ }^{\circ} \mathrm{C}$ & $260{ }^{\circ} \mathrm{C}$ & $274{ }^{\circ} \mathrm{C}$ \\
\hline FWHM of $(220)_{\alpha}$ & $0.04395 \AA^{-1}$ & $0.03585 \AA^{-1}$ & $0.01755 \AA^{-1}$ \\
\hline
\end{tabular}




\section{$\underline{\text { Reference }}$}

(1) Su, C. H.; Jeng, U.; Chen, S. H.; Cheng, C. Y.; Lee, J. J.; Lai, Y. H.; Su, W. C.; Tsai, J. C.; $\mathrm{Su}, \mathrm{A}$. C. Thermodynamic Characterization of Polymorphs in Bulk-Crystallized Syndiotactic Polystyrene via Small/Wide-Angle X-ray Scattering and Differential Scanning Calorimetry. Macromolecules 2009, 42, 4200-4207.

(2) Chen, P.-H.; Lan, Y.-K.; Lin, S.-J.; Tsai, J.-C.; Jeng, U. S.; Su, A.-C. Crystallization of $\alpha$ versus $\beta$ Phases in Syndiotactic Poly(styrene-stat-3-methylstyrene) and Poly(styrene-stat-4methylstyrene). ACS Appl. Polym. Mater. 2019, 1, 251-258. 